Supporting Information for:

\title{
Norbornadienes: Robust and Scalable Building Blocks for Cascade "Click" Coupling of High Molecular Weight Polymers
}

Andre H. St. Amant, ${ }^{\dagger \S}$ Emre H. Discekici, ${ }^{\dagger \S}$ Sophia J. Bailey,${ }^{\dagger}$ Manuel S. Zayas, ${ }^{\dagger}$ Jung-Ah, Song, ${ }^{\ddagger}$ Shelby L. Shankel, ${ }^{\dagger}$ Shay N. Nguyen, ${ }^{\dagger}$ Morgan W. Bates, ${ }^{\ddagger}$ Athina Anastasaki,${ }^{\ddagger}$ Craig J. Hawker, ${ }^{*+\perp}$ and Javier Read de Alaniz ${ }^{* \dagger}$

${ }^{\dagger}$ Department of Chemistry and Biochemistry, Materials Research Laboratory, and ${ }^{\perp}$ Department of Materials, University of California, Santa Barbara, California 93106, United States

Table of Contents

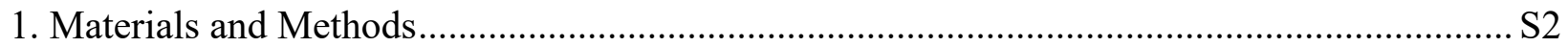

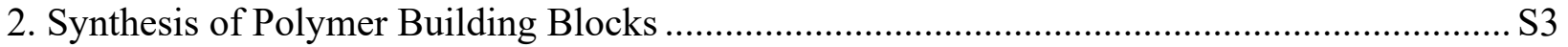

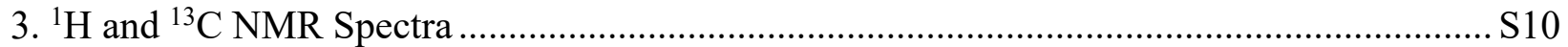

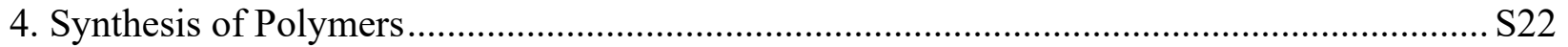

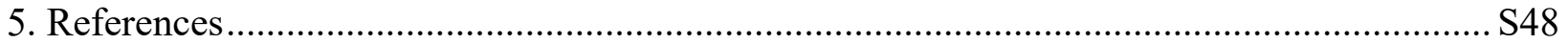




\section{Materials and Methods}

Unless stated otherwise, reactions were conducted under an atmosphere of $\mathrm{N}_{2}$ using reagent grade solvents. Dichloromethane (DCM) and methanol (MeOH) were stored over $3 \AA$ molecular sieves. Tetrahydrofuran (THF) was passed over a column of activated alumina. All commercially obtained reagents were used as received. All commercial vinyl monomers were purified by passing through a plug of basic alumina unless otherwise noted. Thin-layer chromatography (TLC) was conducted with E. Merck silica gel 60 F254 pre-coated plates $(0.25 \mathrm{~mm})$ and visualized by exposure to UV light $(254 \mathrm{~nm})$ or stained with $p$-anisaldehyde, potassium permanganate, or bromocresol green. Flash column chromatography was performed using normal phase silica gel (60 $\AA, 0.040-0.063$ $\mathrm{mm}$, Geduran). ${ }^{1} \mathrm{H}$ NMR spectra were recorded on Varian spectrometers $(400,500$, or $600 \mathrm{MHz})$ and are reported relative to the residual protio-signal in deuterated solvents. Data for ${ }^{1} \mathrm{H}$ NMR spectra are reported as follows: chemical shift $(\delta \mathrm{ppm})$, multiplicity, coupling constant $(\mathrm{Hz})$ and integration. ${ }^{13} \mathrm{C}$ NMR spectra were recorded on Varian Spectrometers $(100,125$, or $150 \mathrm{MHz})$. Data for ${ }^{13} \mathrm{C}$ NMR spectra are reported in terms of chemical shift $(\delta \mathrm{ppm})$. Mass spectra were obtained from the UC Santa Barbara Mass Spectrometry Facility on a (Waters Corp.) GCT Premier high resolution Time-of-flight mass spectrometer with an ESI or EI source. Matrix-Assisted Laser Desorption Ionization time of flight mass spectrometry (MALDI-ToF-MS) was conducted using a Bruker Microflex LRF MALDI TOF mass spectrometer equipped with a $60 \mathrm{~Hz}$ nitrogen laser at $337 \mathrm{~nm}$. Solutions in THF of dithranol as a matrix (saturated solution, $10 \mu \mathrm{L}$ ), sodium bromide as cationization agent $(1.0 \mathrm{mg} / \mathrm{ml}, 2 \mu \mathrm{L})$ were mixed with the sample $(1.0 \mathrm{mg} / \mathrm{ml}, 10 \mu \mathrm{L})$ and $1.0 \mu \mathrm{L}$ of the mixture was applied to the target plate. Size exclusion chromatography (SEC) eluting with chloroform $\left(\mathrm{CHCl}_{3}\right)$ or THF and calibrated relative to polystyrene standards was performed on a Waters Alliance HPLC System, 2695 separation module with Wyatt DAWN HELEOS-II light scattering and Wyatt Optilab rEX refractive index detectors and/or a Waters Acquity Advanced Polymer Characterization (APC) System equipped with an Acquity UPLC refractive index detector. Synchrotron small-angle X-ray scattering (SAXS) measurements were performed at sector 5-ID-D of the Advanced Photon Source (Argonne, IL). Experiments employed a sampleto-detector distance of $8505 \mathrm{~mm}$ calibrated using a silver behenate standard. 2D scattering patterns were recorded on a Rayonix CCD area detector using typical exposure times of 1-3 s. The isotropic $2 \mathrm{D}$ patterns were azimuthally integrated to the one-dimensional form of intensity as a function of the magnitude of the scattering wave vector $q=|\mathbf{q}|=4 \pi \sin (\theta / 2) / \lambda$, where $\lambda$ is the nominal x-ray wavelength $(\lambda=0.729 \AA)$ and $\theta$ is the scattering angle. 


\section{Synthesis of Polymer Building Blocks}

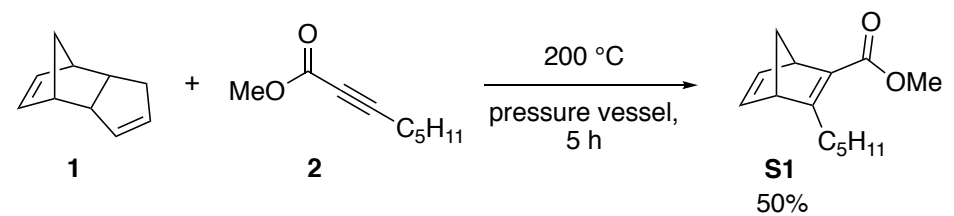

Methyl 3-pentylbicyclo[2.2.1]hepta-2,5-diene-2-carboxylate (S1):

Methyl 2-octynoate (6.7 mL, $40 \mathrm{mmol}, 1.0$ eq.), dicyclopentadiene (3.2 mL, $24 \mathrm{mmol}, 1.2$ eq. of cyclopentadiene), and hydroquinone (100 $\mathrm{mg}$ ) were added to a pressure vessel and sealed under an atmosphere of $\mathrm{N}_{2}$. The vessel was heated to $200{ }^{\circ} \mathrm{C}$ for $5 \mathrm{~h}(63 \%$ conversion by NMR). The crude product was distilled twice $\left(100-120^{\circ} \mathrm{C}, \sim 200 \mathrm{mTorr}\right)$ to afford racemic S1 (4.4 g, $20 \mathrm{mmol}$, $50 \%$ ) as a clear and colorless liquid.

\section{Large-scale modification:}

Methyl 2-octynoate (59.0 mL, $350 \mathrm{mmol}, 1$ eq.), dicyclopentadiene (28.3 mL, $210 \mathrm{mmol}, 0.6$ eq.), and hydroquinone $(0.87 \mathrm{~g})$ were added to a $150 \mathrm{~mL}$ pressure vessel and sealed under an atmosphere of argon. The vessel was heated in a silicone oil bath to $200^{\circ} \mathrm{C}$ for $6 \mathrm{~h}$ ( $84 \%$ conversion by NMR). The crude product was distilled once $\left(110-115{ }^{\circ} \mathrm{C}, \sim 275 \mathrm{mTorr}\right)$ to afford racemic $\mathbf{S 1}$ as a clear and colorless liquid (40.8 g, $185 \mathrm{mmol}, 53 \%$ ).

S1: ${ }^{1} \mathrm{H}$ NMR $\left(400 \mathrm{MHz}, \mathrm{CDCl}_{3}\right) \delta 6.88(\mathrm{dd}, J=3.1,4.7 \mathrm{~Hz}, 1 \mathrm{H}), 6.70(\mathrm{dd}, J=3.3,4.5 \mathrm{~Hz}, 1 \mathrm{H})$, 3.88 (br. s., 1 H), 3.72 (s, 3 H), 3.51 (br. s., 1 H), 2.75 - 2.60 (m, 2 H), 2.00 (tdd, J = 1.6, 6.3, 25.4 $\mathrm{Hz}, 2 \mathrm{H}), 1.55-1.21(\mathrm{~m}, 6 \mathrm{H}), 0.89(\mathrm{t}, J=7.0 \mathrm{~Hz}, 3 \mathrm{H}) \mathrm{ppm} ;{ }^{13} \mathrm{C} \mathrm{NMR}\left(100 \mathrm{MHz}, \mathrm{CDCl}_{3}\right) \delta 173.9$, 166.3, 143.7, 140.7, 138.0, 71.0, 56.0, 51.0, 50.8, 31.5, 30.2, 26.4, 22.4, 13.9 ppm; IR (ATR) 2957, 2931, 2863, 1705, 1625, 1433, 1338, 1294, 1235, 1100, 1079, $714 \mathrm{~cm}^{-1}$; HRMS (EI+) Exact mass cald. for $\mathrm{C}_{14} \mathrm{H}_{20} \mathrm{O}_{2}^{+}[\mathrm{M}]^{+}: 220.1458$, found: 220.1466 .
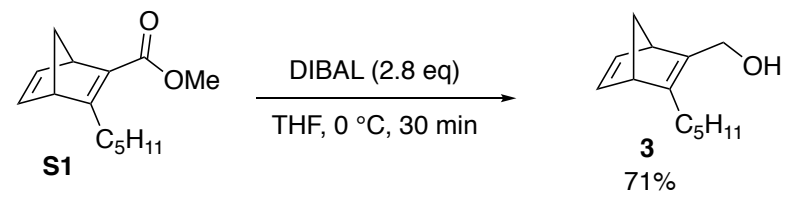

(3-Pentylbicyclo[2.2.1]hepta-2,5-dien-2-yl)methanol (3):

A solution of $\mathbf{S 1}(1.76 \mathrm{~g}, 8.01 \mathrm{mmol}, 1.0$ eq. $)$ in THF $(100 \mathrm{~mL})$ was cooled to $0{ }^{\circ} \mathrm{C}$. A solution of diisobutylaluminium hydride (DIBAL) in hexanes ( $1 \mathrm{M}, 22.4 \mathrm{~mL}, 22.4 \mathrm{mmol}, 2.8$ eq.) was added dropwise over $10 \mathrm{~min}$. The solution was maintained at $0{ }^{\circ} \mathrm{C}$ until complete consumption of the starting material (TLC, $30 \mathrm{~min}$ ). The reaction was carefully quenched at $0{ }^{\circ} \mathrm{C}$ sequentially with water $(0.8 \mathrm{~mL})$, an aqueous solution of sodium hydroxide $(\mathrm{NaOH})(1 \mathrm{M}, 1.6 \mathrm{~mL})$, and water $(0.8$ $\mathrm{mL}$ ). The reaction mixture was removed from the ice bath and stirred a further $30 \mathrm{~min}$. The reaction mixture was dried with $\mathrm{MgSO}_{4}$, stirred a further $10 \mathrm{~min}$, then filtered and rinsed with diethyl ether $\left(\mathrm{Et}_{2} \mathrm{O}\right)(150 \mathrm{~mL})$. The solvent was removed and the residue subjected to flash column chromatography hexanes and ethyl acetate (EtOAc) as eluent (hexanes:EtOAc, 6:1) to yield racemic $3(1.09 \mathrm{~g}, 5.67 \mathrm{mmol}, 71 \%)$ as a clear and colorless oil. Spectral data matched that of literature reported data. ${ }^{1}$ 


\section{Large-scale modification:}

A $3 \mathrm{~L}$ three-neck round bottom flask equipped with stir bar, drop funnel and internal temperature probe was charged with THF (1.2 L) and $\mathbf{S 1}$ ( $40.8 \mathrm{~g}, 185 \mathrm{mmol}, 1.0 \mathrm{eq}$.). The solution was cooled to $0{ }^{\circ} \mathrm{C}$ and a solution of DIBAL in hexanes $(1 \mathrm{M}, 518 \mathrm{~mL}, 518 \mathrm{mmol}, 2.8$ eq.) was added via drop funnel over $1.25 \mathrm{~h}$ while keeping the internal flask temperature between 0 and $5{ }^{\circ} \mathrm{C}$. The solution was maintained at $0{ }^{\circ} \mathrm{C}$ until complete consumption of the starting material (TLC, $10 \mathrm{~min}$ ). The reaction was carefully quenched at $0{ }^{\circ} \mathrm{C}$ with water $(19 \mathrm{~mL})$ over $30 \mathrm{~min}$, an aqueous solution of $\mathrm{NaOH}(1 \mathrm{M}, 37.5 \mathrm{~mL})$ over $10 \mathrm{~min}$, and water $(19 \mathrm{~mL})$ over $10 \mathrm{~min}$. The reaction mixture was removed from the ice bath, stirred an additional $1 \mathrm{~h}$ and dried with $\mathrm{MgSO}_{4}(100 \mathrm{~g})$. The slurry was stirred a further $10 \mathrm{~min}$, then filtered and rinsed with $\mathrm{Et}_{2} \mathrm{O}(750 \mathrm{~mL})$. The solvent was removed and the residue subjected to a silica plug (hexanes:EtOAc, 1:1) to yield racemic 3 (21.7 g, 113 mmol, 61\%) as a clear and light yellow oil.

3: $\mathrm{R}_{f}$ (hexanes:EtOAc, 6:1): 0.29; ${ }^{1} \mathrm{H}$ NMR $\left(400 \mathrm{MHz}, \mathrm{CDCl}_{3}\right) \delta 6.81(\mathrm{dd}, J=2.7,5.1 \mathrm{~Hz}, 1 \mathrm{H})$, $6.73(\mathrm{dd}, J=3.1,5.1 \mathrm{~Hz}, 1 \mathrm{H}), 4.26(\mathrm{dd}, J=5.5,12.1 \mathrm{~Hz}, 1 \mathrm{H}), 4.14(\mathrm{dd}, J=4.7,12.1 \mathrm{~Hz}, 1 \mathrm{H})$, 3.55 (br. s., 1 H), 3.38 (br. s., 1 H), 2.27 - 2.17 (m, 1 H), 2.17 - 2.07 (m, 1 H), 1.92 (dd, $J=5.9$, $15.7 \mathrm{~Hz}, 2 \mathrm{H}), 1.47-1.24(\mathrm{~m}, 4 \mathrm{H}), 1.24-1.12(\mathrm{~m}, 2 \mathrm{H}), 0.98(\mathrm{t}, J=5.5 \mathrm{~Hz}, 1 \mathrm{H}), 0.88(\mathrm{t}, J=$ $7.2 \mathrm{~Hz}, 3 \mathrm{H}) \mathrm{ppm} ;{ }^{13} \mathrm{C} \mathrm{NMR}\left(100 \mathrm{MHz}, \mathrm{CDCl}_{3}\right) \delta 152.9,145.1,143.0,142.2,71.4,58.9,53.7$, 51.8, 31.4, 28.2, 27.0, 22.5, 14.0 ppm; IR (ATR) 3324, 2959, 2928, 2860, 1457, 1378, 1304, 1230, 1209, 1016, $987 \mathrm{~cm}^{-1}$; HRMS (EI+) Exact mass cald. for $\mathrm{C}_{13} \mathrm{H}_{18}{ }^{+}\left[\mathrm{M}-\mathrm{H}_{2} \mathrm{O}\right]^{+}:$174.1403, found: 174.1408 .

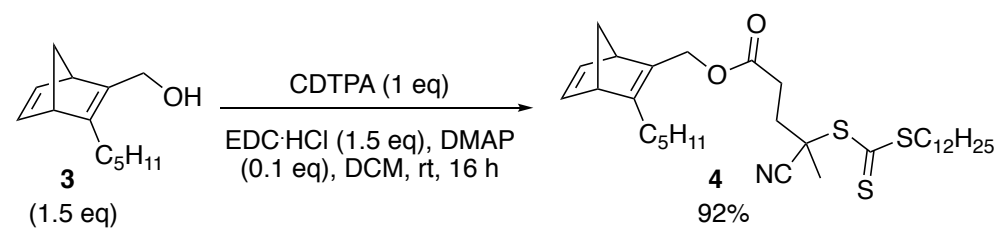

\section{(3-pentylbicyclo[2.2.1]hepta-2,5-dien-2-yl)methyl 4-cyano-4- (((dodecylthio)carbonothioyl)thio)pentanoate (4):}

Compound 3 (144 mg, $0.75 \mathrm{mmol}, 1.5$ eq.), $\mathrm{EDC} \cdot \mathrm{HCl}(14 \mathrm{mg}, 0.75 \mathrm{mmol}, 1.5 \mathrm{eq}$.$) , and$ dimethylaminopyridine (DMAP, $6 \mathrm{mg}, 0.050 \mathrm{mmol}, 0.10$ eq.) were added to a solution of 4-cyano4-[(dodecylsulfanylthiocarbonyl)sulfanyl]pentanoic acid (202 mg, $0.50 \mathrm{mmol}, 1.0$ eq.) in DCM (1 $\mathrm{mL}$ ) in a vial. The reaction was capped under an atmosphere of air and stirred at $\mathrm{rt}$ for $16 \mathrm{~h}$ (the starting material was consumed by NMR). The reaction mixture was adsorbed to silica gel ( $2 \mathrm{~g}$ ) and subjected to flash column chromatography (hexanes:EtOAc, 15:1) to yield diastereomeric 4 (266 mg, $0.46 \mathrm{mmol}, 92 \%$ ) as a yellow oil.

4: $\mathrm{R}_{f}$ (Hexanes:EtOAc, 15:1): 0.21; ${ }^{1} \mathrm{H}$ NMR (400 MHz, $\left.\mathrm{CDCl}_{3}\right) \delta 6.77(\mathrm{dd}, J=3.0,5.1 \mathrm{~Hz}, 1 \mathrm{H}$ ), $6.72(\mathrm{dd}, J=2.9,4.7 \mathrm{~Hz}, 1 \mathrm{H}), 4.75(\mathrm{dd}, J=1.0,12.2 \mathrm{~Hz}, 1 \mathrm{H}), 4.61(\mathrm{~d}, J=11.9 \mathrm{~Hz}, 1 \mathrm{H}), 3.45$ (br. s., $1 \mathrm{H}), 3.39$ (br. s., $1 \mathrm{H}), 3.34$ (t, J=7.4 Hz, $2 \mathrm{H}), 2.68$ - 2.57 (m, $2 \mathrm{H}), 2.57$ - 2.48 (m, $1 \mathrm{H})$, $2.42-2.34(\mathrm{~m}, 1 \mathrm{H}), 2.24(\mathrm{ddd}, J=6.5,8.0,14.1 \mathrm{~Hz}, 1 \mathrm{H}), 2.15(\mathrm{ddd}, J=6.5,8.0,14.1 \mathrm{~Hz}, 1 \mathrm{H})$, $1.96(\mathrm{td}, J=1.6,6.0 \mathrm{~Hz}, 1 \mathrm{H}), 1.91(\mathrm{td}, J=1.6,6.0 \mathrm{~Hz}, 1 \mathrm{H}), 1.88(\mathrm{~s}, 3 \mathrm{H}), 1.70$ (quin, $J=7.5 \mathrm{~Hz}$, $2 \mathrm{H}), 1.45-1.14(\mathrm{~m}, 24 \mathrm{H}), 0.89(\mathrm{dt}, J=3.1,7.1 \mathrm{~Hz}, 6 \mathrm{H}) \mathrm{ppm} ;{ }^{13} \mathrm{C} \mathrm{NMR}\left(125 \mathrm{MHz}, \mathrm{CDCl}_{3}\right) \delta$ 
216.9, 171.5, 156.1, 142.8, 142.0, 140.6, 119.0, 71.4, 61.3, 53.8, 52.2, 46.4, 37.0, 33.9, 31.9, 31.4, 29.8, 29.6, 29.5, 29.4, 29.3, 29.0, 28.9, 28.4, 27.7, 26.8, 24.8, 22.7, 22.5, 14.1, 14.0 ppm; IR (ATR) 2954, 2923, 2853, 1737, 1645, 1456, 1378, 1291, 1176, 1068, 861, $806 \mathrm{~cm}^{-1}$; HRMS (ES+) Exact mass cald. for $\mathrm{C}_{32} \mathrm{H}_{51} \mathrm{NNaO}_{2} \mathrm{~S}_{3}{ }^{+}[\mathrm{M}+\mathrm{Na}]^{+}$: 600.2974 , found: 600.3001 .

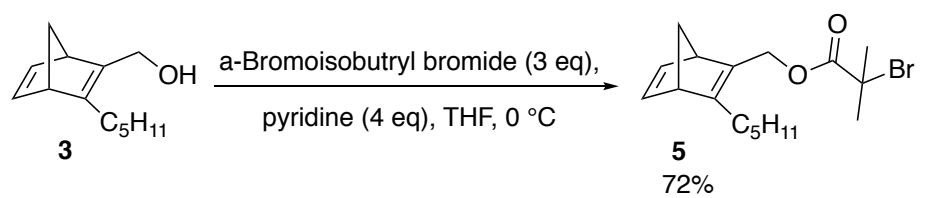

3-pentylbicyclo[2.2.1] hepta-2,5-dien-2-yl)methyl 2-bromo-2-methylpropanoate (5):

Compound 3 (0.380 g, $1.98 \mathrm{mmol}, 1.0$ eq.) and pyridine (0.64 mL, $7.90 \mathrm{mmol}, 4.0$ eq.) were added to THF $(13 \mathrm{~mL})$ and cooled to $0{ }^{\circ} \mathrm{C}$. $\alpha$-Bromoisobutyryl bromide $(0.37 \mathrm{~mL}, 2.96 \mathrm{mmol}, 1.5$ eq. $)$, was added drop-wise and the reaction solution was stirred at $0{ }^{\circ} \mathrm{C}$ for $2 \mathrm{~h}$. The solvent removed and the residue was subjected to flash column chromatography (Hexanes:EtOAc, 20:1 and 2\% triethylamine $\left.\left(\mathrm{Et}_{3} \mathrm{~N}\right)\right)$ to yield $5(0.485 \mathrm{~g}, 1.42 \mathrm{mmol}, 72 \%)$ as a clear and colorless oil. The purified product was kept in a solution of hexane/EtOAc/Et $3 \mathrm{~N}$ for stability.

$\mathrm{R}_{f}$ (Hexanes:EtOAc, 10:1) 0.35; ${ }^{1} \mathrm{H}$ NMR $\left(600 \mathrm{MHz}, \mathrm{CDCl}_{3}\right) \delta 6.75(\mathrm{~m}, 1 \mathrm{H}), 6.69(\mathrm{~m}, 1 \mathrm{H}), 4.81$ $(\mathrm{d}, 1 \mathrm{H}), 4.66(\mathrm{~d}, 1 \mathrm{H}), 3.48(\mathrm{t}, 1 \mathrm{H}), 3.36(\mathrm{t}, 1 \mathrm{H}), 2.23(\mathrm{~m}, 1 \mathrm{H}), 2.18(\mathrm{~m}, 1 \mathrm{H}), 1.94(\mathrm{~m}, 1 \mathrm{H}), 1.91(\mathrm{~s}$, $6 \mathrm{H}), 1.89(\mathrm{~m}, 1 \mathrm{H}), 1.38(\mathrm{~m}, 2 \mathrm{H}), 1.27(\mathrm{~m}, 2 \mathrm{H}), 1.17(\mathrm{~m}, 2 \mathrm{H}), 0.85(\mathrm{t}, 3 \mathrm{H}) \mathrm{ppm} .{ }^{13} \mathrm{C}$ NMR $(126$ $\left.\mathrm{MHz}, \mathrm{CDCl}_{3}\right) \delta 171.80,156.44,143.15,141.99,140.68,71.60,62.32,56.29,54.02,52.32,31.52$, 31.03, 28.57, 26.98, 22.65, 14.20 ppm. IR (ATR): 2937, 2929, 2860, 1732, 1462, 1270, 1159, 1107 $\mathrm{cm}^{-1}$; HRMS (ES+) Exact mass cald. for $\mathrm{C}_{17} \mathrm{H}_{25} \mathrm{BrO}_{2} \mathrm{Na}^{+}[\mathrm{M}+\mathrm{Na}]^{+}: 363.1$, found: 363.1 .

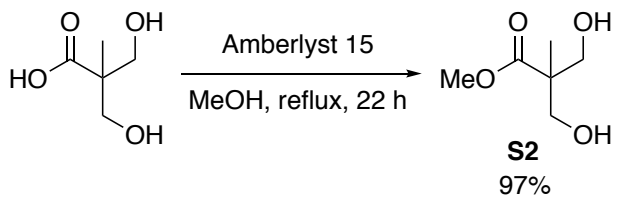

Methyl 3-hydroxy-2-(hydroxymethyl)-2-methylpropanoate (S2): ${ }^{2}$

2,2-Bis(hydroxymethyl)propionic acid (10.0 g, $74.6 \mathrm{mmol}, 1 \mathrm{eq}$.$) and Amberlyst 15$ hydrogen form $(3.50 \mathrm{~g})$ were added to $\mathrm{MeOH}(75 \mathrm{~mL})$ and the mixture was heated to reflux for $22 \mathrm{~h}$ (the reaction was complete by NMR). The reaction was cooled to rt, filtered through Celite, rinsed with $\mathrm{MeOH}(25 \mathrm{~mL})$, and the solvent removed. The residue was dissolved in DCM $(25 \mathrm{~mL})$, filtered through Celite, and the solvent removed to yield S2 (10.7 g, $72.2 \mathrm{mmol}, 97 \%)$ as an amber oil that solidified on standing. The purity was sufficient for the next reaction. Spectral data matched that of the reported literature. ${ }^{2}$

S2: ${ }^{1} \mathrm{H}$ NMR (600 MHz, $\left.\mathrm{CDCl}_{3}\right) \delta 3.90(\mathrm{~d}, \mathrm{~J}=11.1 \mathrm{~Hz}, 2 \mathrm{H}), 3.76(\mathrm{~s}, 3 \mathrm{H}), 3.71(\mathrm{~d}, \mathrm{~J}=11.5 \mathrm{~Hz}$, $2 \mathrm{H}), 3.00$ (br. s., $2 \mathrm{H}), 1.07$ (s, $3 \mathrm{H}$ ) ppm. 


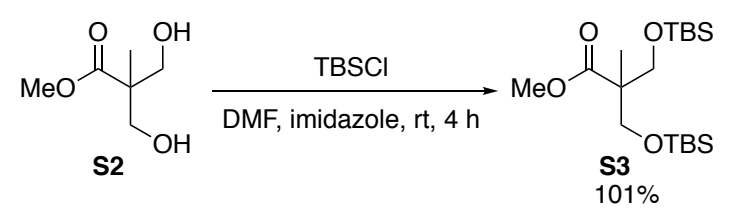

Methyl 3-((tert-butyldimethylsilyl)oxy)-2-(((tert-butyldimethylsilyl)oxy)methyl)-2methylpropanoate (S3):

tert-Butyldimethylsilyl chloride ( $\mathrm{TBSCl}, 5.04 \mathrm{~g}, 33.4 \mathrm{mmol}, 2.2 \mathrm{eq}$.) was added in portions to a solution of S2 (2.25 g, $15.2 \mathrm{mmol}, 1.0$ eq.) and imidazole (2.48 g, $36.5 \mathrm{mmol}, 2.4$ eq.) in dimethylformamide (DMF, $20 \mathrm{~mL}$ ). After $2 \mathrm{~h}$ the reaction did not go to completion (NMR) and a further imidazole $(0.414 \mathrm{~g}, 6.08 \mathrm{mmol}, 0.40$ eq.) and TBSCl $(0.916 \mathrm{~g}, 6.08 \mathrm{mmol}, 0.4$ eq.) was added. After $2 \mathrm{~h}$, the reaction was deemed complete (NMR). The reaction was poured into an aqueous solution of $\mathrm{HCl}(1 \mathrm{M}, 100 \mathrm{~mL})$ and extracted with $\mathrm{Et}_{2} \mathrm{O}(2 \times 100 \mathrm{~mL})$. The combined organic layers were washed with brine $(2 \times 50 \mathrm{~mL})$, dried over $\mathrm{MgSO}_{4}$, and the solvent removed to yield S3 (5.80 g, $15.4 \mathrm{mmol}, 101 \%)$ as a clear and colorless oil that was used directly in the next reaction.

S3: ${ }^{1} \mathrm{H}$ NMR (400 MHz, $\left.\mathrm{CDCl}_{3}\right) \delta 3.73(\mathrm{~d}, \mathrm{~J}=9.4 \mathrm{~Hz}, 2 \mathrm{H}), 3.69$ - $3.61(\mathrm{~m}, 5 \mathrm{H}), 1.10(\mathrm{~s}, 3 \mathrm{H})$, $0.87(\mathrm{~s}, 18 \mathrm{H}), 0.03(\mathrm{~s}, 12 \mathrm{H}) \mathrm{ppm} ;{ }^{13} \mathrm{C} \mathrm{NMR}\left(100 \mathrm{MHz}, \mathrm{CDCl}_{3}\right) \delta$ 175.6, 64.2, 51.3, 50.3, 25.8, $18.2,17.0,-5.61,-5.64 \mathrm{ppm}$.

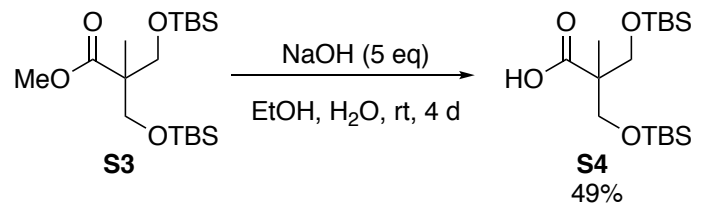

3-((tert-Butyldimethylsilyl)oxy)-2-(((tert-butyldimethylsilyl)oxy)methyl)-2methylpropanoic acid (S4):

S3 (1.88 g, $5 \mathrm{mmol}, 1$ eq.) was dissolved in ethanol (EtOH,50 mL). An aqueous solution of $\mathrm{NaOH}$ (1 M, $25 \mathrm{~mL}, 25 \mathrm{mmol}, 5$ eq.) was added, and the biphasic mixture was stirred vigorously for 4 days. The reaction mixture, then homogeneous, was poured into an aqueous solution of $\mathrm{HCl}(1 \mathrm{M}$, $50 \mathrm{~mL})$ and extracted with EtOAc $(2 \times 100 \mathrm{~mL})$. The combined organic layers were washed with brine $(50 \mathrm{~mL})$, dried over $\mathrm{MgSO}_{4}$, and the solvent removed. The residue was subjected to flash column chromatography (hexanes:EtOAc, 9:1) to yield $\mathbf{S 4}$ (897 $\mathrm{mg}, 2.47 \mathrm{mmol}, 49 \%)$ as a white solid. Spectral data matched that of the reported literature. ${ }^{3}$

S4: $\mathrm{R}_{f}$ (Hexanes:EtOAc, 9:1): 0.28; ${ }^{1} \mathrm{H}$ NMR (400 MHz, $\mathrm{CDCl}_{3}$ ) $\delta 11.12$ (br. s., $1 \mathrm{H}$ ), 3.75 (d, J = $9.8 \mathrm{~Hz}, 2 \mathrm{H}), 3.70$ (d, J = 9.4 Hz, $2 \mathrm{H}), 1.15$ (s, $3 \mathrm{H}), 0.90$ (s, $18 \mathrm{H}), 0.07$ (s, $12 \mathrm{H}) \mathrm{ppm} ;{ }^{13} \mathrm{C}$ NMR $\left(100 \mathrm{MHz}, \mathrm{CDCl}_{3}\right) \delta 178.4,64.5,49.4,25.8,18.2,17.1,-5.6 \mathrm{ppm}$. 


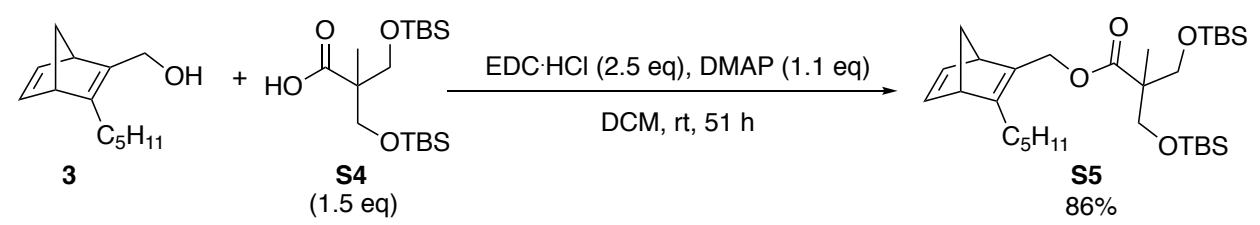

(3-pentylbicyclo[2.2.1] hepta-2,5-dien-2-yl)methyl 3-((tert-butyldimethylsilyl)oxy)-2-(((tertbutyldimethylsilyl)oxy)methyl)-2-methylpropanoate (S5):

$N$-(3-Dimethylaminopropyl)- $N$ '-ethylcarbodiimide hydrochloride (EDC $\cdot \mathrm{HCl}, 288 \mathrm{mg}, 1.5 \mathrm{mmol}$, 1.5 eq.), and DMAP (12 mg, $0.1 \mathrm{mmol}, 0.1$ eq.) were added to a solution of $3(192 \mathrm{mg}, 1.0 \mathrm{mmol}$, 1.0 eq.) and $\mathbf{S 4}(544 \mathrm{mg}, 1.5 \mathrm{mmol}, 1.5$ eq. $)$ in DCM $(1 \mathrm{~mL})$ in a vial. The reaction was capped under an atmosphere of air and stirred $23 \mathrm{~h}$ at $\mathrm{rt}$. The conversion was low ( 18\% by NMR). Additional DMAP (110 mg, $0.9 \mathrm{mmol}, 0.9$ eq.) was added, and the reaction mixture stirred $20 \mathrm{~h}$. Addtional $\mathrm{EDC} \cdot \mathrm{HCl}(192 \mathrm{mg}, 1.0 \mathrm{mmol}, 1.0 \mathrm{eq}$.) was added, the reaction mixture stirred for $8 \mathrm{~h}$, and the reaction was deemed complete (NMR). The mixture was adsorbed to silica gel (1.5 g) and subjected to flash column chromatography (hexanes:EtOAc, 1:0 to 15:1) to yield racemic S5 (460 $\mathrm{mg}, 0.86 \mathrm{mmol}, 86 \%)$ as a clear and colorless oil.

S5: $\mathrm{R}_{f}$ (Hexanes:EtOAc, 15:1): 0.61; ${ }^{1} \mathrm{H}$ NMR $\left(400 \mathrm{MHz}, \mathrm{CDCl}_{3}\right) \delta 6.78-6.73(\mathrm{~m}, 1 \mathrm{H}), 6.73$ $6.67(\mathrm{~m}, 1 \mathrm{H}), 4.70(\mathrm{~d}, J=12.5 \mathrm{~Hz}, 1 \mathrm{H}), 4.54(\mathrm{~d}, J=12.5 \mathrm{~Hz}, 1 \mathrm{H}), 3.75(\mathrm{dd}, J=4.7,9.4 \mathrm{~Hz}, 2$ H), 3.64 (d, $J=9.4$ Hz, 2 H), 3.45 (br. s., 1 H), 3.37 (br. s., $1 \mathrm{H}), 2.28$ - 2.08 (m, $2 \mathrm{H}), 1.98$ - 1.85 (m, $2 \mathrm{H}), 1.45$ - $1.24(\mathrm{~m}, 4 \mathrm{H}), 1.24-1.14(\mathrm{~m}, 2 \mathrm{H}), 1.10(\mathrm{~s}, 3 \mathrm{H}), 0.91-0.85(\mathrm{~m}, 21 \mathrm{H}), 0.03(\mathrm{~s}$, $12 \mathrm{H}) \mathrm{ppm} ;{ }^{13} \mathrm{C}$ NMR $\left(100 \mathrm{MHz}, \mathrm{CDCl}_{3}\right) \delta 175.1,154.7,142.9,141.9,141.2,71.2,64.2,60.4$, 53.7, 52.0, 50.4, 31.4, 28.3, 26.9, 25.8, 22.5, 18.2, 17.1, 14.0, -5.6 ppm; IR (ATR) 2955, 2929, 2884, 2857, 1732, 1471, 1250, 1083, 833, $774 \mathrm{~cm}^{-1}$; HRMS (ES+) Exact mass cald. for $\mathrm{C}_{30} \mathrm{H}_{56} \mathrm{NaO}_{4} \mathrm{Si}_{2}[\mathrm{M}+\mathrm{Na}]^{+}: 559.3609$, found: 559.3629 .

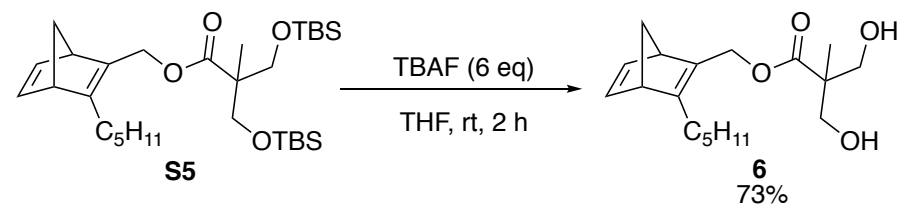

(3-pentylbicyclo[2.2.1]hepta-2,5-dien-2-yl)methyl 3-hydroxy-2-(hydroxymethyl)-2methylpropanoate (6):

A solution of tetra- $n$-butylammonium fluoride (TBAF) in THF (1 M, $4.9 \mathrm{~mL}, 4.90 \mathrm{mmol}, 6$ eq.) was added to a solution of $\mathbf{S 5}$ (440 $\mathrm{mg}, 0.819 \mathrm{mmol}, 1 \mathrm{eq}$ ) in THF $(12 \mathrm{~mL})$. After $2 \mathrm{~h}$, the reaction was deemed complete by NMR, and the solvent was removed. The residue was suspended in $\mathrm{Et}_{2} \mathrm{O}$ $(100 \mathrm{~mL})$, washed with a saturated solution of $\mathrm{NH}_{4} \mathrm{Cl}(50 \mathrm{~mL})$, then brine $(50 \mathrm{~mL})$. The organic layer was dried over $\mathrm{MgSO}_{4}$ and the solvent removed. The residue was subjected to flash column chromatography (hexanes:EtOAc, 1:1) to yield racemic $6(185 \mathrm{mg}, 0.600 \mathrm{mmol}, 73 \%)$ as a clear and colorless oil.

6: $\mathrm{R}_{f}$ (Hexanes:EtOAc, $\left.1: 1\right): 0.31 ;{ }^{1} \mathrm{H}$ NMR $\left(400 \mathrm{MHz}, \mathrm{CDCl}_{3}\right) \delta 6.80-6.74(\mathrm{~m}, 1 \mathrm{H}), 6.74$ $6.68(\mathrm{~m}, 1 \mathrm{H}), 4.84(\mathrm{~d}, J=12.1 \mathrm{~Hz}, 1 \mathrm{H}), 4.70(\mathrm{~d}, J=12.1 \mathrm{~Hz}, 1 \mathrm{H}), 3.88(\mathrm{dd}, J=6.1,11.2 \mathrm{~Hz}, 2$ H), 3.77 - 3.67 (m, 2 H), 3.46 (br. s., $1 \mathrm{H}$ ), 3.39 (br. s., $1 \mathrm{H}$ ), 2.79 (t, $J=6.5 \mathrm{~Hz}, 2 \mathrm{H}), 2.30$ - 2.12 (m, $2 \mathrm{H}), 1.98-1.89$ (m, $2 \mathrm{H}), 1.48-1.24(\mathrm{~m}, 4 \mathrm{H}), 1.24-1.12(\mathrm{~m}, 2 \mathrm{H}), 1.06(\mathrm{~s}, 3 \mathrm{H}), 0.87(\mathrm{t}, J$ $=7.2 \mathrm{~Hz}, 3 \mathrm{H}) \mathrm{ppm} ;{ }^{13} \mathrm{C} \mathrm{NMR}\left(100 \mathrm{MHz}, \mathrm{CDCl}_{3}\right) \delta 175.9,156.3,142.8,142.0,140.6,71.6$, 
68.39, 68.37, 61.1, 53.8, 52.2, 49.3, 31.4, 28.4, 26.8, 22.5, 17.2, 14.0 ppm; IR (ATR) 3420, 2959, 2930, 2864, 1717, 1460, 1378, 1293, 1219, 1118, 1035, 909, $730 \mathrm{~cm}^{-1}$; HRMS (ES+) Exact mass cald. for $\mathrm{C}_{18} \mathrm{H}_{28} \mathrm{NaO}_{4}^{+}[\mathrm{M}+\mathrm{Na}]^{+}: 331.1880$, found: 331.1884 .

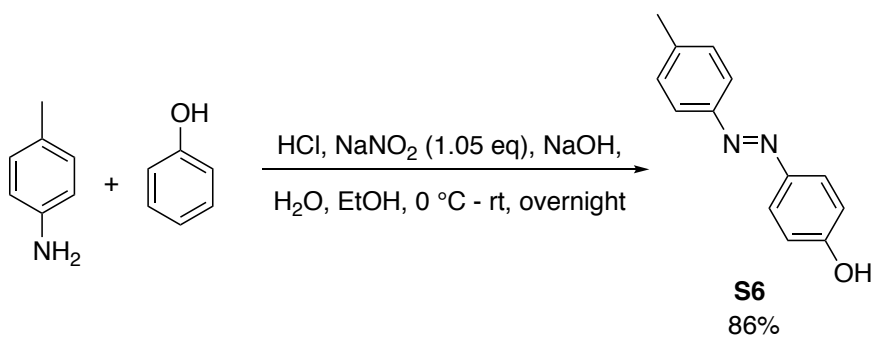

(E)-4-(p-tolyldiazenyl)phenol (S6):

$p$-Toluidine ( $2.0 \mathrm{~g}, 18.0 \mathrm{mmol}, 1.00$ eq.) was added to $50 \mathrm{~mL}$ water and cooled to $0{ }^{\circ} \mathrm{C}$. Concentrated $\mathrm{HCl}(13 \mathrm{M}, 5 \mathrm{~mL})$ was added dropwise while stirring. Once $p$-toluidine was fully dissolved, a solution of sodium nitrite (1.3 g, $18.9 \mathrm{mmol}, 1.05$ eq.) in water (40 mL) was added dropwise. The resulting solution was stirred at $0{ }^{\circ} \mathrm{C}$ for $30 \mathrm{~min}$. In a separate flask, phenol $(1.7 \mathrm{~g}$, $18.0 \mathrm{mmol}, 1.00$ eq.) was dissolved in sodium hydroxide solution $(7.6 \mathrm{~g}, 18.9 \mathrm{mmol}, 1.05 \mathrm{eq}$. in a mixture of $80 \mathrm{~mL}$ water and $20 \mathrm{~mL}$ ethanol) and cooled to $0^{\circ} \mathrm{C}$. The diazonium solution was then added dropwise over $30 \mathrm{~min}$ to the phenoxide solution at $0{ }^{\circ} \mathrm{C}$. The resulting mixture was stirred overnight. The product was collected by vacuum filtration to afford $\mathbf{S 6}$ as a bright yellow solid $(3.3 \mathrm{~g}, 15.5 \mathrm{mmol}, 86 \%)$. Spectral data matched that of reported literature. ${ }^{4}$

S6: ${ }^{1} \mathrm{H}$ NMR $\left(400 \mathrm{MHz}, \mathrm{CDCl}_{3}\right) \delta 7.86(\mathrm{~d}, J=8.9 \mathrm{~Hz}, 2 \mathrm{H}), 7.78(\mathrm{~d}, J=8.3 \mathrm{~Hz}, 2 \mathrm{H}), 7.30(\mathrm{~d}, J=$ $8.1 \mathrm{~Hz}, 2 \mathrm{H}), 6.94$ (d, J=8.9 Hz, $2 \mathrm{H}$ ), 5.09 (br. s., $1 \mathrm{H}$ ), 2.43 (s, $3 \mathrm{H})$ ppm.

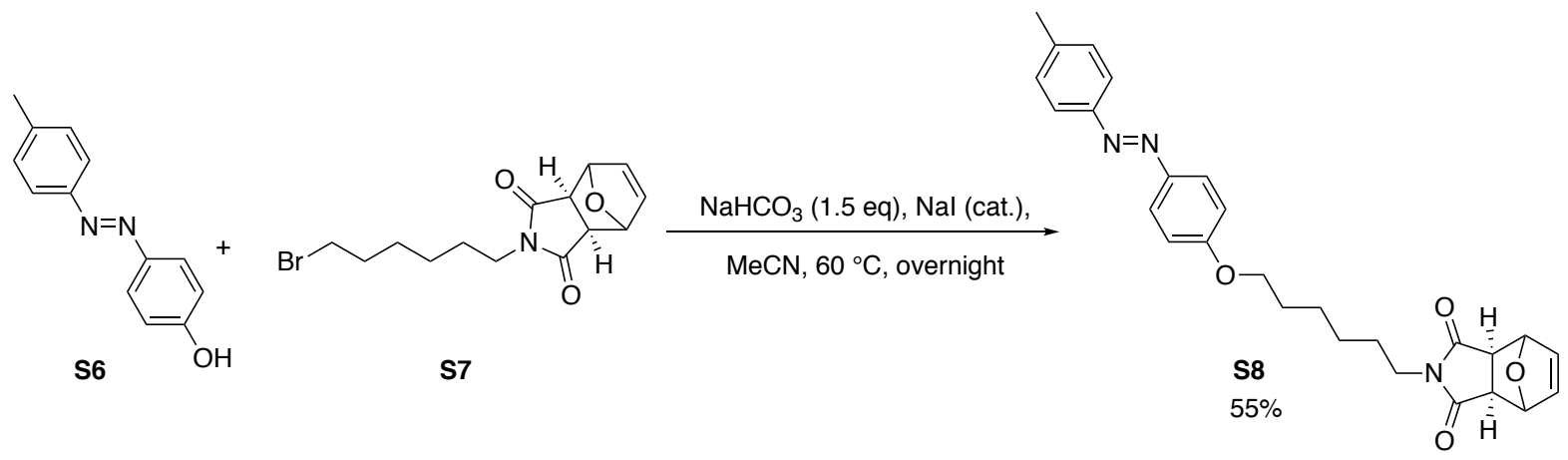

(3aR,7aS)-2-(6-(4-((E)-p-tolyldiazenyl)phenoxy)hexyl)-3a,4,7,7a-tetrahydro-1H-4,7epoxyisoindole-1,3(2H)-dione (S8):

S6 (1.00 g, $4.71 \mathrm{mmol}, 1.00$ eq.), $\mathbf{S} 7^{5}$ (1.90 g, $5.79 \mathrm{mmol}, 1.25$ eq.) and sodium bicarbonate ( $0.95 \mathrm{~g}$, $6.9 \mathrm{mmol}, 1.5$ eq.) were added to $10 \mathrm{~mL}$ acetonitrile $(\mathrm{MeCN})$. The heterogenous mixture was stirred with catalytic sodium iodide $(10 \mathrm{mg})$ overnight at $60^{\circ} \mathrm{C}$. The mixture was brought to $\mathrm{rt}$, and the solids were removed by filtration. The product was precipitated by adding $20 \mathrm{~mL}$ of water to the filtrate and collected by vacuum filtration to afford $\mathbf{S 8}(1.18 \mathrm{~g}, 2.57 \mathrm{mmol}, 55 \%)$ as a yelloworange powder.

S8: ${ }^{1} \mathrm{H}$ NMR $\left(400 \mathrm{MHz}, \mathrm{CDCl}_{3}\right) \delta 7.88(\mathrm{~d}, J=8.9 \mathrm{~Hz}, 2 \mathrm{H}), 7.78(\mathrm{~d}, J=8.2 \mathrm{~Hz}, 2 \mathrm{H}), 7.29(\mathrm{~d}, J=$ $8.2 \mathrm{~Hz}, 2 \mathrm{H}), 6.98(\mathrm{~d}, J=8.9 \mathrm{~Hz}, 2 \mathrm{H}), 6.50(\mathrm{~s}, 2 \mathrm{H}), 5.25(\mathrm{~s}, 2 \mathrm{H}), 4.01$ (t, $J=6.5 \mathrm{~Hz}, 2 \mathrm{H}), 3.49$ S8 
(t, $J=7.3 \mathrm{~Hz}, 2 \mathrm{H}), 2.82(\mathrm{~s}, 2 \mathrm{H}), 2.42(\mathrm{~s}, 3 \mathrm{H}), 1.83-1.77(\mathrm{~m}, 2 \mathrm{H}), 1.64-1.58(\mathrm{~m}, 2 \mathrm{H}), 1.53-$ $1.47(\mathrm{~m}, 2 \mathrm{H}), 1.39-1.33(\mathrm{~m}, 2 \mathrm{H}) \mathrm{ppm} ;{ }^{13} \mathrm{C}$ NMR $\left(100 \mathrm{MHz}, \mathrm{CDCl}_{3}\right) \delta 176.3,161.4,150.8$, $146.9,140.7,136.5,129.7,124.5,122.5,114.6,80.9,68.0,47.4,38.8,29.0,27.5,26.3,25.5$, 21.4 ppm; IR (ATR) 3442, 3007, 2944, 2856, 1771, 1691, 1399, 1257, 1137, 845, 823, $548 \mathrm{~cm}^{-1}$; HRMS (ES+) Exact mass cald. for $\mathrm{C}_{27} \mathrm{H}_{29} \mathrm{~N}_{3} \mathrm{O}_{4} \mathrm{Na}^{+}[\mathrm{M}+\mathrm{Na}]^{+}$: 482.2056, found: 482.2058.

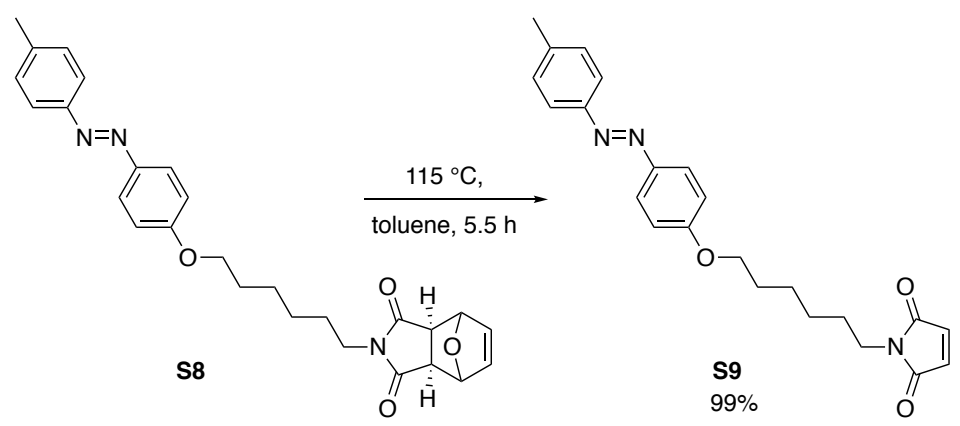

(E)-1-(6-(4-(p-tolyldiazenyl)phenoxy)hexyl)-1H-pyrrole-2,5-dione (S9):

S8 $(0.150 \mathrm{~g}, 0.3 \mathrm{mmol})$ was dissolved in $25 \mathrm{~mL}$ toluene and refluxed at $115{ }^{\circ} \mathrm{C}$ for $5.5 \mathrm{~h}$. The toluene was removed under reduced pressure to afford $\mathbf{S 9}(0.127 \mathrm{~g}, 0.3 \mathrm{mmol}, 99 \%)$ as a yelloworange powder.

S9: ${ }^{1} \mathrm{H}$ NMR $\left(400 \mathrm{MHz}, \mathrm{CDCl}_{3}\right) \delta 7.88(\mathrm{~d}, J=9.0 \mathrm{~Hz}, 2 \mathrm{H}), 7.78(\mathrm{~d}, J=8.3 \mathrm{~Hz}, 2 \mathrm{H}), 7.29(\mathrm{~d}, J=$ $8.1 \mathrm{~Hz}, 2 \mathrm{H}), 6.98$ (d, $J=9.0 \mathrm{~Hz}, 2 \mathrm{H}), 6.68(\mathrm{~s}, 2 \mathrm{H}), 4.02(\mathrm{t}, J=6.4 \mathrm{~Hz}, 2 \mathrm{H}), 3.54$ (t, $J=7.3 \mathrm{~Hz}$, $2 \mathrm{H}), 2.43(\mathrm{~s}, 3 \mathrm{H}), 1.84-1.77(\mathrm{~m}, 2 \mathrm{H}), 1.68-1.58(\mathrm{~m}, 2 \mathrm{H}), 1.56-1.48(\mathrm{~m}, 2 \mathrm{H}), 1.41-1.34(\mathrm{~m}$, $2 \mathrm{H}) \mathrm{ppm} ;{ }^{13} \mathrm{C} \mathrm{NMR}\left(100 \mathrm{MHz}, \mathrm{CDCl}_{3}\right) \delta 170.9,161.3,150.8,146.9,140.7,134.0,129.7,124.5$, 122.5, 114.6, 68.0, 37.8, 29.0, 28.5, 26.5, 25.6, 21.4 ppm; IR (ATR) 3373, 3097, 3025, 2945, 2913, 2857, 1697, 1407, 1247, 825, 692, $544 \mathrm{~cm}^{-1}$; HRMS (ES+) Exact mass cald. for $\mathrm{C}_{23} \mathrm{H}_{25} \mathrm{~N}_{3} \mathrm{O}_{3} \mathrm{Na}^{+}$ $[\mathrm{M}+\mathrm{Na}]^{+}:$414.1794, found: 414.1799 . 


\section{3. ${ }^{1} \mathrm{H}$ and ${ }^{13} \mathrm{C}$ NMR Spectra}

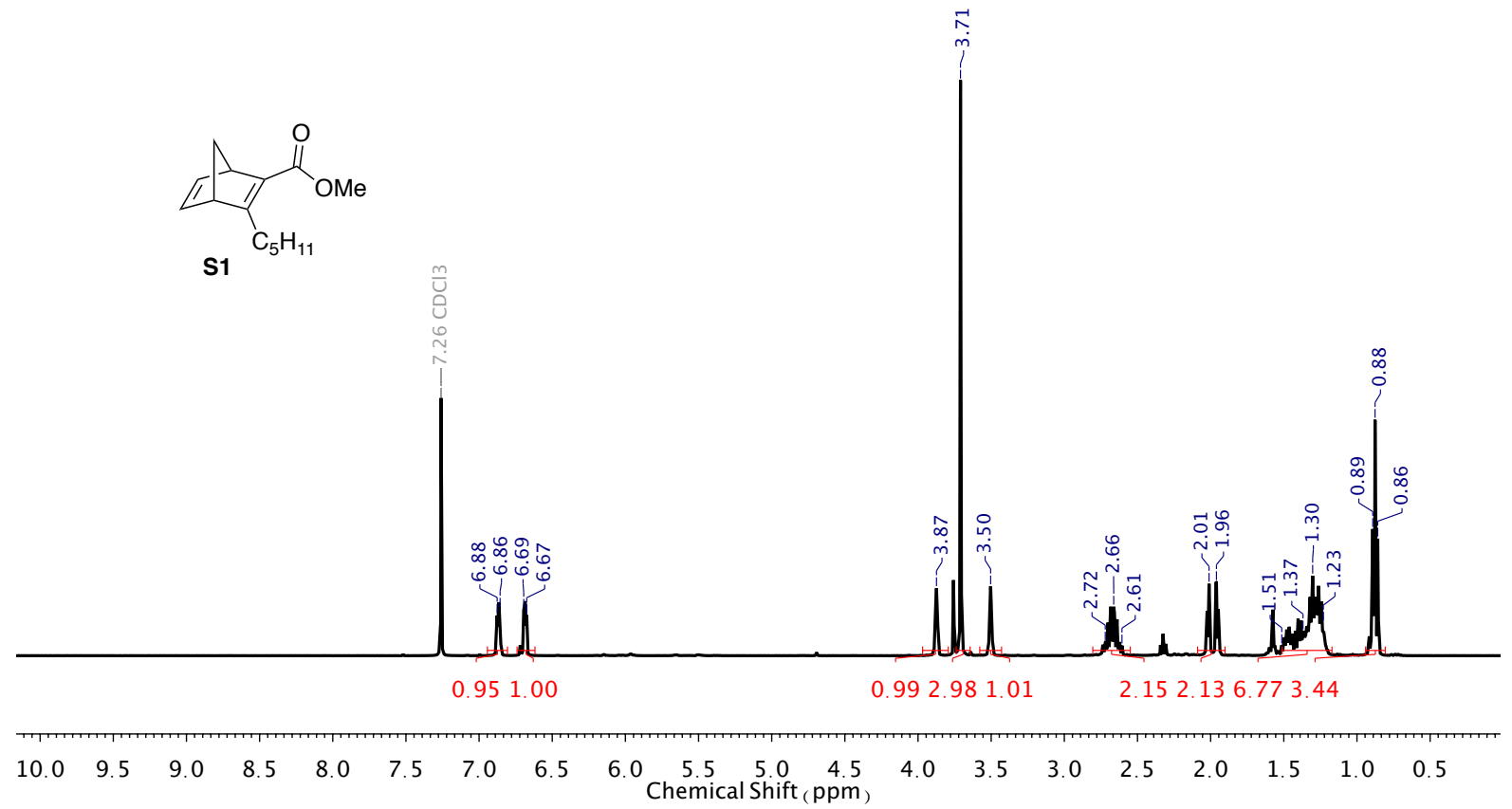

Figure S1. ${ }^{1} \mathrm{H} \mathrm{NMR}\left(400 \mathrm{MHz}, \mathrm{CDCl}_{3}\right) \mathbf{S 1}$

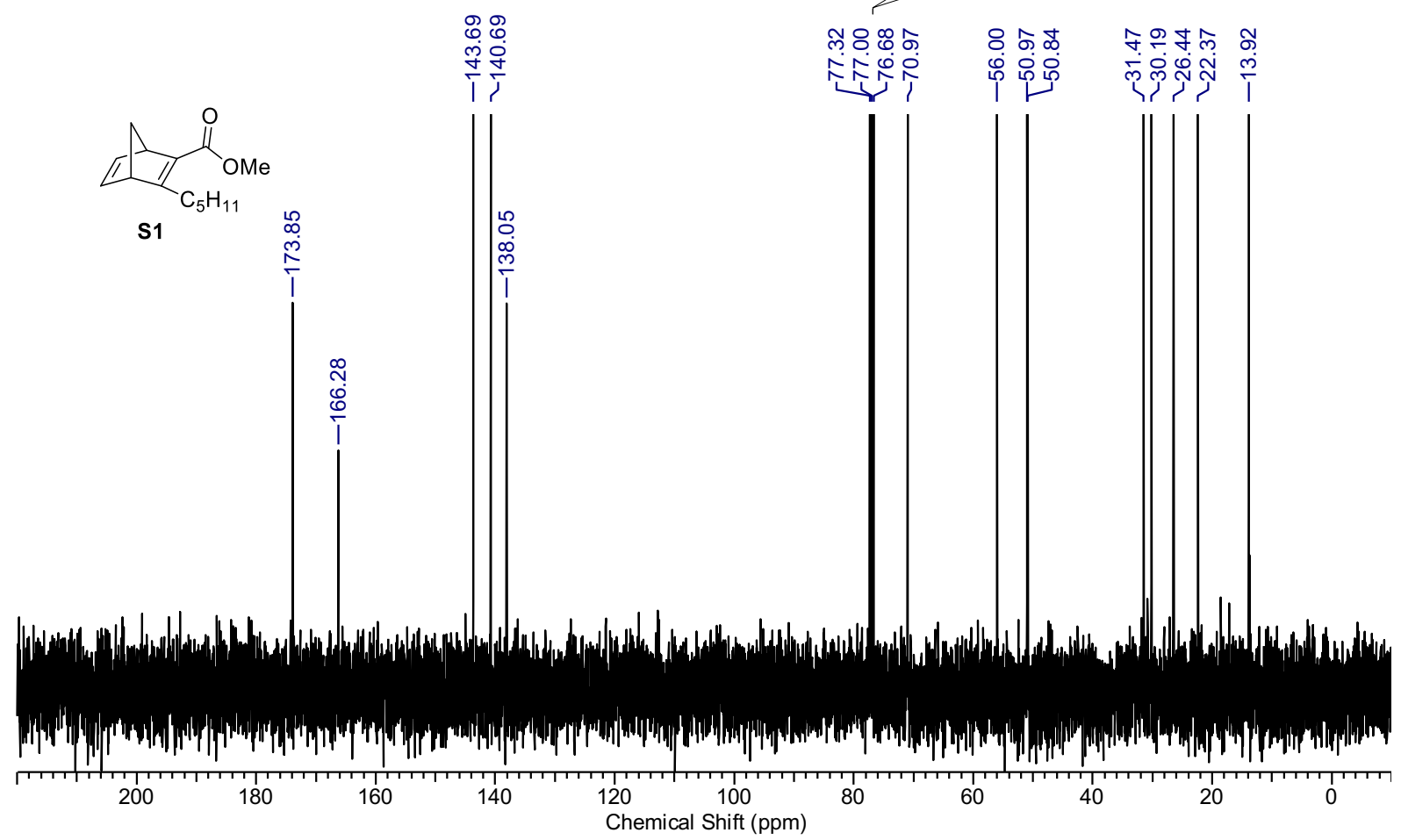

Figure S2. ${ }^{13} \mathrm{C}$ NMR $\left(100 \mathrm{MHz}, \mathrm{CDCl}_{3}\right) \mathbf{S 1}$ 


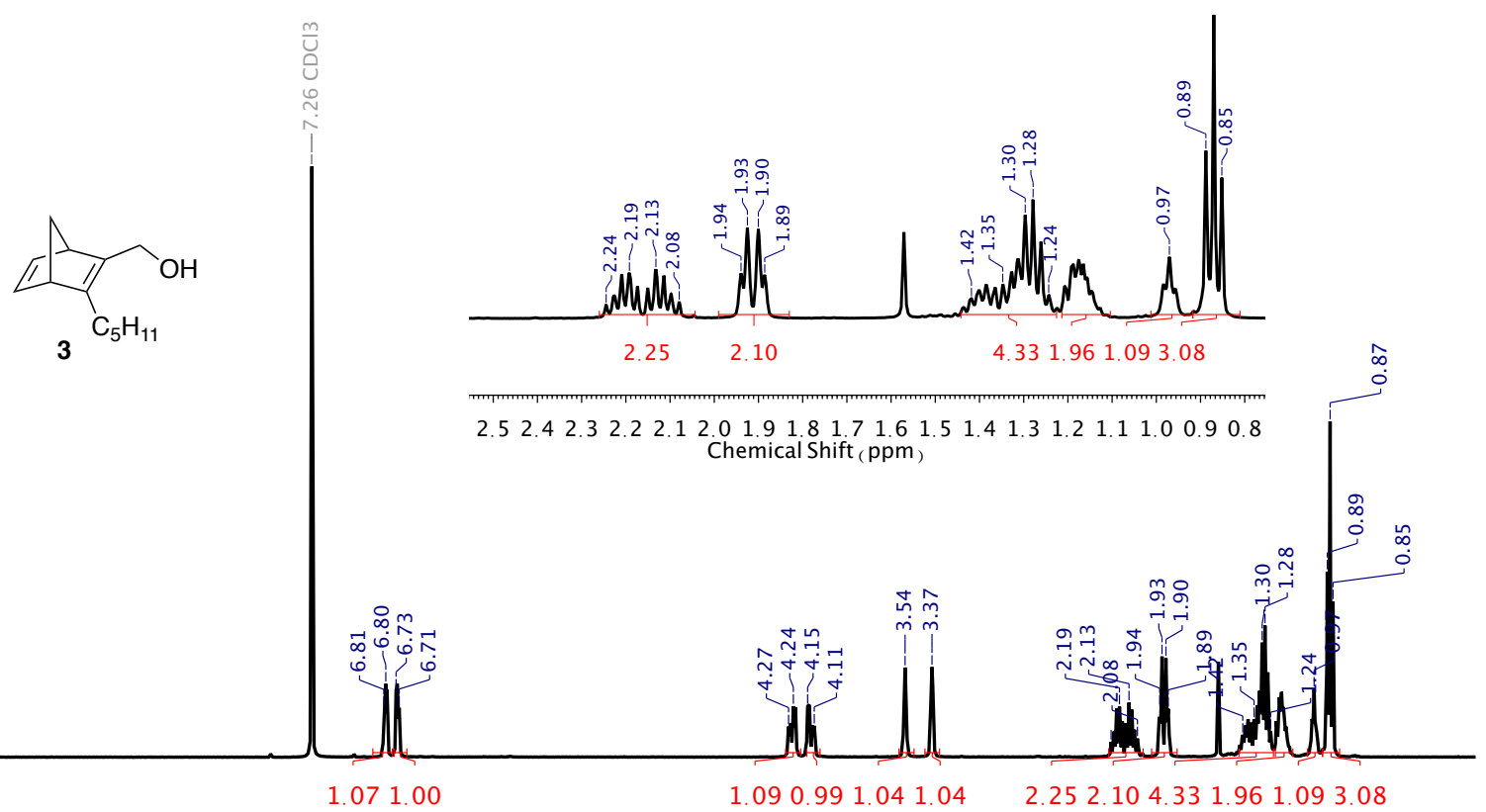

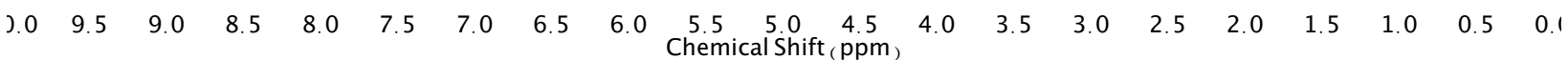

Figure S3. ${ }^{1} \mathrm{H}$ NMR $\left(400 \mathrm{MHz}, \mathrm{CDCl}_{3}\right) 3$

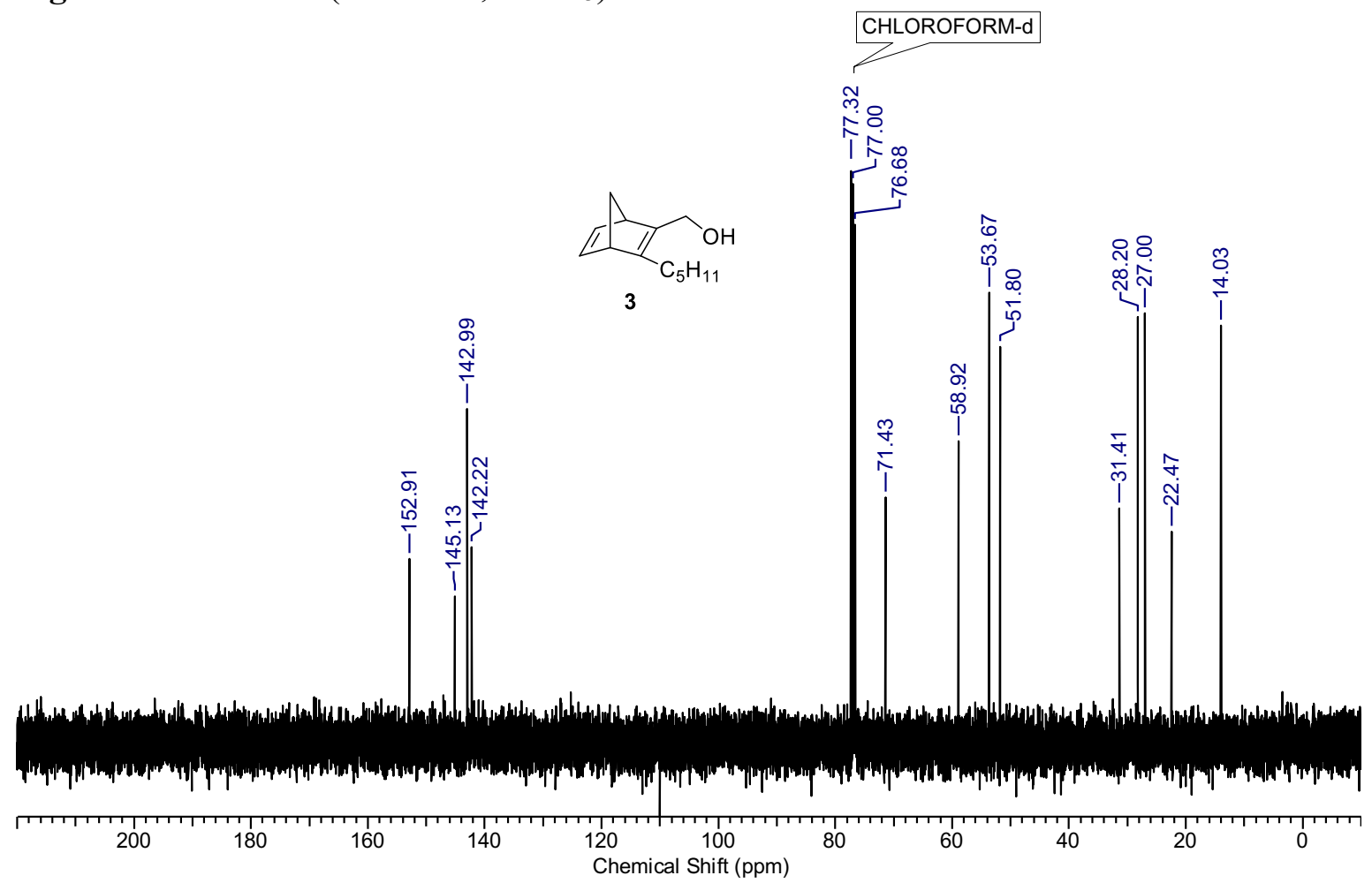

Figure S4. ${ }^{13} \mathrm{C}$ NMR $\left(100 \mathrm{MHz}, \mathrm{CDCl}_{3}\right) \mathbf{3}$ 


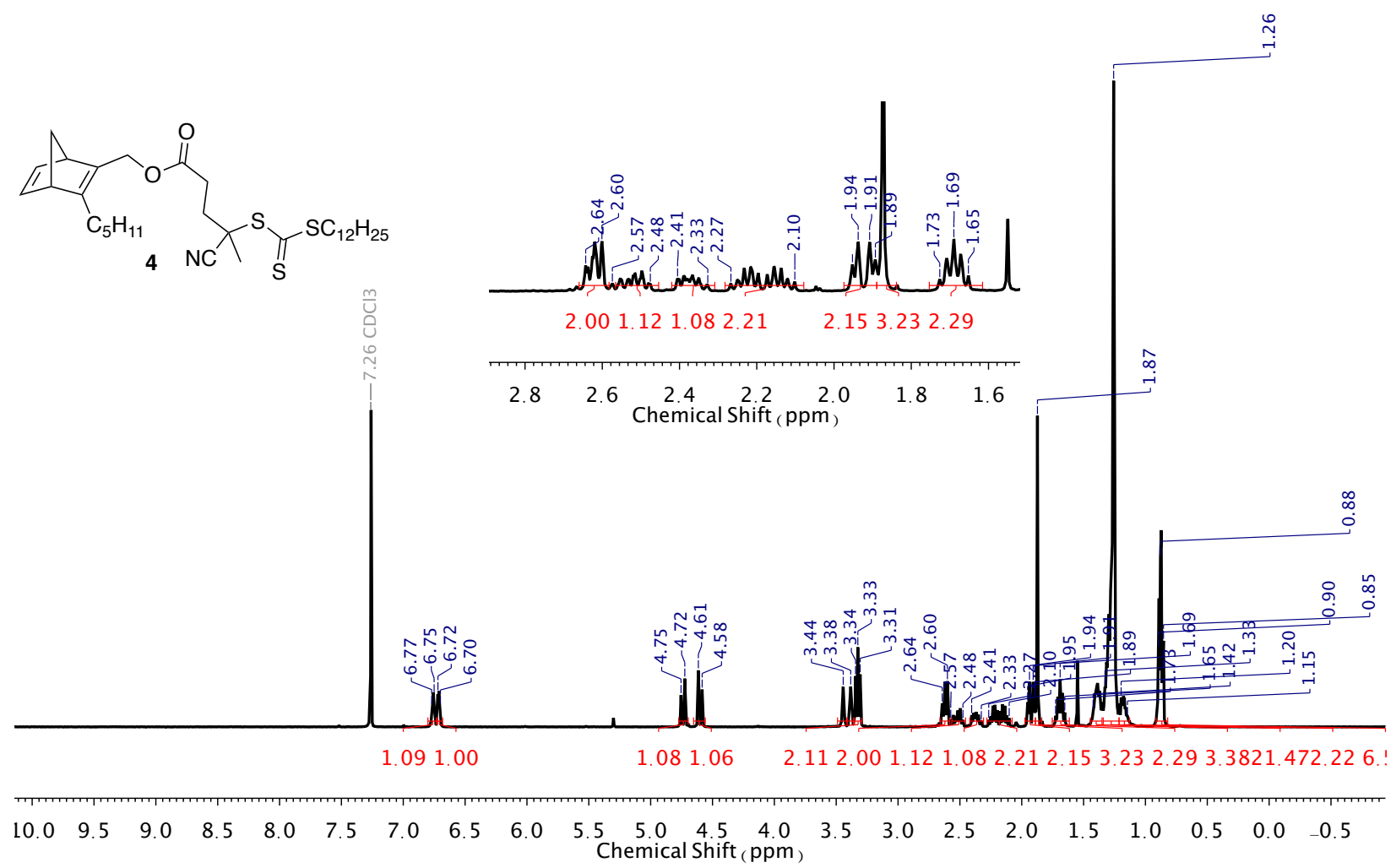

Figure S5. ${ }^{1} \mathrm{H}$ NMR (400 $\left.\mathrm{MHz}, \mathrm{CDCl}_{3}\right) 4$

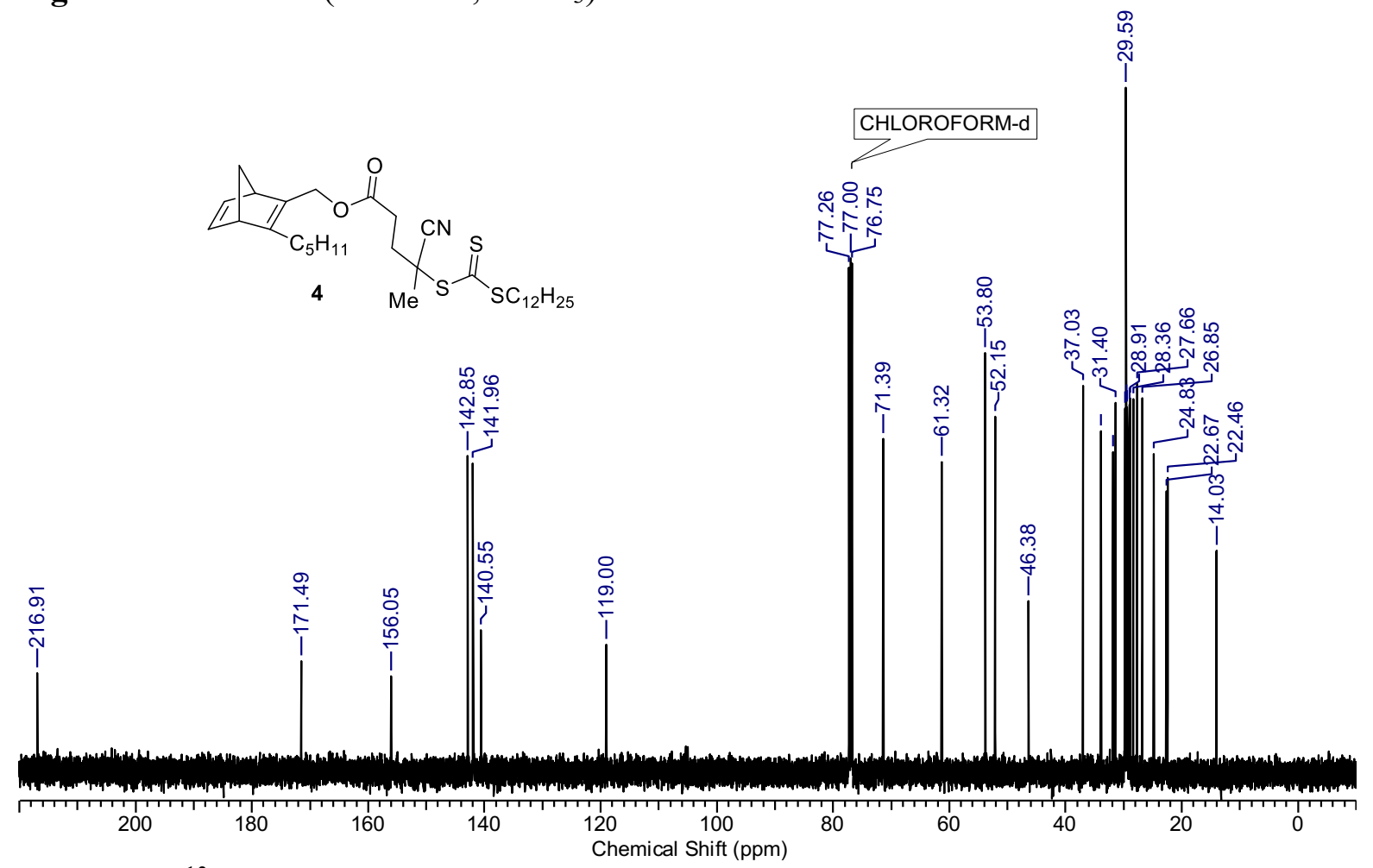

Figure S6. ${ }^{13} \mathrm{C} \mathrm{NMR}\left(125 \mathrm{MHz}, \mathrm{CDCl}_{3}\right) 4$ 


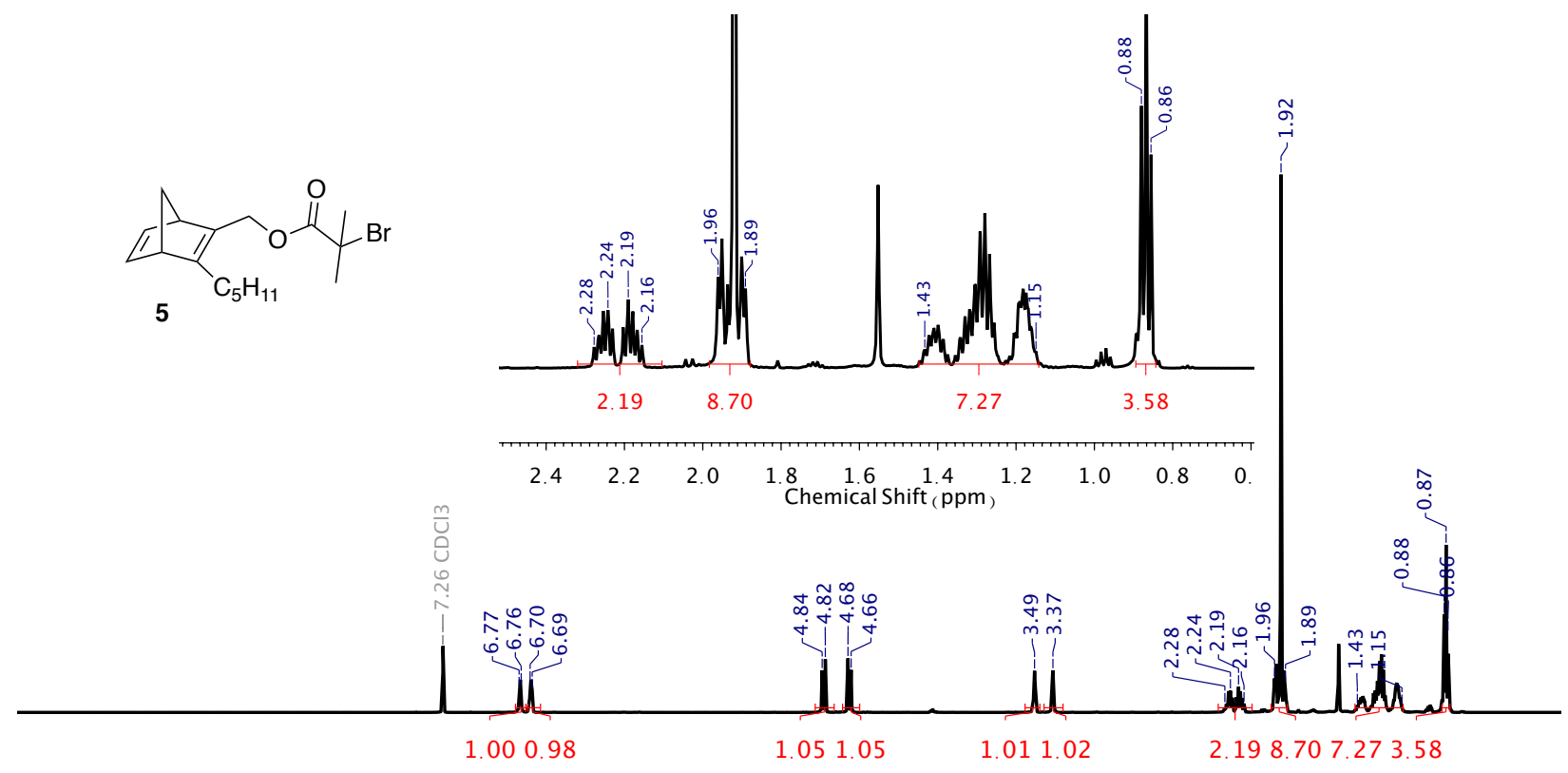

$$
\begin{array}{lllllllllllllllllll}
9.5 & 9.0 & 8.5 & 8.0 & 7.5 & 7.0 & 6.5 & 6.0 & \begin{array}{c}
5.5 \\
\text { Chemical Shift }(\mathrm{ppm})
\end{array} & \begin{array}{l}
4.0 \\
4.0
\end{array} & 3.5 & 3.0 & 2.5 & 2.0 & 1.5 & 1.0 & 0.5
\end{array}
$$

Figure S7. ${ }^{1} \mathrm{H} \mathrm{NMR}\left(400 \mathrm{MHz}, \mathrm{CDCl}_{3}\right) \mathbf{5}$<smiles>CC(Br)(Br)C(=O)OCC1=C([SeH])C2C=CC1C2</smiles>

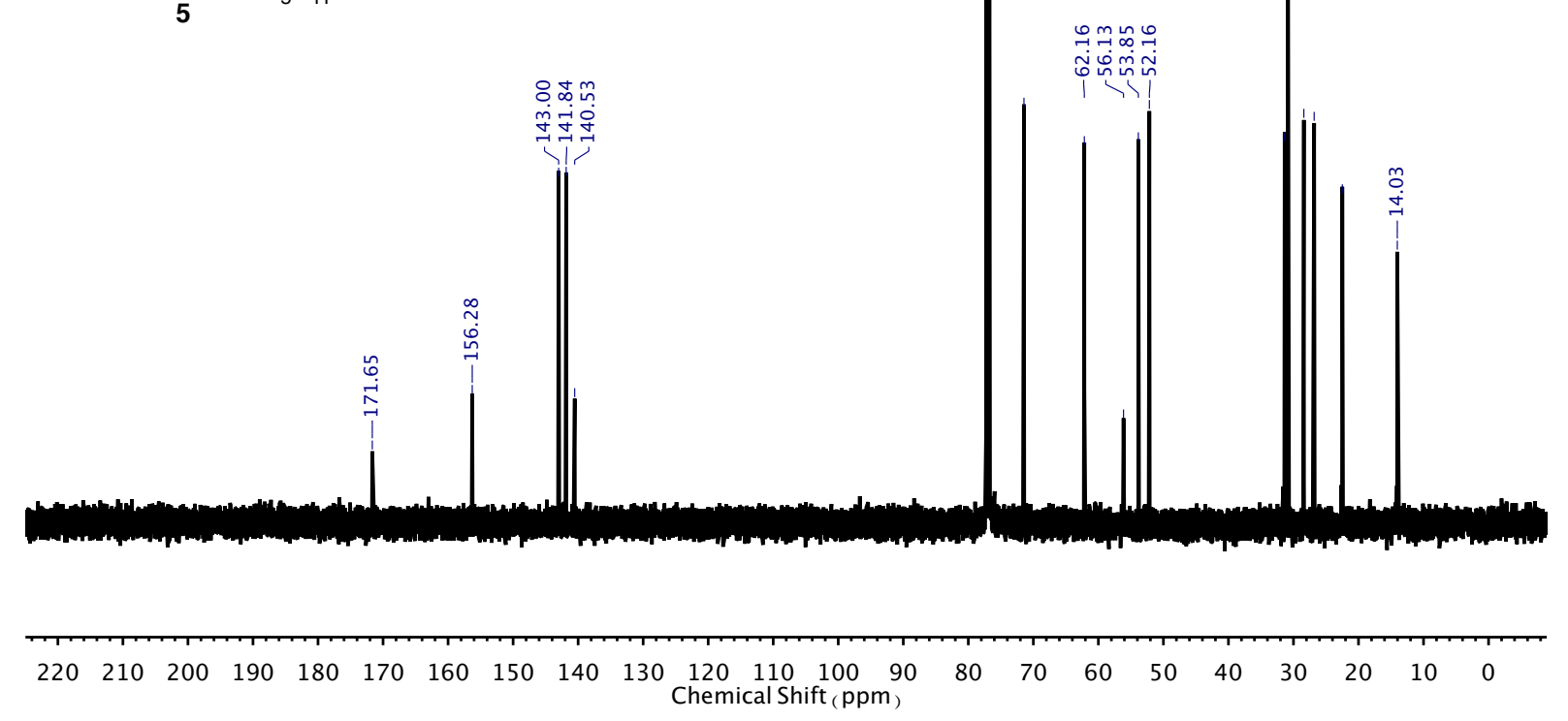

Figure S8. ${ }^{13} \mathrm{C} \mathrm{NMR}\left(100 \mathrm{MHz}, \mathrm{CDCl}_{3}\right) \mathbf{5}$

S13 


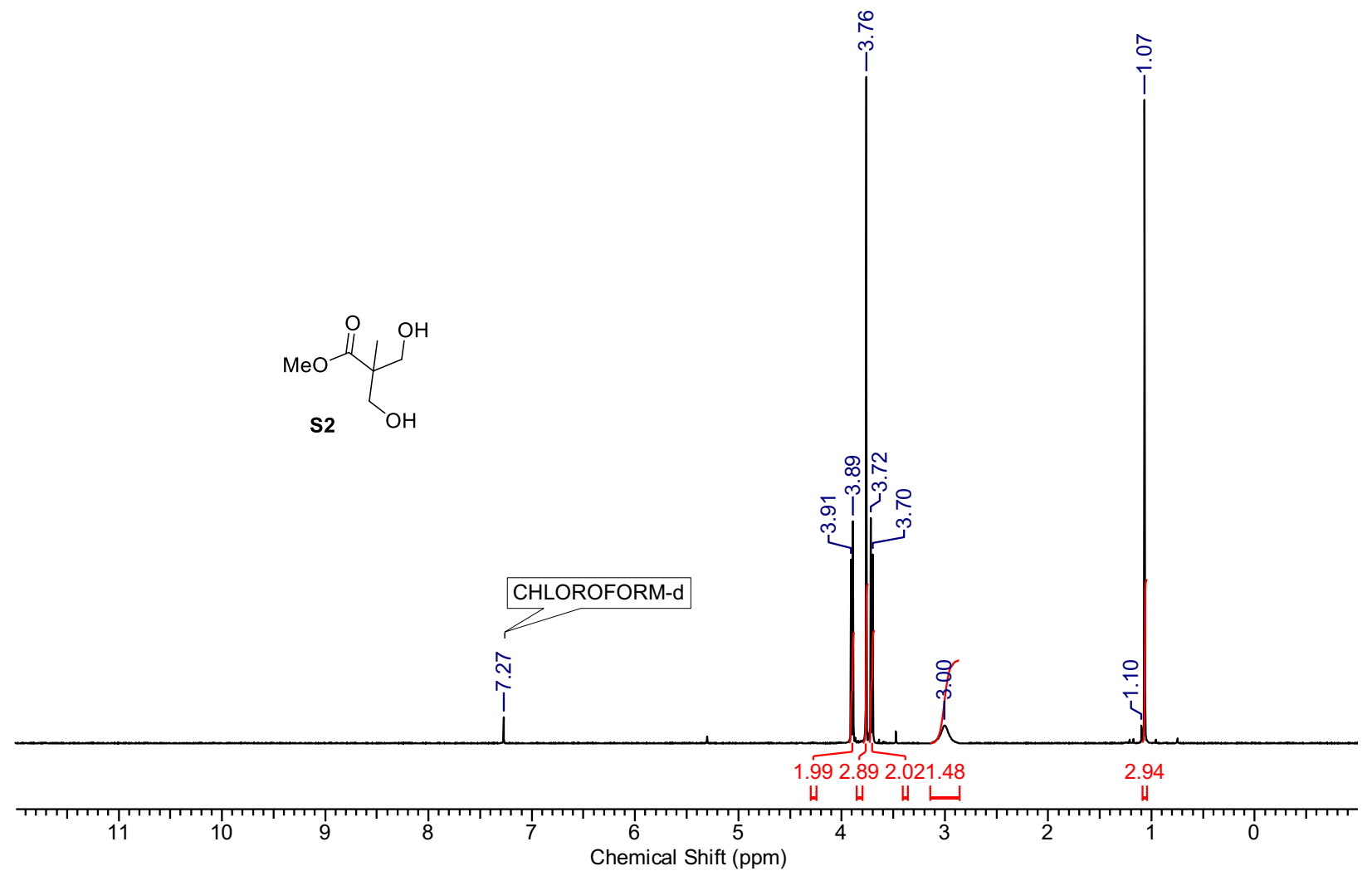

Figure S9. ${ }^{1} \mathrm{H} \mathrm{NMR}\left(600 \mathrm{MHz}, \mathrm{CDCl}_{3}\right)$ S2 


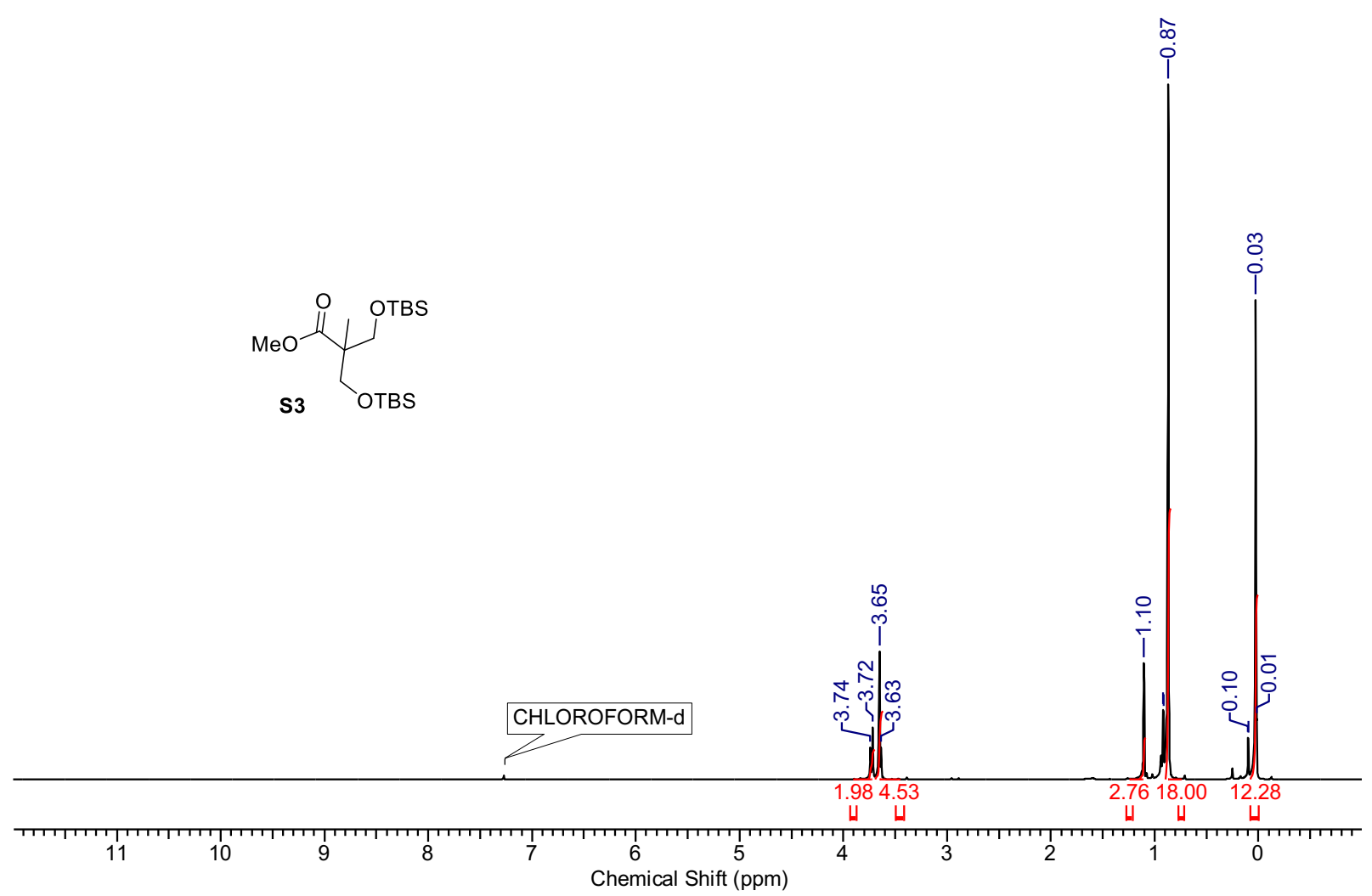

Figure S10. ${ }^{1} \mathrm{H} \mathrm{NMR}\left(400 \mathrm{MHz}, \mathrm{CDCl}_{3}\right) \mathbf{S 3}$
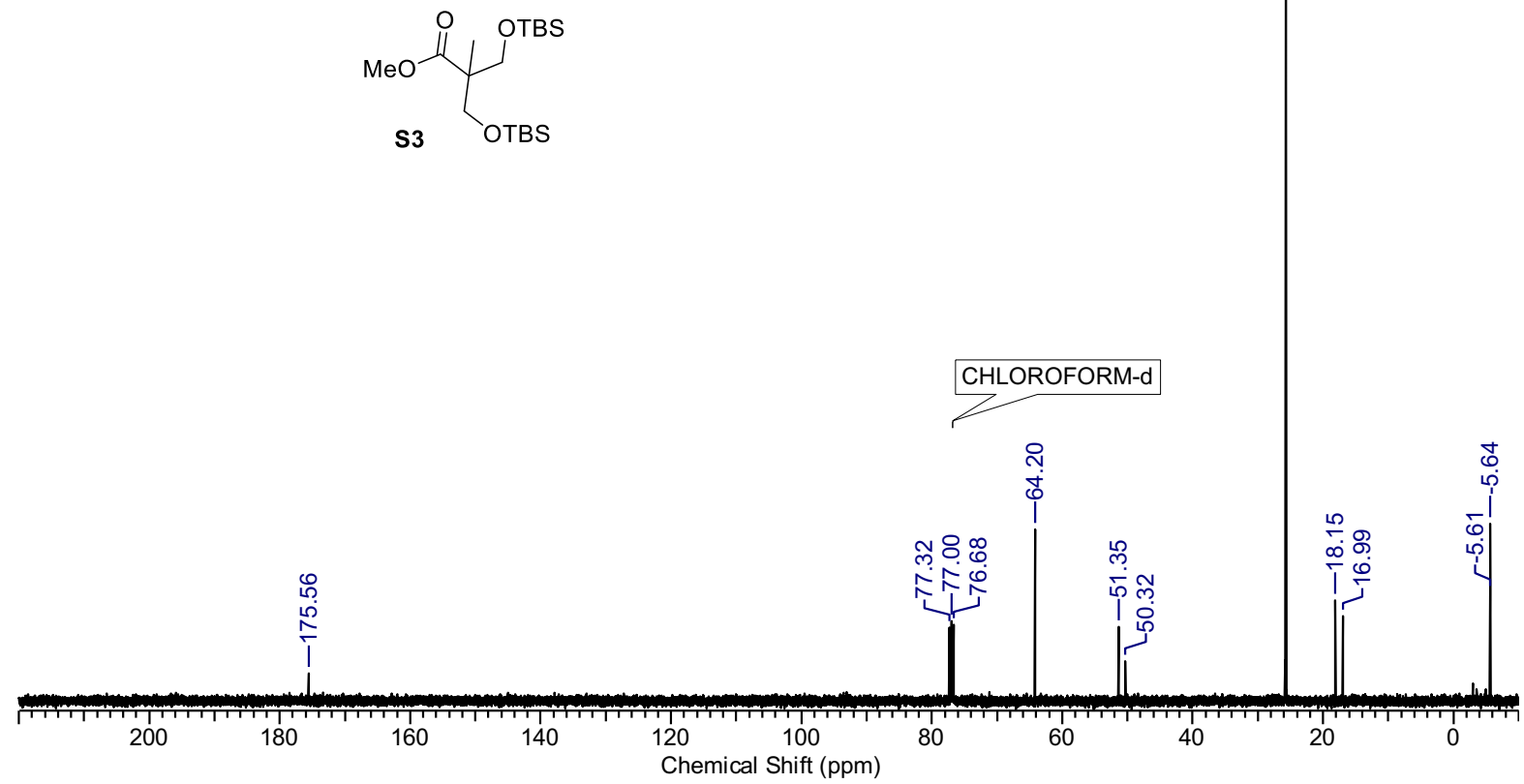

Figure S11. ${ }^{13} \mathrm{C}$ NMR (100 $\left.\mathrm{MHz}, \mathrm{CDCl}_{3}\right) \mathbf{S 3}$

S15 


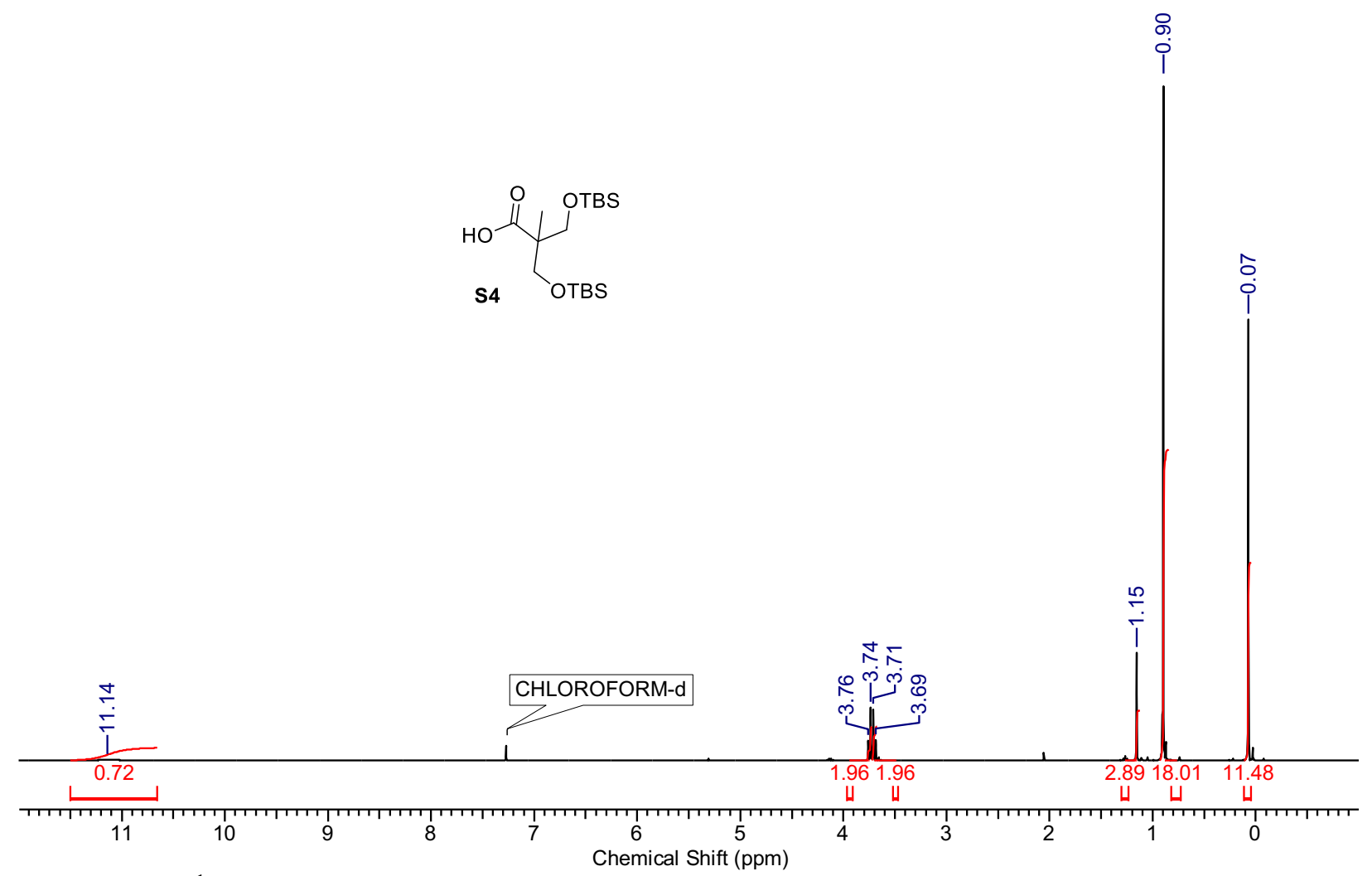

Figure $\mathbf{S 1 2} .{ }^{1} \mathrm{H}$ NMR (400 MHz, $\left.\mathrm{CDCl}_{3}\right) \mathbf{S 4}$

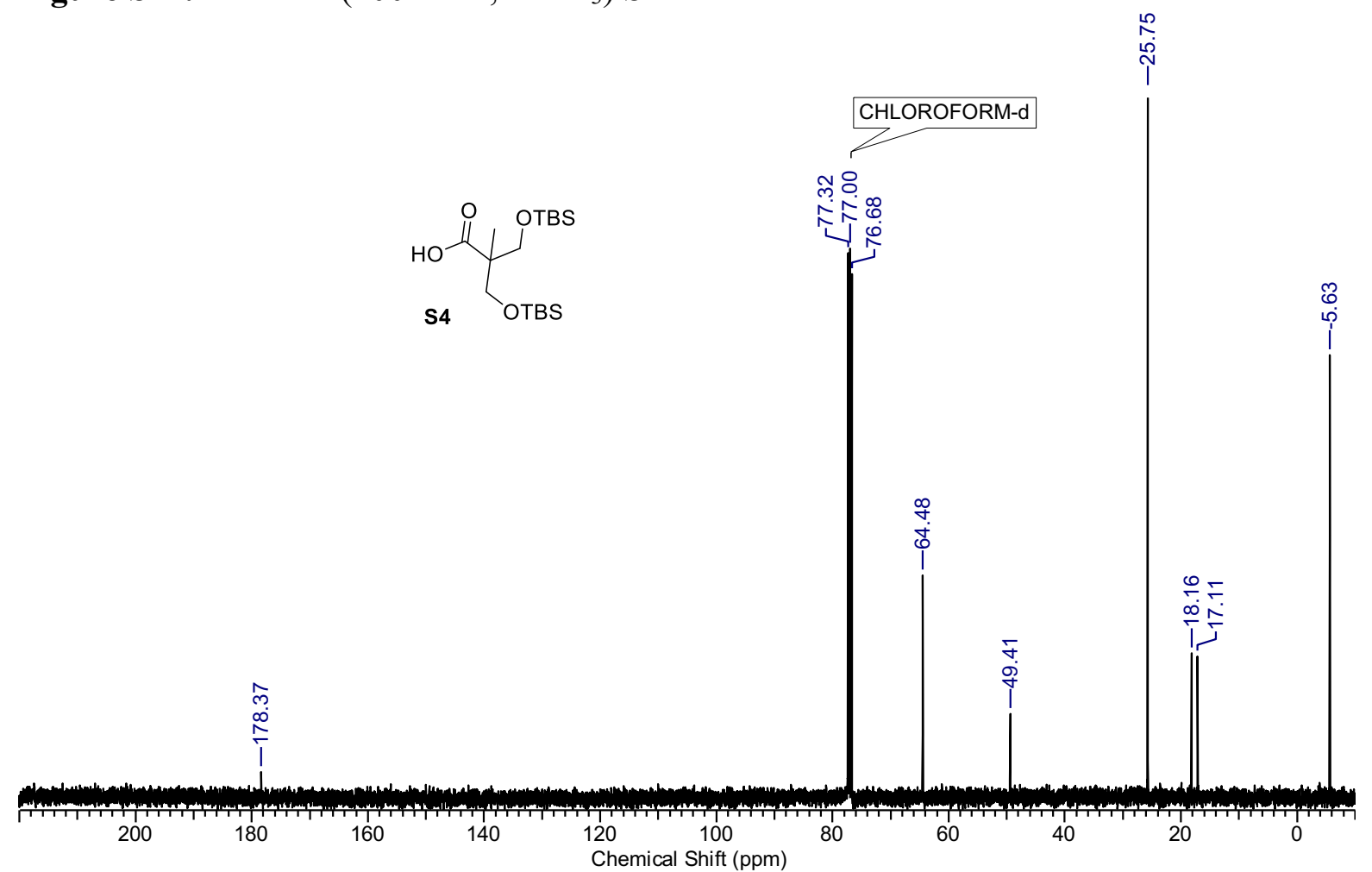

Figure S13. ${ }^{13} \mathrm{C}$ NMR (100 MHz, $\left.\mathrm{CDCl}_{3}\right) \mathbf{S 4}$

S16 

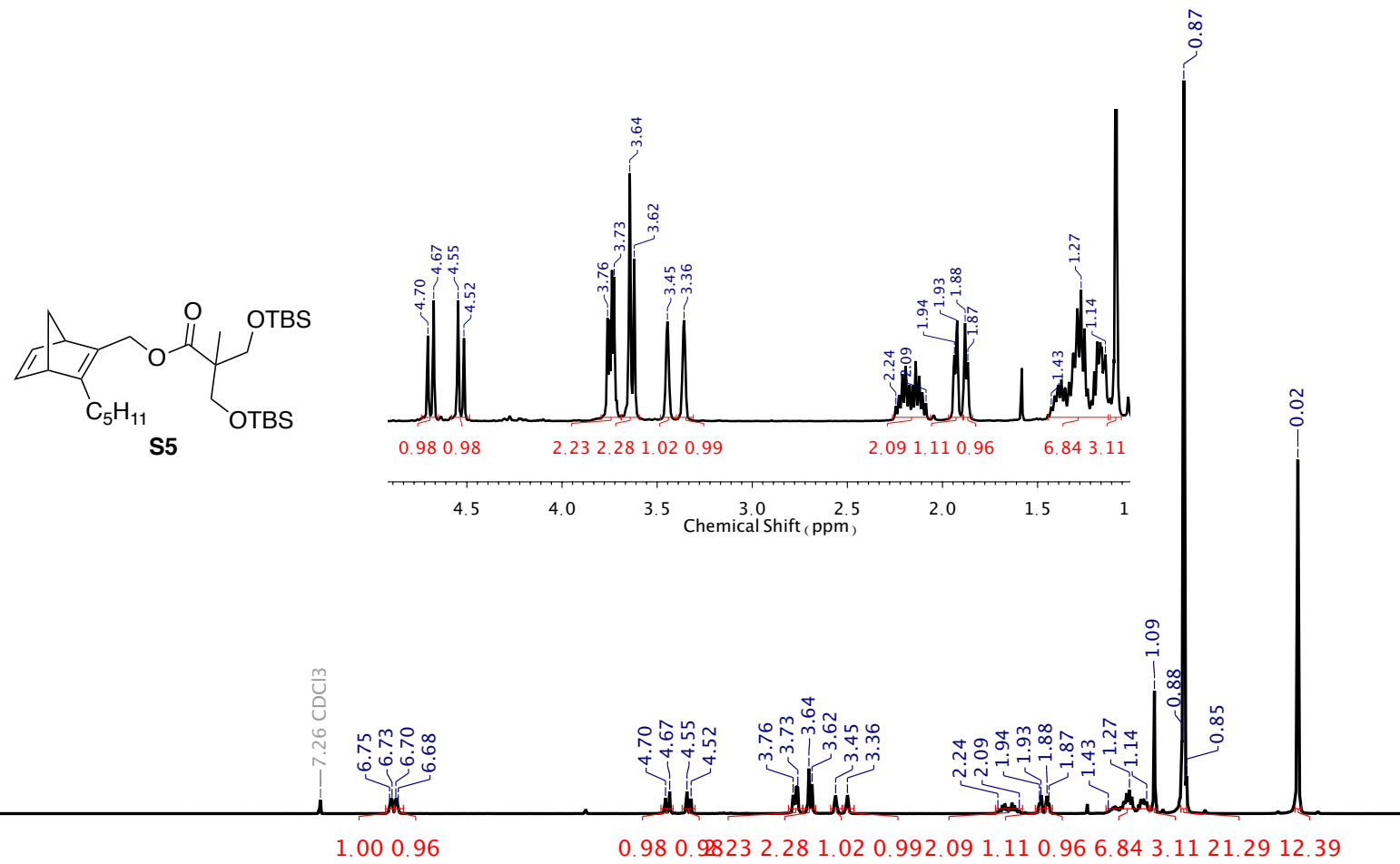

$\begin{array}{llllllllllllllllllllll}10.0 & 9.5 & 9.0 & 8.5 & 8.0 & 7.5 & 7.0 & 6.5 & 6.0 & \begin{array}{l}5.5 \\ \text { Chemical Shift }\end{array} \quad \begin{array}{l}4.0 \\ \text { ppm) }\end{array} & 3.5 & 3.0 & 2.5 & 2.0 & 1.5 & 1.0 & 0.5 & 0.0 & -0.5\end{array}$

Figure S14. ${ }^{1} \mathrm{H}$ NMR (400 $\left.\mathrm{MHz}, \mathrm{CDCl}_{3}\right) \mathbf{S 5}$

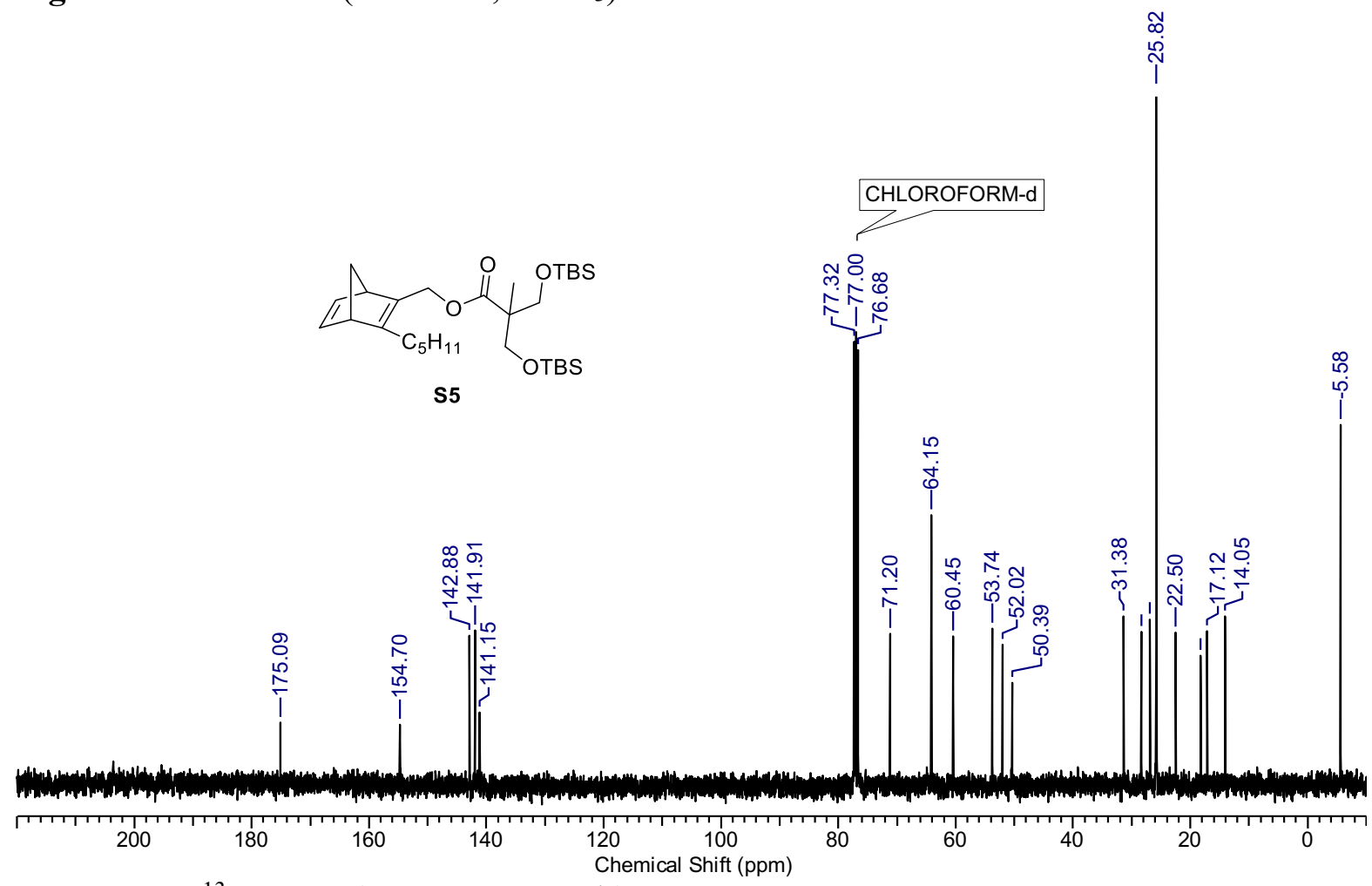

Figure S15. ${ }^{13} \mathrm{C}$ NMR (100 MHz, $\left.\mathrm{CDCl}_{3}\right) \mathbf{S 5}$

S17 

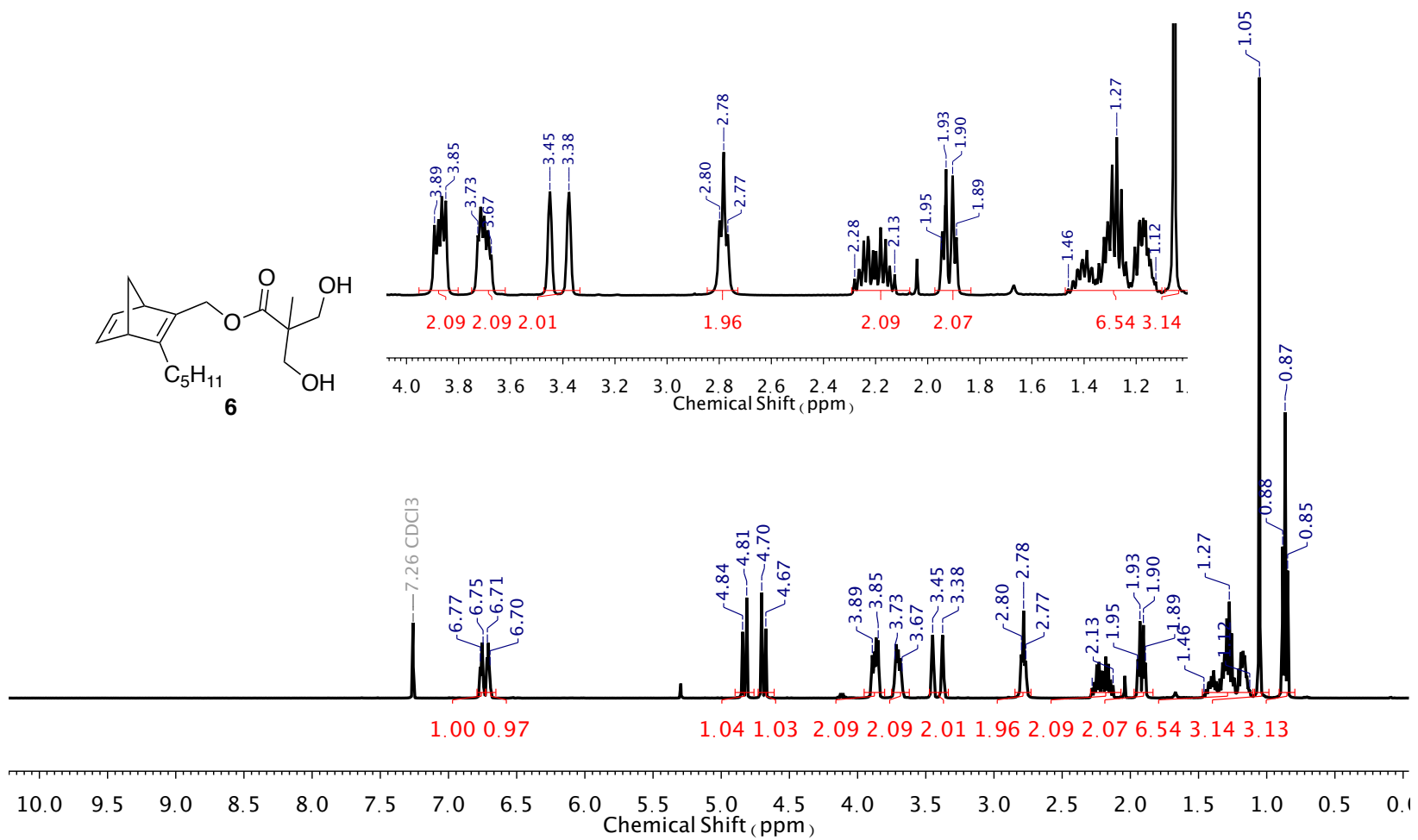

Figure S16. ${ }^{1} \mathrm{H}$ NMR $\left(400 \mathrm{MHz}, \mathrm{CDCl}_{3}\right) 6$

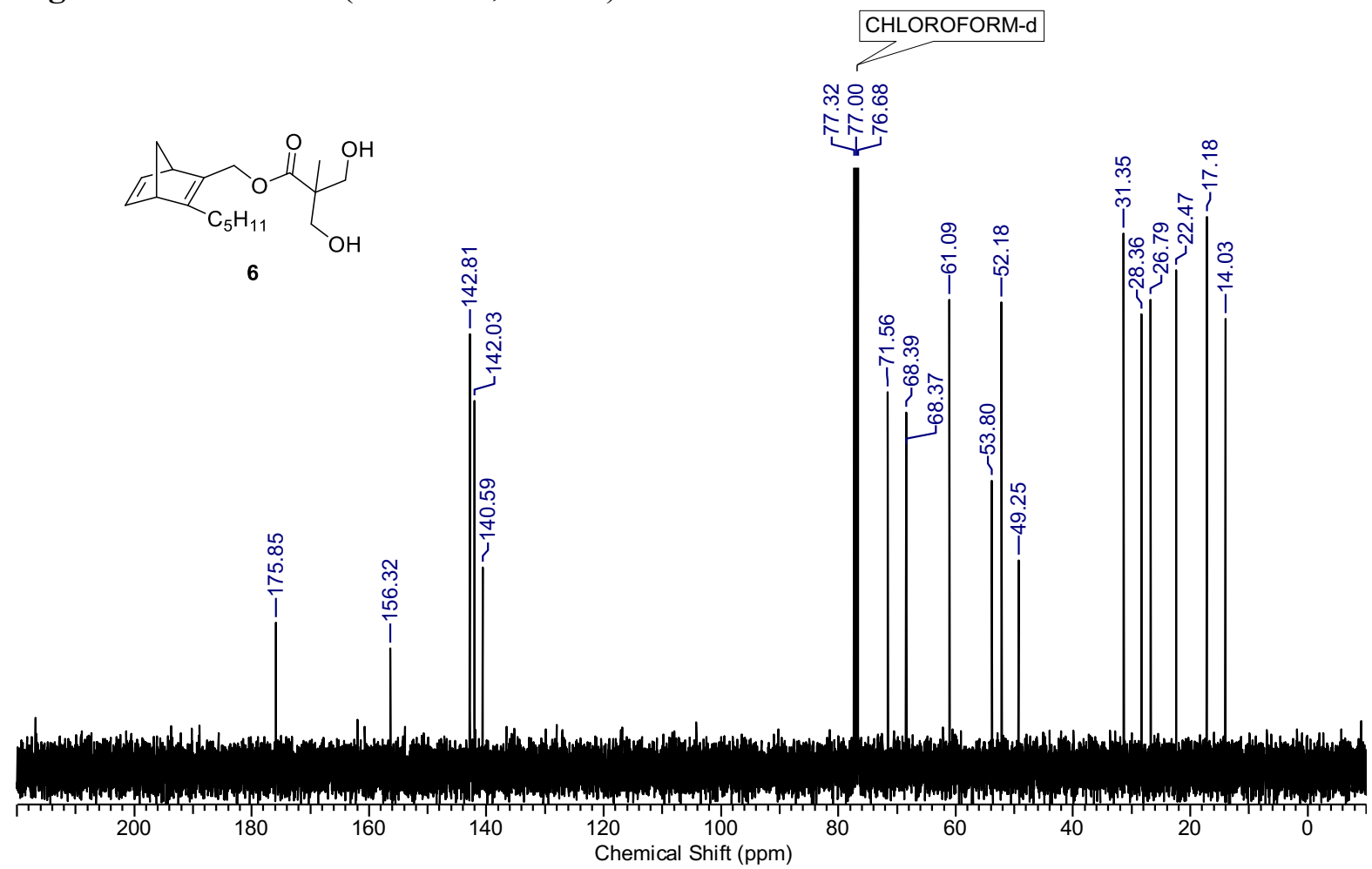

Figure S17. ${ }^{13} \mathrm{C}$ NMR $\left(100 \mathrm{MHz}, \mathrm{CDCl}_{3}\right) 6$ 


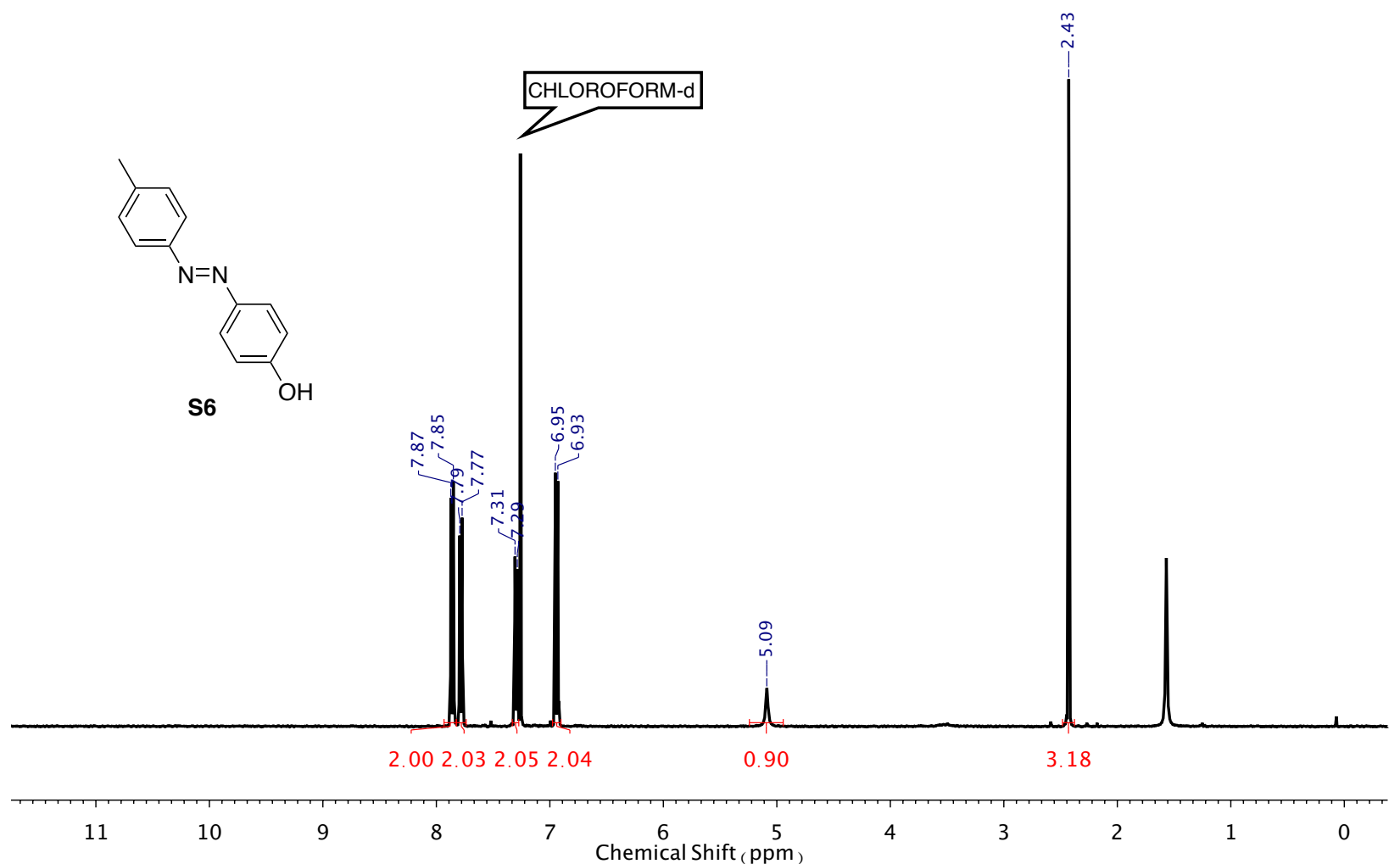

Figure S18. ${ }^{1} \mathrm{H} \mathrm{NMR}\left(400 \mathrm{MHz}, \mathrm{CDCl}_{3}\right) \mathbf{S 6}$ 


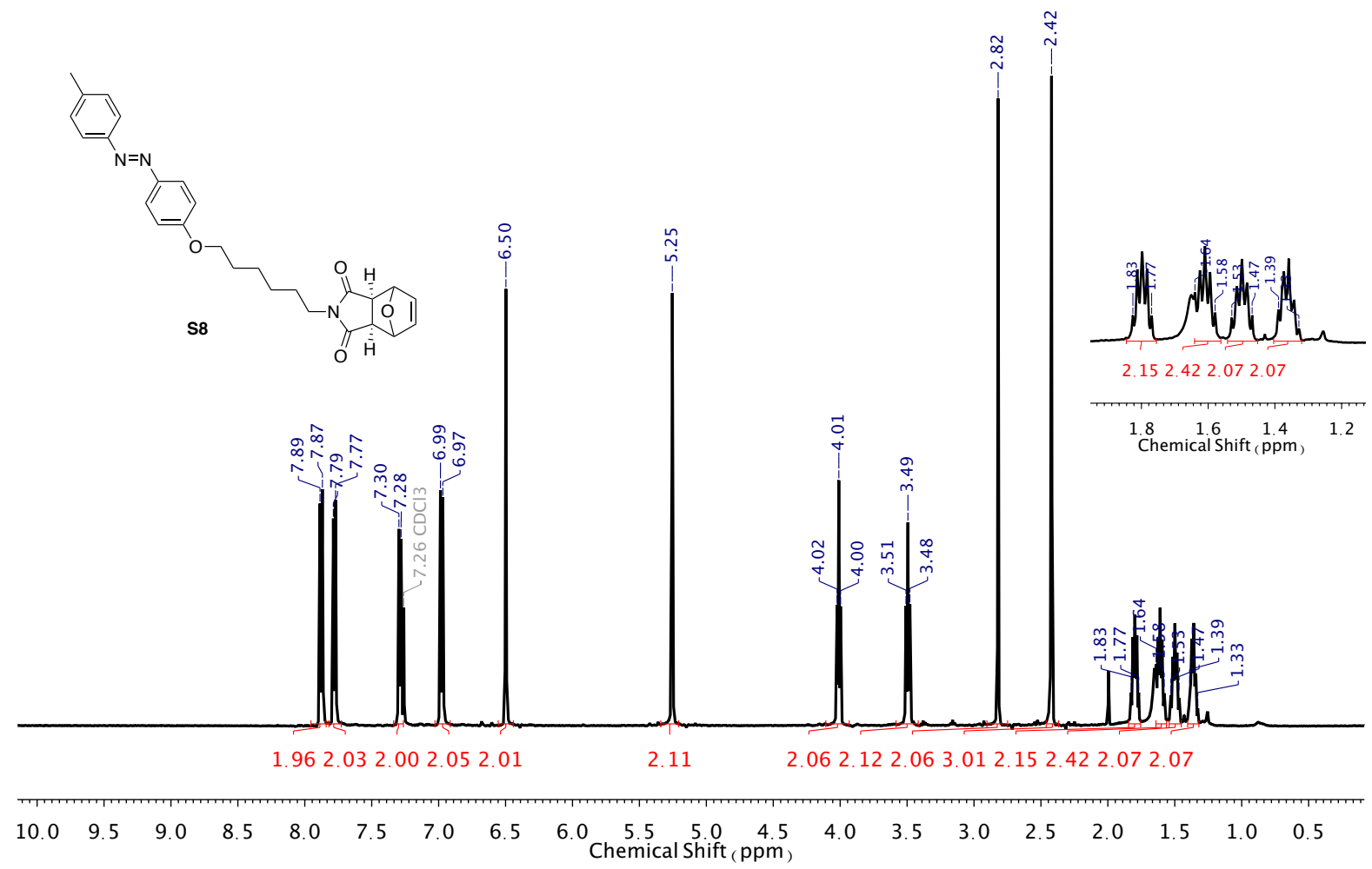

Figure S19. ${ }^{1} \mathrm{H}$ NMR (400 MHz, $\left.\mathrm{CDCl}_{3}\right) \mathbf{S 8}$

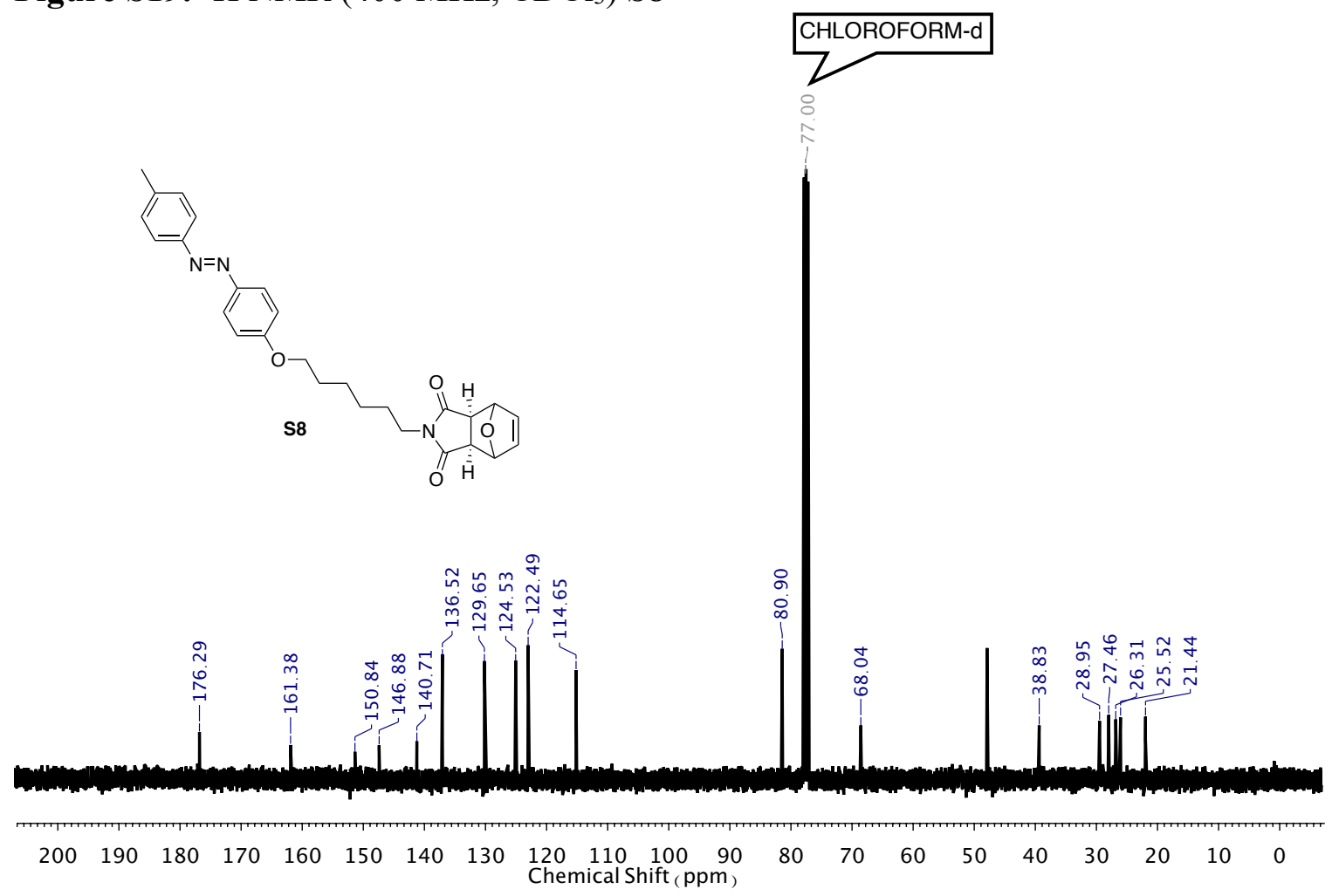

Figure S20. ${ }^{13} \mathrm{C}$ NMR (100 MHz, $\left.\mathrm{CDCl}_{3}\right) \mathbf{S 8}$ 


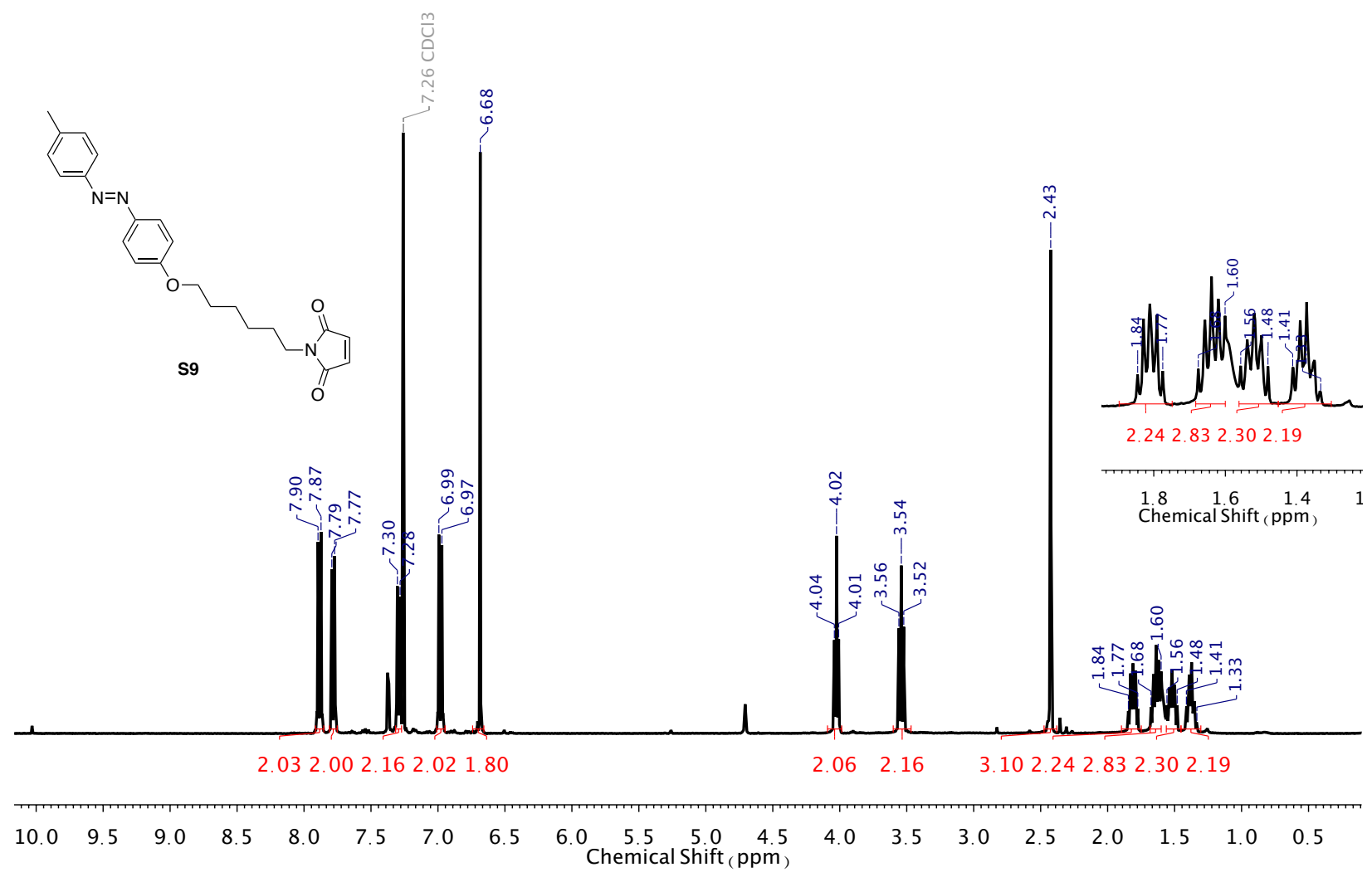

Figure S21. ${ }^{1} \mathrm{H} \mathrm{NMR}\left(400 \mathrm{MHz}, \mathrm{CDCl}_{3}\right) \mathbf{S 9}$

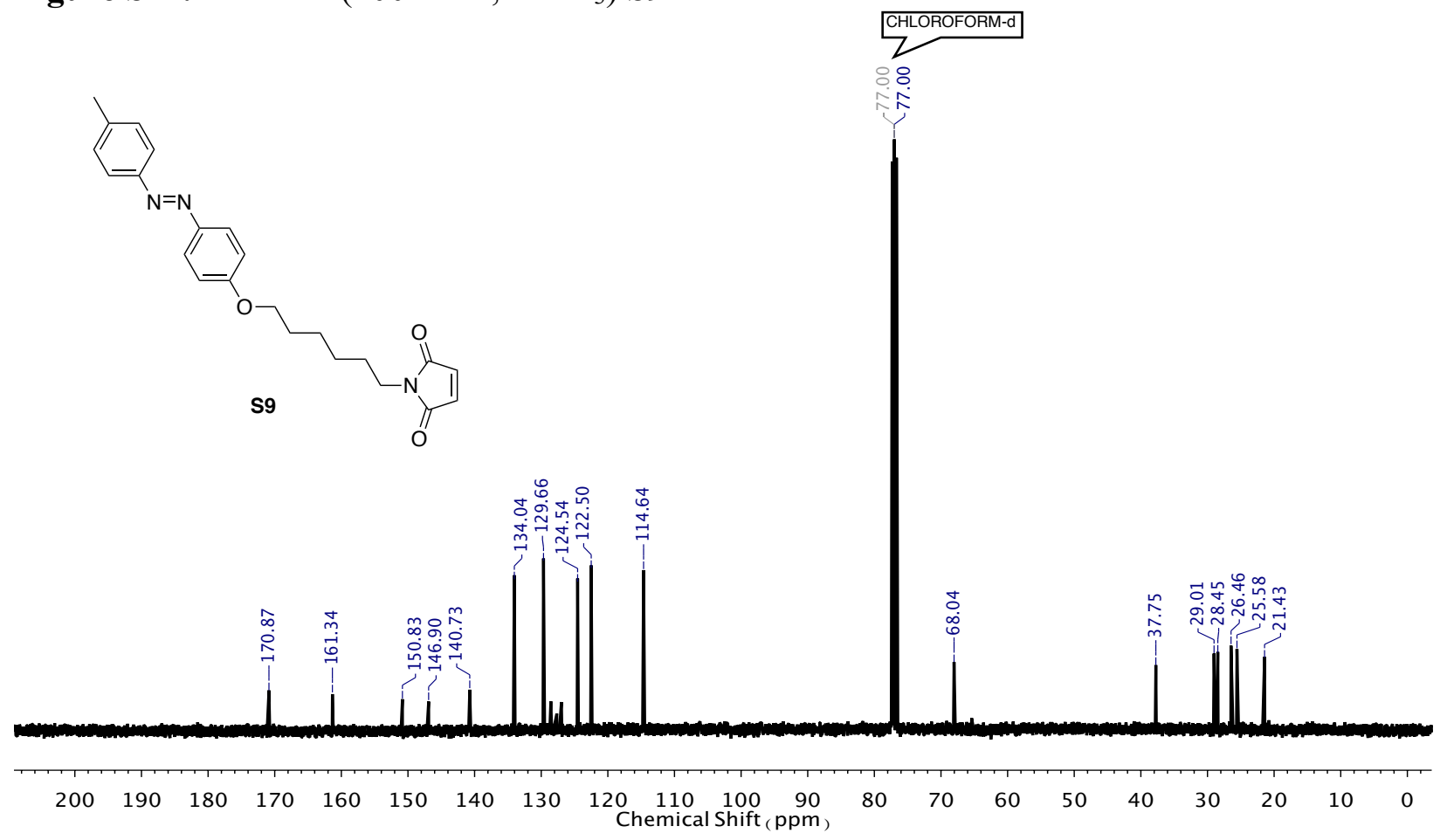

Figure S22. ${ }^{13} \mathrm{C}$ NMR (100 MHz, $\left.\mathrm{CDCl}_{3}\right) \mathbf{S 9}$ 


\section{Synthesis of Polymers}

\section{General Synthesis of NBD-PLA:}

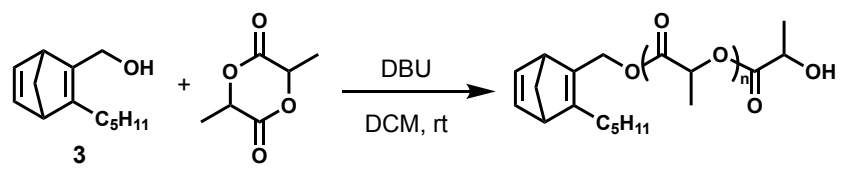

A $20-\mathrm{mL}$ scintillation vial equipped with a magnetic stir bar was flame dried under three argon and evacuation cycles before transfer into a glovebox. $D, L$-lactide (205-360 eq.) was added and dissolved in anhydrous DCM (5 mL/g $D, L$-lactide). 3 (1 eq.) was added to the vial along with a 1.5 M THF solution of 1,8-biazabicyclo[5.4.0]undec-7-ene (DBU) (1 eq.). The polymerization was allowed to stir at rt until desired conversion (4-12 min) and quenched with benzoic acid (5 eq.). Monomer conversion was estimated by ${ }^{1} \mathrm{H}$ NMR in deuterated chloroform. The DCM was removed under reduced pressure and the crude mixture was re-dissolved in a minimal amount of DCM. The mixture was subsequently precipitated into cold methanol before collecting the solid via vacuum filtration. The solid was re-dissolved in DCM, and the precipitation process was repeated twice. The obtained white solid was dried under reduced pressure.

\section{Synthesis of P1:}

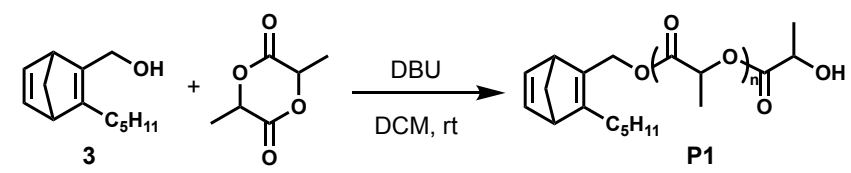

Followed general synthesis of NBD-PLA using 360 eq. $D, L$-lactide and 1.7 eq. DBU. Conversion $61 \%, M_{\mathrm{n} \text { (theoretical) }}=32,000 \mathrm{~g} / \mathrm{mol}, M_{\mathrm{n}(\mathrm{NMR})}=36,000 \mathrm{~g} / \mathrm{mol}, M_{\mathrm{n}(\mathrm{SEC})}=42,000 \mathrm{~g} / \mathrm{mol}, \emptyset=1.04$. 


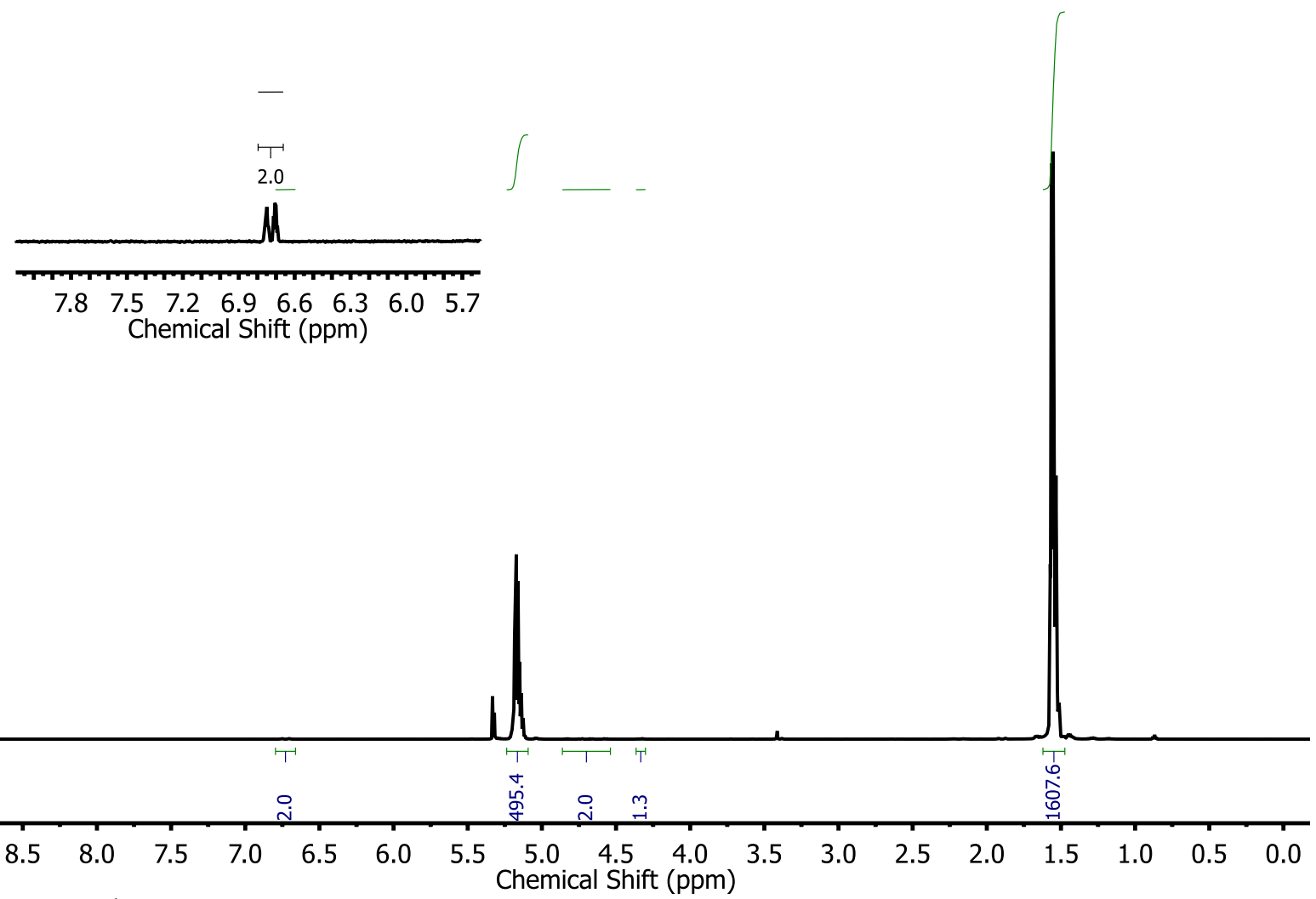

Figure S23. ${ }^{1} \mathrm{H}$ NMR of spectrum of $\mathbf{P 1}$ in $\mathrm{CD}_{2} \mathrm{Cl}_{2}$ showing the presence of expected chain-end resonances.

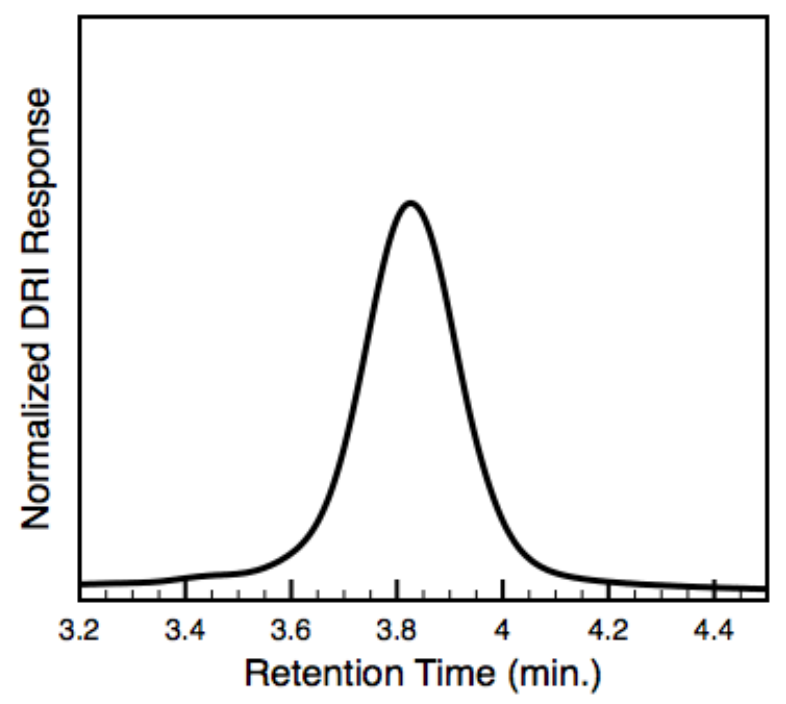

Figure S24. SEC-RI trace of P1. 


\section{Synthesis of S-P1:}
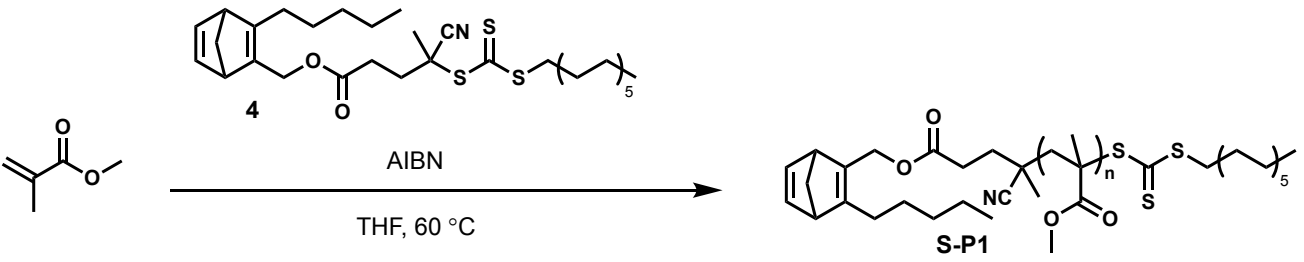

Methyl methacrylate (MMA, $825 \mu \mathrm{L}, 7.75 \mathrm{mmol}, 125$ eq.) was dissolved in $0.825 \mathrm{~mL}$ of THF in a 1-dram vial equipped with a magnetic stir bar and septum cap. Following this, azobisisobutyronitrile (AIBN, $2 \mathrm{mg}, 0.012 \mathrm{mmol}, 0.2$ eq.) and 4 (36 mg, $0.062 \mathrm{mmol}, 1$ eq.) were added and the resulting reaction mixture was degassed by argon bubbling for $10 \mathrm{~min}$. The polymerization proceeded at $60{ }^{\circ} \mathrm{C}$ for $7 \mathrm{~h}$. Monomer conversion was estimated by ${ }^{1} \mathrm{H}$ NMR in deuterated chloroform and determined to be $50 \%$. THF was removed under reduced pressure, and the crude mixture was re-dissolved in a minimal amount of DCM. The mixture was precipitated into cold methanol before collecting the solid via vacuum filtration. The solid was re-dissolved in $\mathrm{DCM}$, and the precipitation process was repeated a total of 3 times. ${ }^{1} \mathrm{H}$ NMR analysis indicated successful incorporation of the NBD chain-end functionality. Conversion $50 \%, M_{\mathrm{n} \text { (theoretical) }}=6,900$ $\mathrm{g} / \mathrm{mol}, M_{\mathrm{n}(\mathrm{NMR})}=8,700 \mathrm{~g} / \mathrm{mol}, M_{\mathrm{n}(\mathrm{SEC})}=7,700 \mathrm{~g} / \mathrm{mol}, \oslash=1.14$.

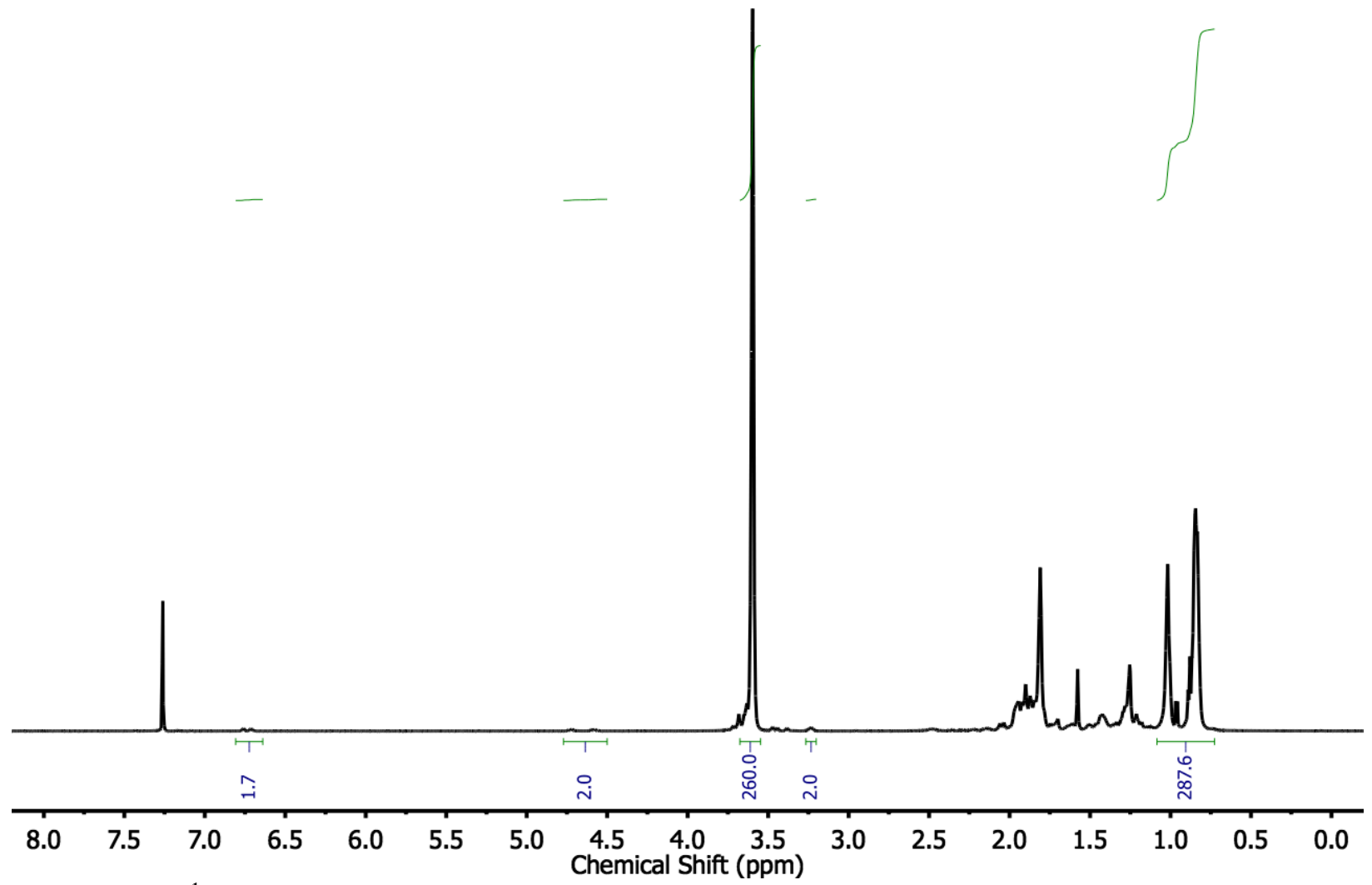

Figure S25. ${ }^{1} \mathrm{H}$ NMR of spectrum of $\mathbf{S}-\mathbf{P 1}$ in $\mathrm{CDCl}_{3}$ showing the presence of expected chain-end resonances. 


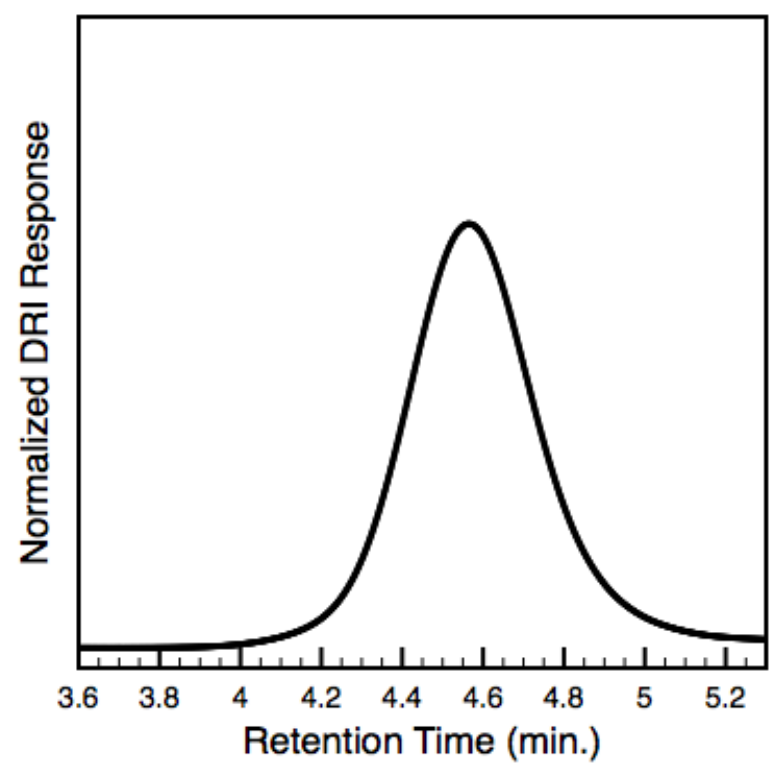

Figure S26. SEC-RI trace of S-P1.

\section{Synthesis of S-P2}

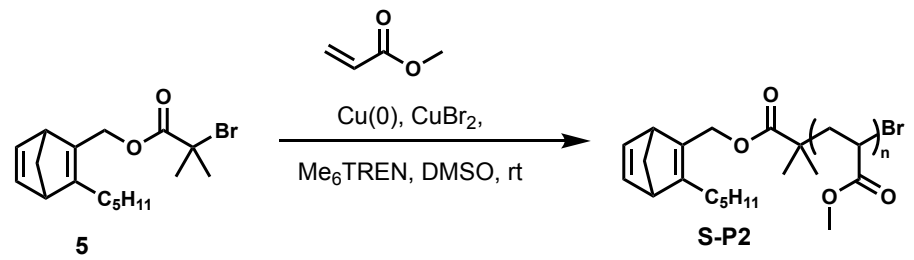

Copper(II) bromide (CuBr2, $1.1 \mathrm{mg}, 0.05 \mathrm{mmol}, 0.50$ eq.) and tris[2-(dimethylamino)ethyl]amine (Me6TREN) $(4.8 \mu \mathrm{L}, 0.018 \mathrm{mmol}, 0.18$ eq.) were added to a 1 -dram vial equipped with a septum sealed cap then dissolved in DMSO $(0.50 \mathrm{~mL})$ with sonication for $10 \mathrm{~min}$. Methyl acrylate $(0.45$ $\mathrm{mL}, 5.0 \mathrm{mmol}, 50$ eq.) was added to the catalyst mixture. Compound 5 ( $34.1 \mathrm{mg}, 0.1 \mathrm{mmol}, 1.0$ eq.) was added subsequently. Copper wire $(3 \mathrm{~cm}, 22 \mathrm{AWG})$ was activated by soaking in concentrated $\mathrm{HCl}$ for $7 \mathrm{~min}$, then wrapped around a magnetic stir bar and placed in the vial. The solution was degassed by argon bubbling for $10 \mathrm{~min}$ while keeping the stir bar suspended above the solution using a magnet. The polymerization was stirred at $\mathrm{rt}$ for $10 \mathrm{~min}$. The crude polymer was precipitated once in cold hexanes. The precipitated solid was collected, re-dissolved in dichloromethane and precipitated once in cold methanol $\mathrm{MeOH}$. The precipitated solid was collected by vacuum filtration and dried under reduced pressure. Conversion $60 \%, M_{n \text { (theoretical) }}=$ $2,900 \mathrm{~g} / \mathrm{mol}, M_{n(\mathrm{NMR})}=2,100 \mathrm{~g} / \mathrm{mol}, M_{n(\mathrm{SEC})}=2,100 \mathrm{~g} / \mathrm{mol}, Ð=1.31$. 


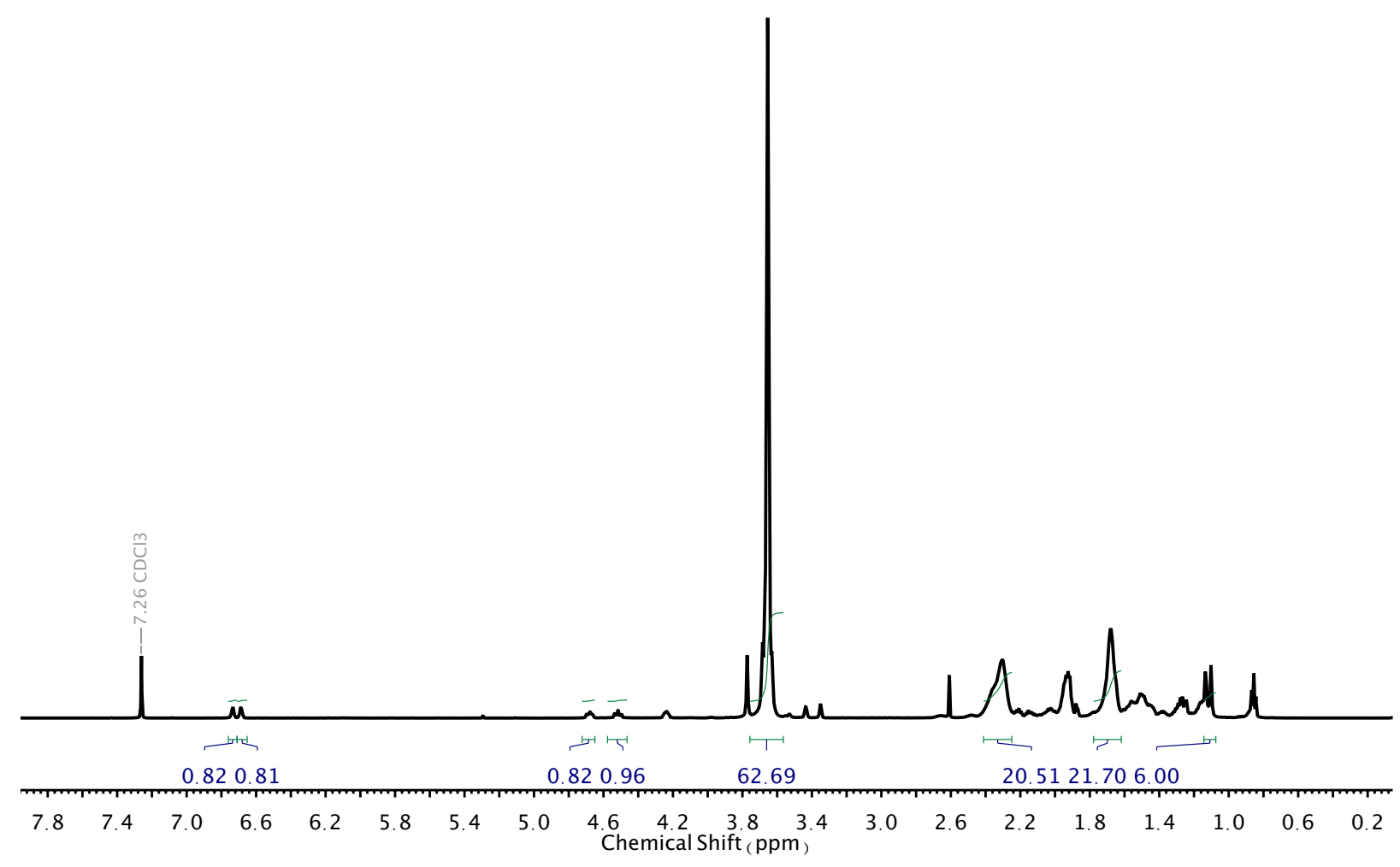

Figure S27. ${ }^{1} \mathrm{H}$ NMR of spectrum of $\mathbf{S - P 2}$ in $\mathrm{CDCl}_{3}$ showing presence of expected chain-end resonances.

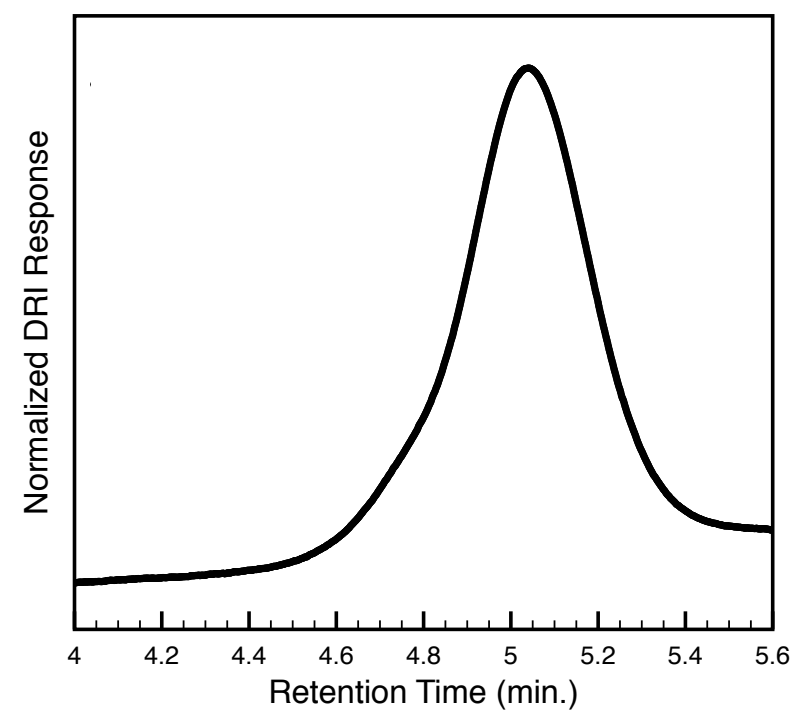

Figure S28. SEC-RI trace of S-P2. 


\section{Synthesis of S-P3}

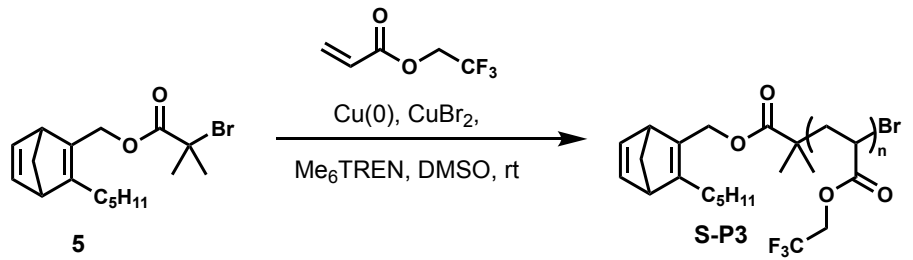

$\mathrm{CuBr}_{2}$ (1.1 mg, $0.005 \mathrm{mmol}, 0.5$ eq.) and Me6-Tren ( $4.8 \mu \mathrm{L}, 0.018 \mathrm{mmol}, 0.18$ eq.) were added to a $20-\mathrm{mL}$ vial equipped with a septum sealed cap and dissolved in DMSO $(2.50 \mathrm{~mL})$ via sonication for $10 \mathrm{~min}$. Trifluoroethyl acrylate $(2.54 \mathrm{~mL}, 10.0 \mathrm{mmol}, 100 \mathrm{eq}$.) was added to the catalyst mixture. Compound 5 ( $34.1 \mathrm{mg}, 0.1 \mathrm{mmol}, 1$ eq.) was added subsequently. Copper wire $(3 \mathrm{~cm}, 22$ AWG) was activated by soaking in concentrated $\mathrm{HCl}$ for 7 min then wrapped around a magnetic stir bar and placed in the vial. The solution was degassed by argon bubbling for 10 min while keeping the stir bar suspended above the solution with a magnet. The polymerization was stirred at $\mathrm{rt}$ for $20 \mathrm{~min}$. The crude polymer was passed through basic alumina to remove copper before dialysis in DCM for 2 days. The dialyzed polymer solution was collected by vacuum filtration and dried under reduced pressure. Conversion $20 \%, M_{n \text { (theoretical) }}=3,400 \mathrm{~g} / \mathrm{mol}, M_{n \text { (NMR) }}=3,100$ $\mathrm{g} / \mathrm{mol}, M_{n}(\mathrm{SEC})=1,800 \mathrm{~g} / \mathrm{mol}, \nexists=1.32$.

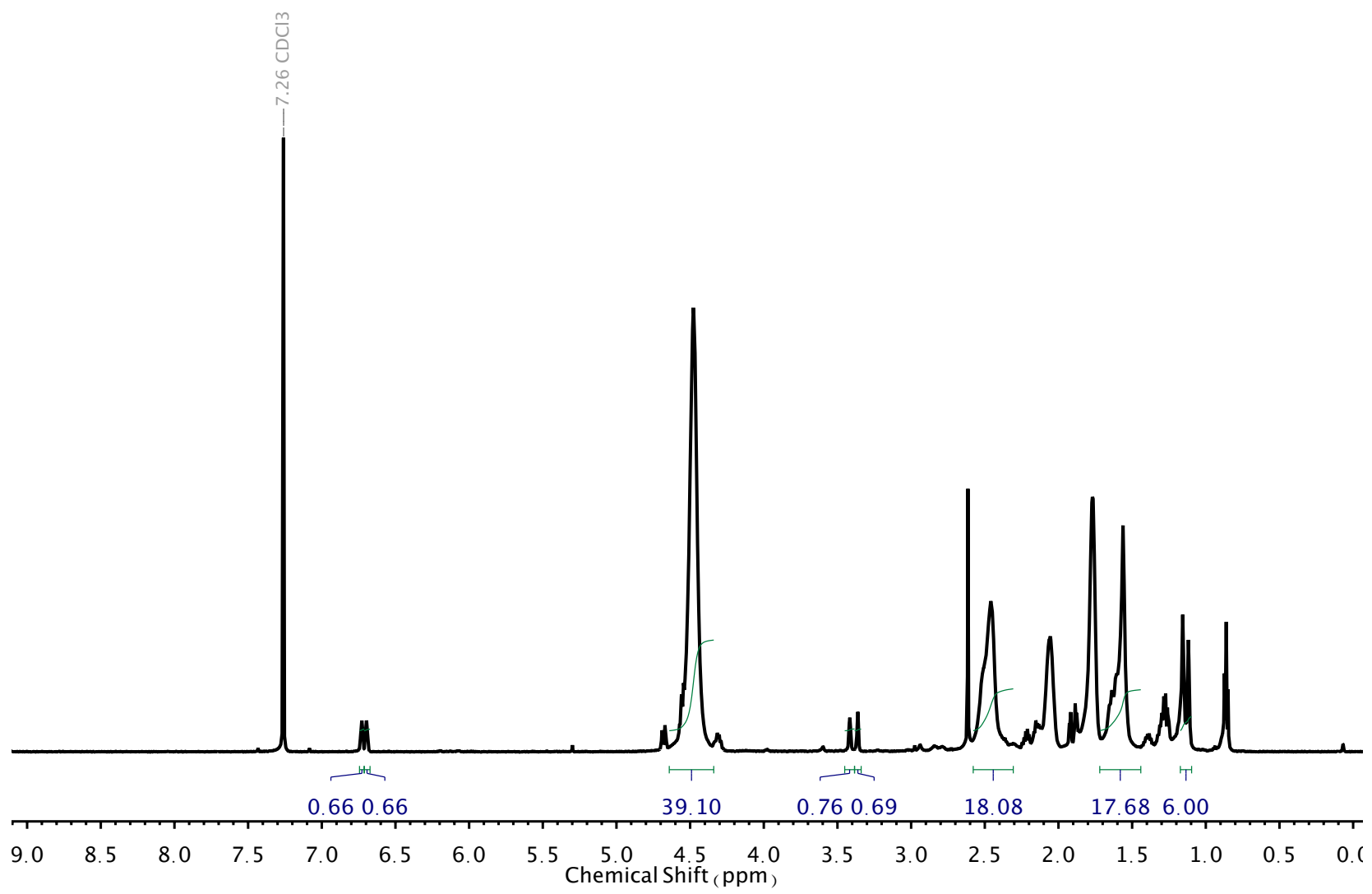

Figure S29. ${ }^{1} \mathrm{H}$ NMR of spectrum of $\mathbf{S - P 3}$ in $\mathrm{CDCl}_{3}$ showing presence of expected chain-end resonances. 


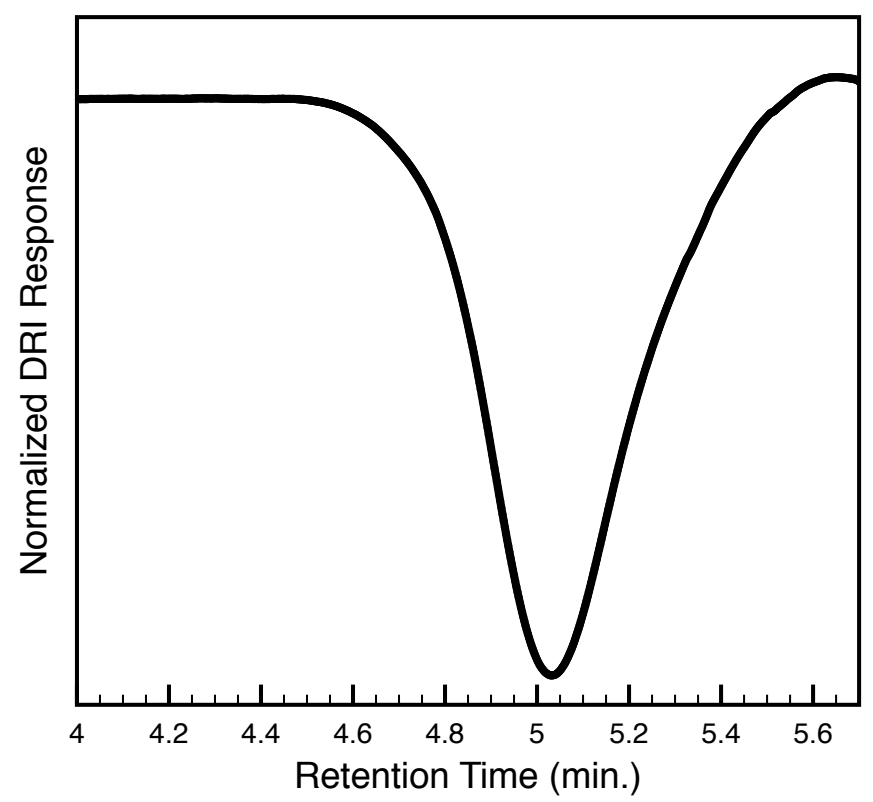

Figure S30. SEC-RI trace of S-P3.

Synthesis of P2:

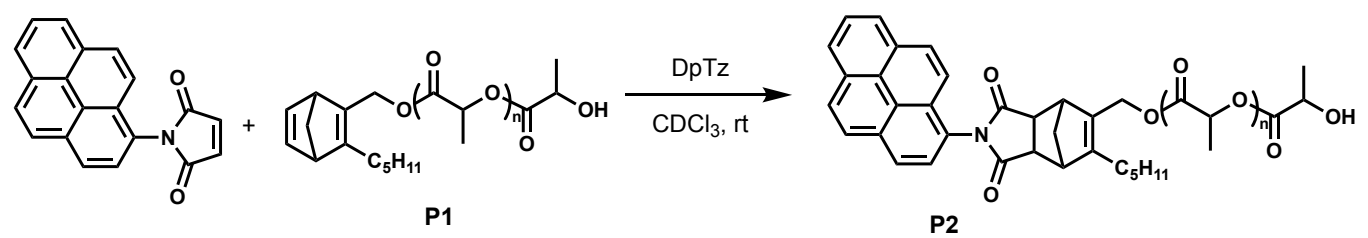

P1 (79 mg, 0.002 mmol, 1 eq.), 3,6-di-2-pyridyl-1,2,4,5-tetrazine (DpTz) (1.3 mg, $0.005 \mathrm{mmol}$, 2.4 eq.) and a solution of $N$-(1-pyrenyl)maleimide in $\mathrm{CDCl}_{3}(4 \mathrm{mg} / \mathrm{mL}, 0.2 \mathrm{~mL}, 0.003 \mathrm{mmol}, 1.2$ eq.)) were added to a 1-dram vial equipped with a septum cap. The vial was placed on a shaker at rt for $4 \mathrm{~h}$, before adding approximately $1 \mathrm{mg}$ of norbornene to quench the excess DpTz. The reaction was left to shake at $\mathrm{rt}$ for an additional $20 \mathrm{~h}$. The crude reaction mixture was precipitated into cold methanol and centrifuged to collect the desired product. The supernatant was discarded, and the resulting solid was collected and re-dissolved in a minimal amount of DCM . The precipitation process was repeated 3 times before drying the obtained polymer under reduced pressure. ${ }^{1} \mathrm{H}$ NMR of the purified polymer suggests near quantitative conversion to P2. $M_{\mathrm{n}(\mathrm{NMR})}=36,000 \mathrm{~g} / \mathrm{mol}, M_{\mathrm{n}(\mathrm{SEC})}=42,000 \mathrm{~g} / \mathrm{mol}, \emptyset=1.04$. 

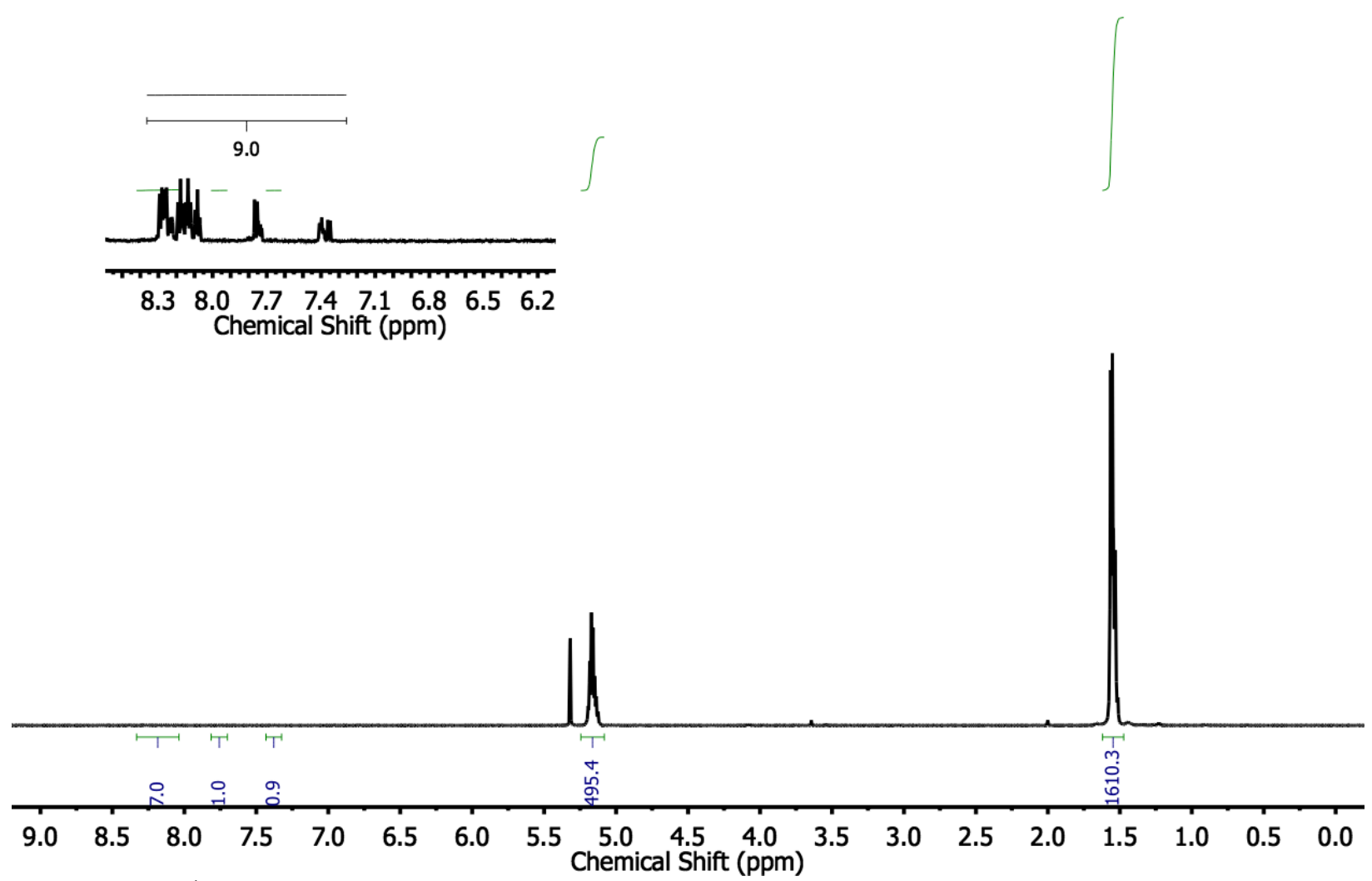

Figure S31. ${ }^{1} \mathrm{H}$ NMR of spectrum of $\mathbf{P 2}$ in $\mathrm{CDCl}_{3}$ showing presence of expected chain-end resonances corresponding to pyrene following functionalization.

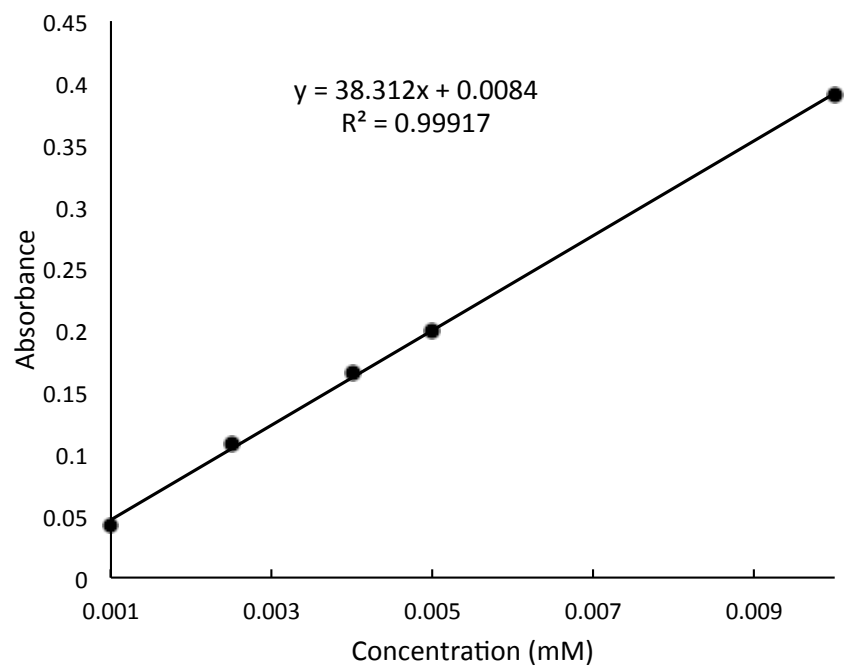

Figure S32. Calibration curve prepared using serial dilutions of $N$-(1-pyrenyl)maleimide in DCM based on local maximum at $276 \mathrm{~nm}$. 


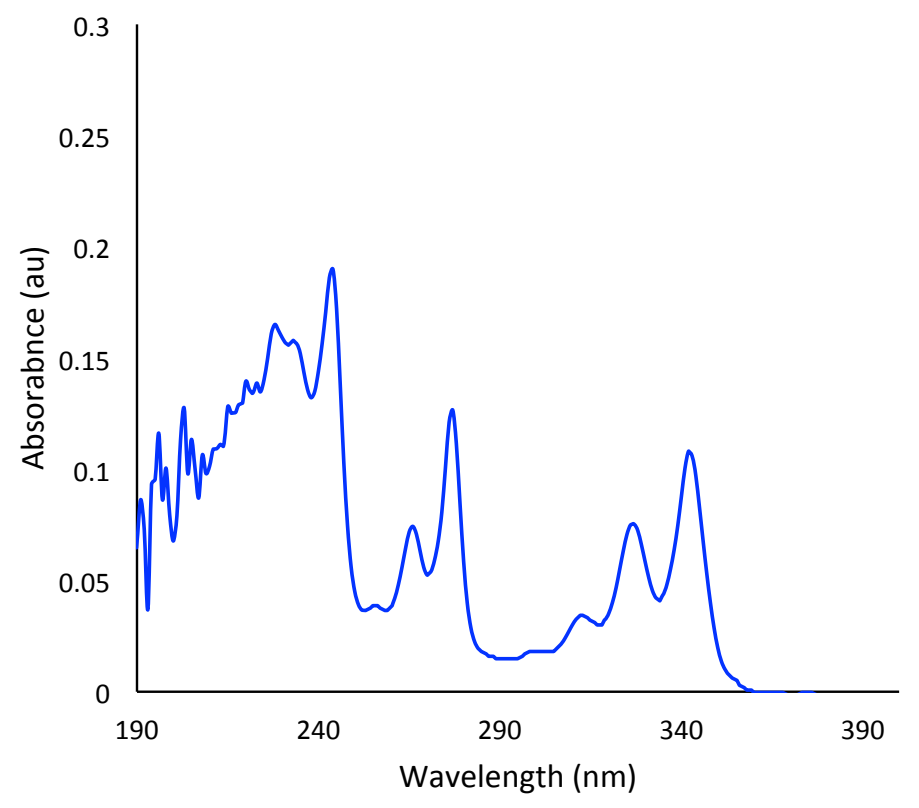

Figure S33. Absorbance spectrum of a $0.003 \mathrm{mM}$ solution of P2 $\left(M_{\mathrm{n}(\mathrm{NMR})}=36,000 \mathrm{~g} / \mathrm{mol}\right)$ in DCM shows incorporation of pyrene functionality. Using the absorbance value for the local $276 \mathrm{~nm}$ maximum and the linear regression equation obtained from the calibration curve, the incorporation of pyrene is estimated to be 0.98 pyrenes/per chain. These results support the quantitative incorporation calculated by ${ }^{1} \mathrm{H}$ NMR.

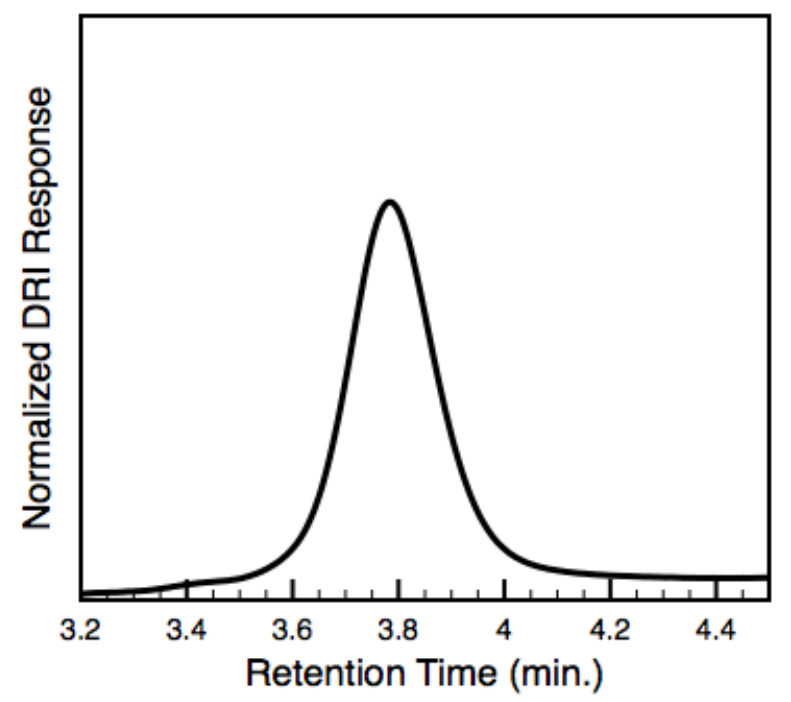

Figure S34. SEC-RI trace of $\mathbf{P 2}$ after functionalization showing no observable change in molar mass distribution. 


\section{Synthesis of S-P5:}
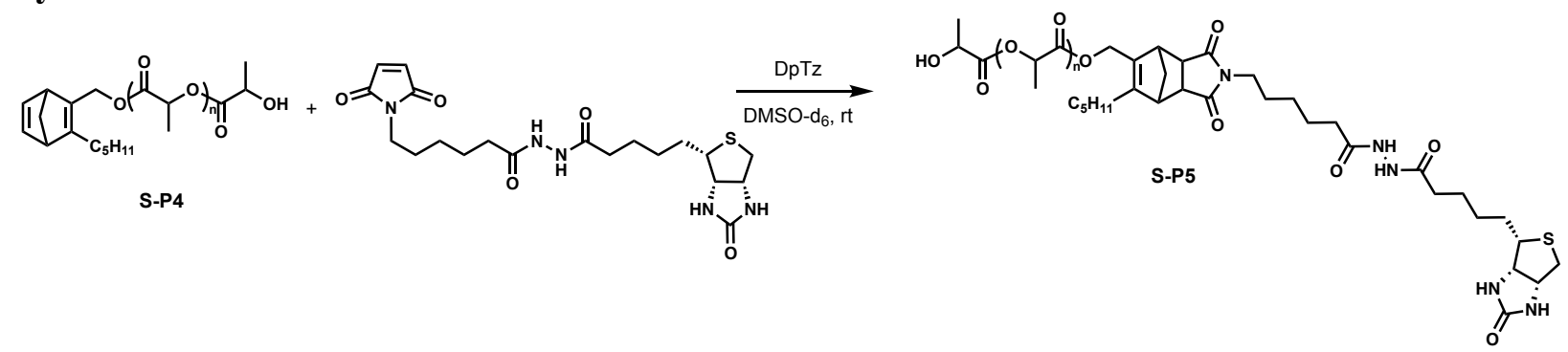

S-P4 $\left(70 \mathrm{mg}, 0.0037 \mathrm{mmol}, 1\right.$ eq., prepared following general procedure for NBD-PLA. $M_{\mathrm{n}(\mathrm{NMR})}=$ $\left.20,00 \mathrm{~g} / \mathrm{mol}, M_{\mathrm{n}(\mathrm{SEC})}=14,000 \mathrm{~g} / \mathrm{mol}, \emptyset=1.10\right)$, biotin-maleimide ( $3.7 \mathrm{mg}, 0.008 \mathrm{mmol}, 2.2$ eq.) and 3,6-di-2-pyridyl-1,2,4,5-tetrazine (DpTz) $(2.2 \mathrm{mg}, 0.009 \mathrm{mmol}, 2.5 \mathrm{eq}$.) were added to a 1dram vial equipped with septum cap and dissolved in DMSO- $d_{6}(0.37 \mathrm{~mL})$. The vial was placed on a shaker at $\mathrm{rt}$ for $8 \mathrm{~h}$. The crude reaction mixture was precipitated into isopropanol and filtered to collect the desired product. The resulting solid was collected, and re-dissolved in a minimal amount of DCM before repeating the precipitation process. This process was repeated 6 times before drying the obtained polymer under reduced pressure. ${ }^{1} \mathrm{H}$ NMR of the purified polymer suggests near quantitative conversion to S-P5. $M_{\mathrm{n}(\mathrm{NMR})}=20,000 \mathrm{~g} / \mathrm{mol}, M_{\mathrm{n}(\mathrm{SEC})}=14,000$ $\mathrm{g} / \mathrm{mol}, \emptyset=1.12$.
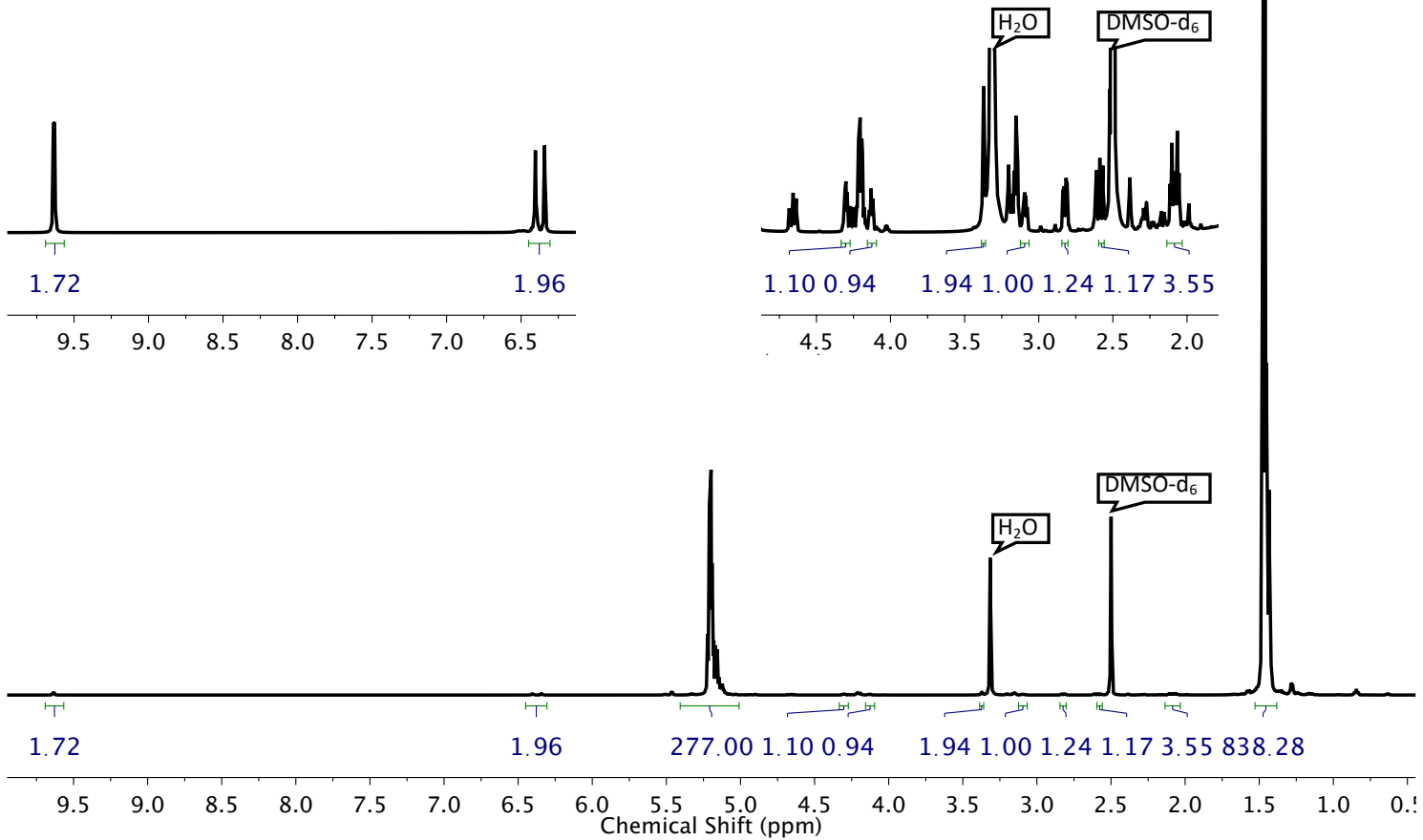

Figure S35. ${ }^{1} \mathrm{H}$ NMR of spectrum of S-P5 in DMSO- $\mathrm{d}_{6}$ showing presence of expected chain-end resonances corresponding to biotin following functionalization. 


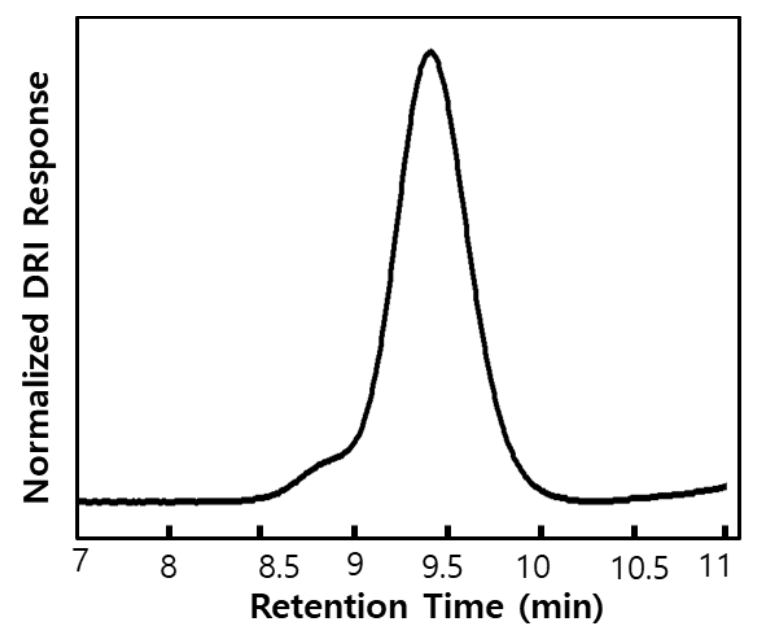

Figure S36. SEC-RI trace of S-P5.

\section{Synthesis of P3:}

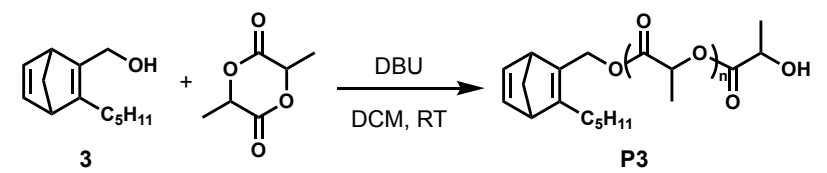

Followed general synthesis of NBD-PLA using 210 eq. $D, L$-lactide. Conversion $51 \%, M_{\mathrm{n} \text { (theoretical) }}=15,700 \mathrm{~g} / \mathrm{mol}, M_{\mathrm{n}(\mathrm{NMR})}=19,700 \mathrm{~g} / \mathrm{mol}, M_{\mathrm{n}(\mathrm{SEC})}=21,800 \mathrm{~g} / \mathrm{mol}, D=1.08$.

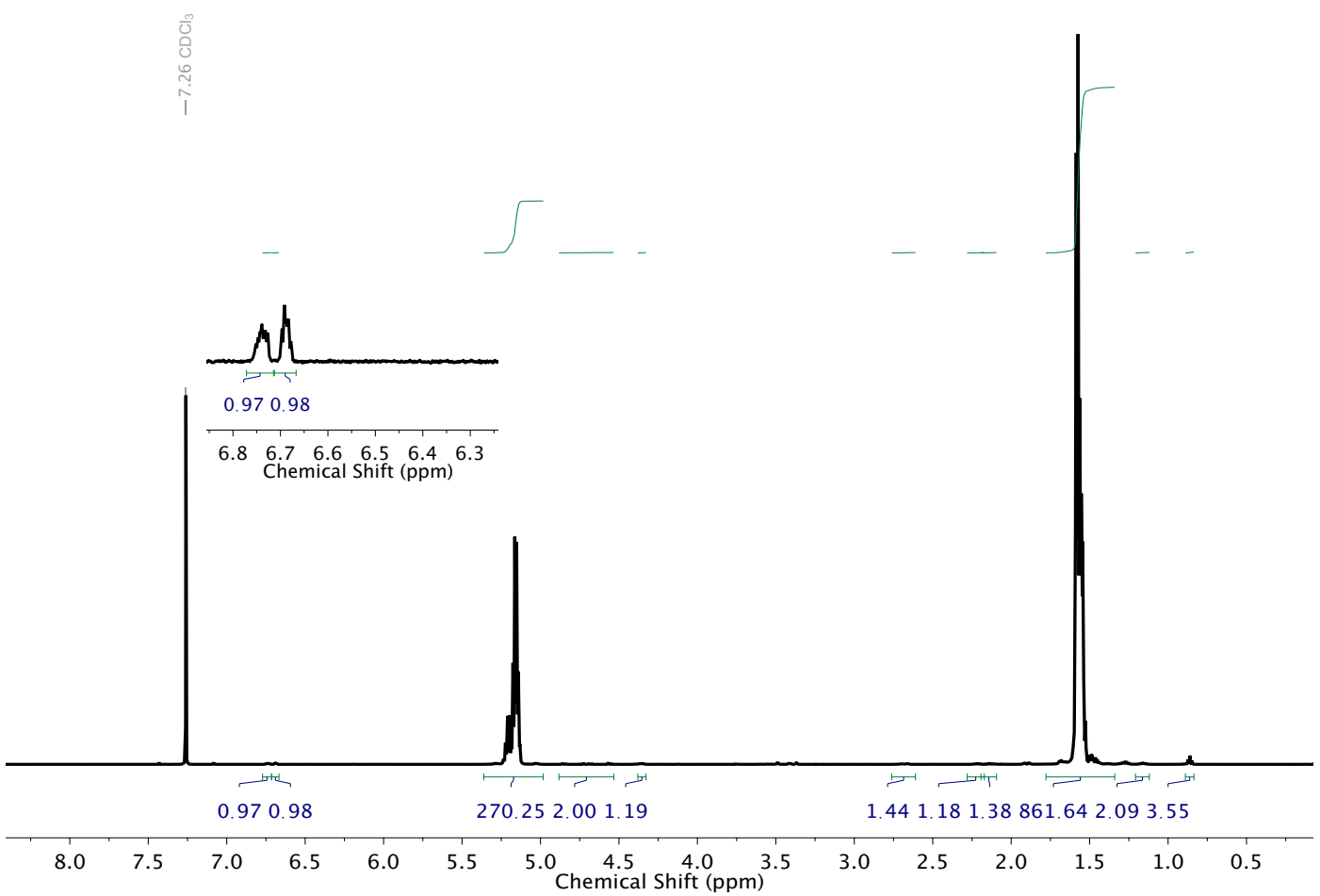

Figure S37. ${ }^{1} \mathrm{H}$ NMR of spectrum of $\mathbf{P 3}$ in $\mathrm{CDCl}_{3}$ showing presence of expected chain-end resonances 


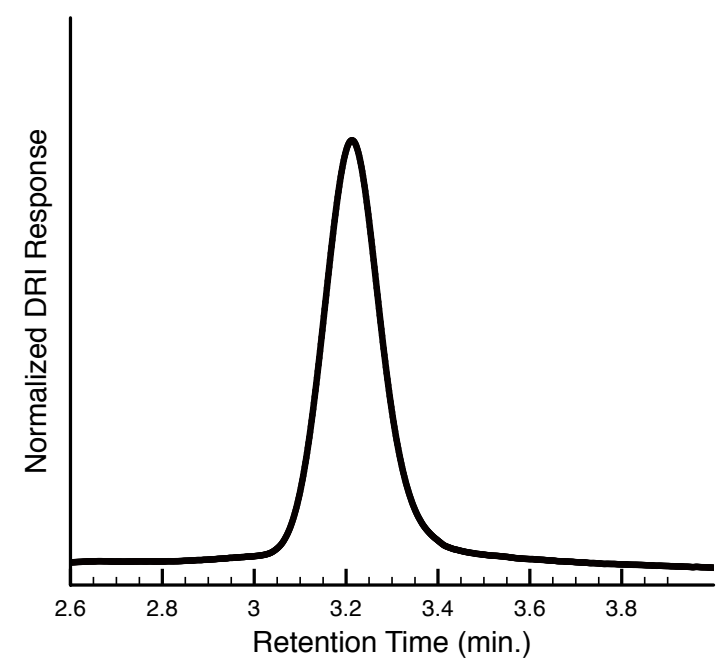

Figure S38. SEC-RI trace of P3.

\section{Synthesis of S-P6}

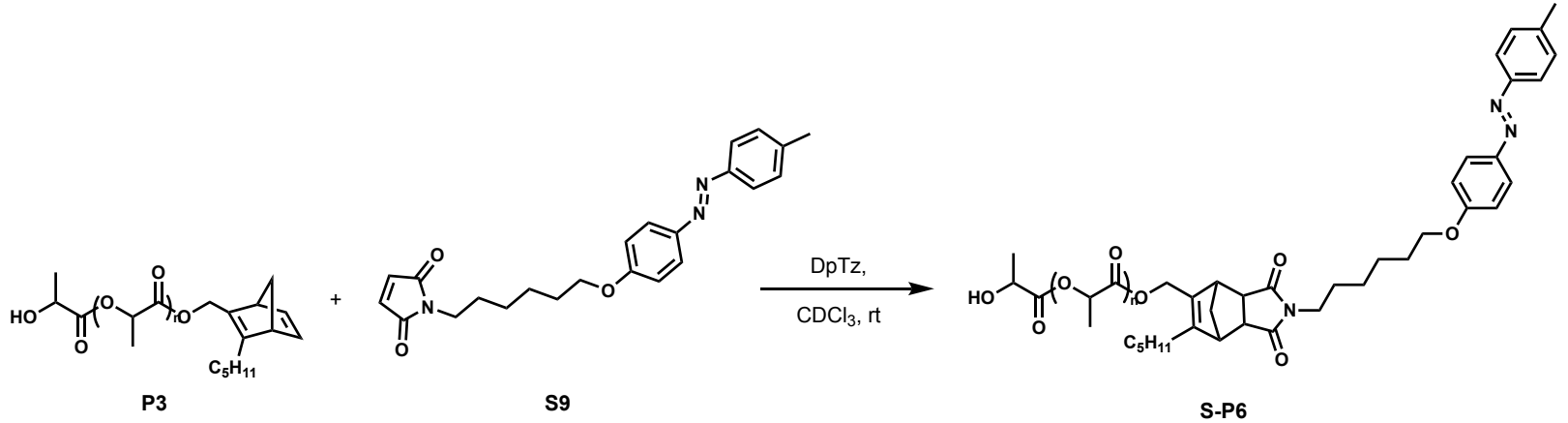

P3 (100 mg, $0.0051 \mathrm{mmol}, 1$ eq.), 3,6-di-2-pyridyl-1,2,4,5-tetrazine (DpTz) (2.8 mg, $0.012 \mathrm{mmol}$, 2.3 eq.) and $\mathbf{S 9}$ (2.4 mg, $0.0061 \mathrm{mmol}, 1.2$ eq.) were added to a 1-dram vial equipped with a septum cap and dissolved in $0.8 \mathrm{~mL} \mathrm{CDCl}_{3}$. The solution was stirred at $\mathrm{rt}$ for $32 \mathrm{~h}$, before adding approximately $1 \mathrm{mg}$ of norbornene to quench the excess DpTz. The crude reaction mixture was precipitated into $10 \mathrm{~mL}$ methanol and centrifuged to collect the desired product. The supernatant was discarded, and the resulting solid was collected, and re-dissolved in a minimal amount of DCM before repeating the precipitation process. This process was repeated a total of 3 times before drying the obtained polymer under reduced pressure to afford $38 \mathrm{mg}(0.0019 \mathrm{mmol}, 37 \%)$ of SP6. ${ }^{1} \mathrm{H}$ NMR of the purified polymer suggests near quantitative conversion to $\mathbf{S}-\mathbf{P 6}$. $M_{\mathrm{n}(\mathrm{NMR})}=$ $20,000 \mathrm{~g} / \mathrm{mol}, M_{\mathrm{n}(\mathrm{SEC})}=26,000 \mathrm{~g} / \mathrm{mol}, \emptyset=1.08$. 

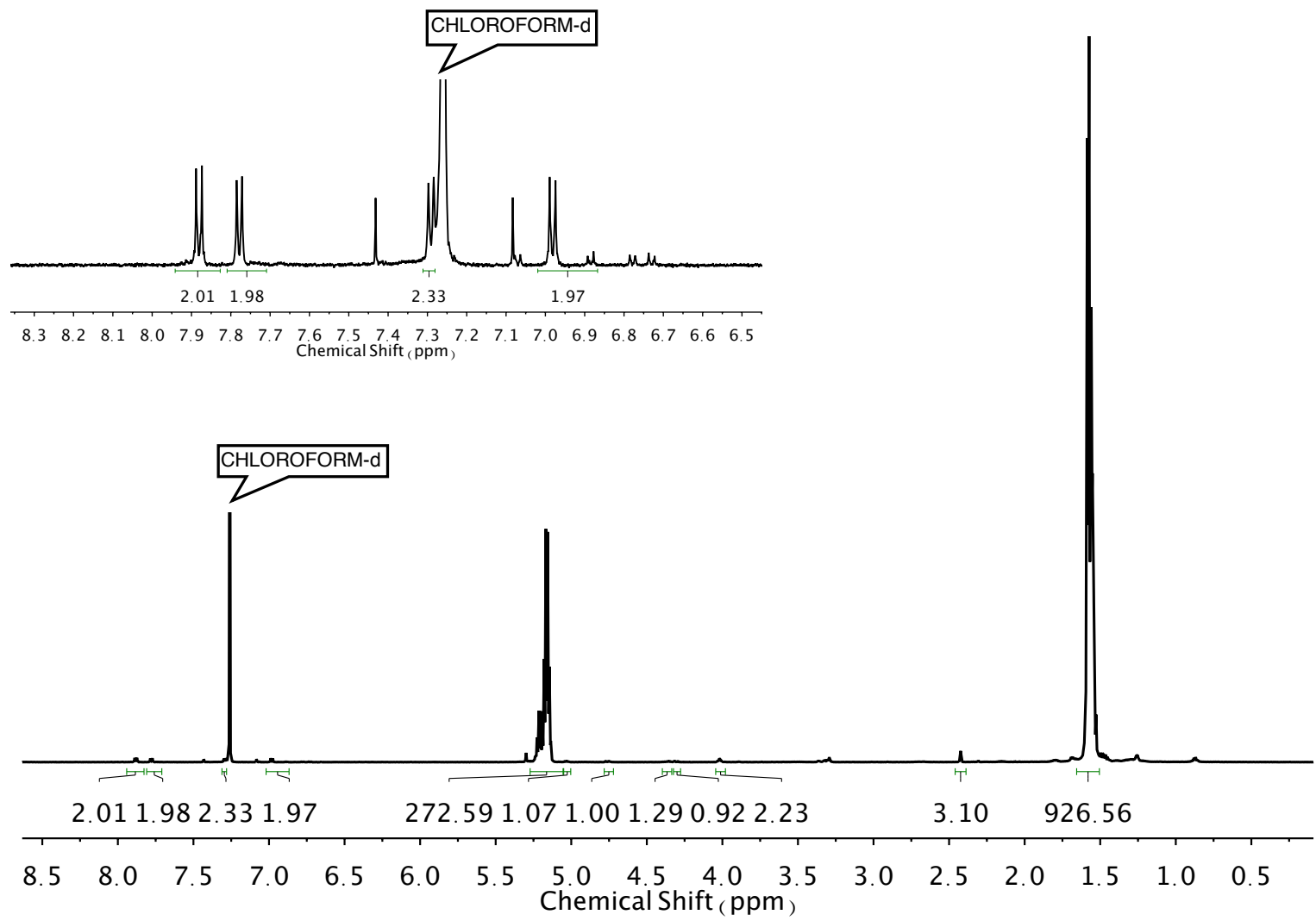

Figure S39. ${ }^{1} \mathrm{H}$ NMR of spectrum of $\mathbf{S - P 6}$ in $\mathrm{CDCl}_{3}$ showing presence of expected chain-end resonances corresponding to azobenzene following functionalization.

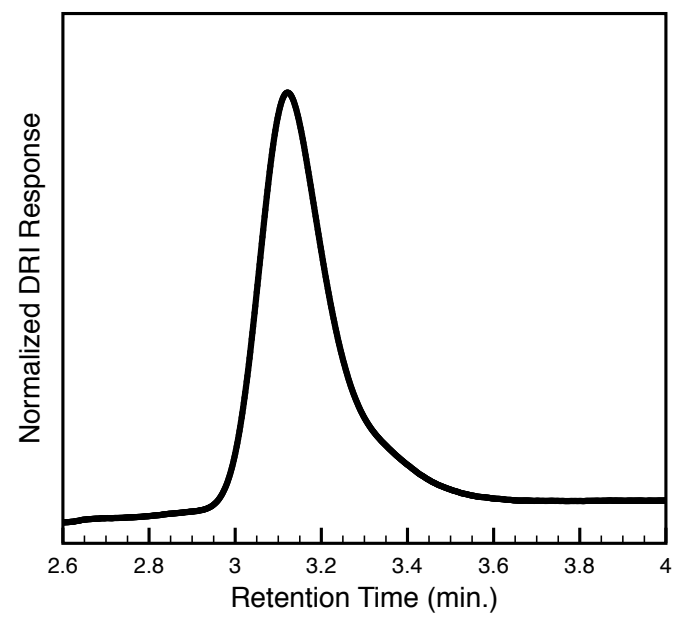

Figure S40. SEC-RI trace of S-P6 after functionalization showing no observable change in molar mass distribution. 


\section{Synthesis of SP-8:}

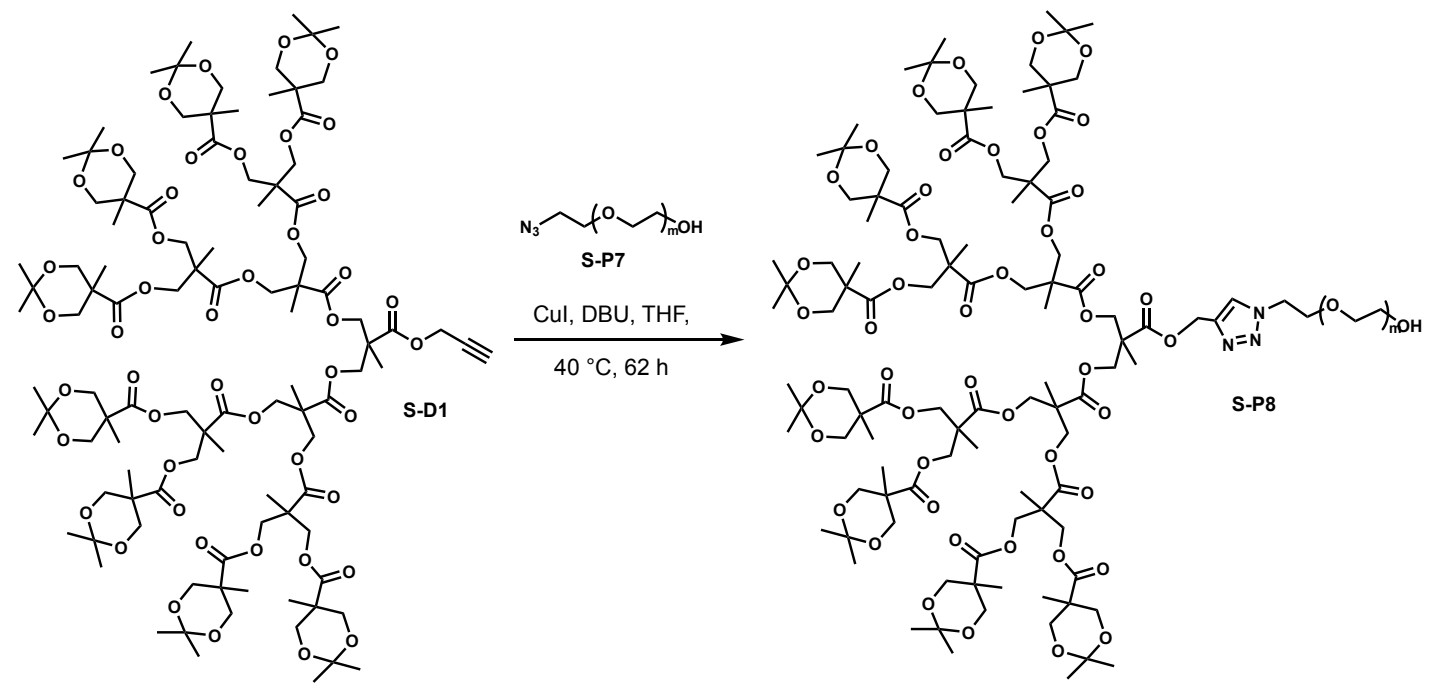

S-P7 (86 mg, 0.039 mmol, 1 eq., provided by Intezyne Technologies, $M_{\mathrm{n}(\mathrm{NMR})}=2,200$ $\left.\mathrm{g} / \mathrm{mol}, M_{\mathrm{n}(\mathrm{SEC})}=3,400 \mathrm{~g} / \mathrm{mol}, \emptyset=1.13\right)$, S-D1 (91 mg, $0.043 \mathrm{mmol}, 1.1$ eq., purchased from Polymer Factory) and $\mathrm{CuI}$ (1.5 mg, $0.008 \mathrm{mmol}, 0.2$ eq.) was added to a flame-dried, 1-dram vial under nitrogen and equipped with a stir bar. A solution of DBU (117 $\mu \mathrm{L}, 0.78 \mathrm{mmol}, 20$ eq.) and dry THF $(2 \mathrm{~mL})$ was transferred into the vial. The vial was sealed under nitrogen and heated at $40^{\circ} \mathrm{C}$ for $62 \mathrm{~h}$. The solution was brought to rt before passing through a silica plug and rinsing with $20 \mathrm{~mL}$ of MeOH:DCM (1:9). The solvent was removed, and the crude polymer was precipitated three times in $10 \mathrm{~mL}$ cold EtOH $\left(-78^{\circ} \mathrm{C}\right)$ to afford $84 \mathrm{mg}(0.019 \mathrm{mmol}, 48 \%)$ of $\mathbf{S}-\mathbf{P 8} . M_{\mathrm{n}(\mathrm{NMR})}=$ $4,500 \mathrm{~g} / \mathrm{mol}, M_{\mathrm{n}(\mathrm{SEC})}=4,600 \mathrm{~g} / \mathrm{mol}, \emptyset=1.12$.

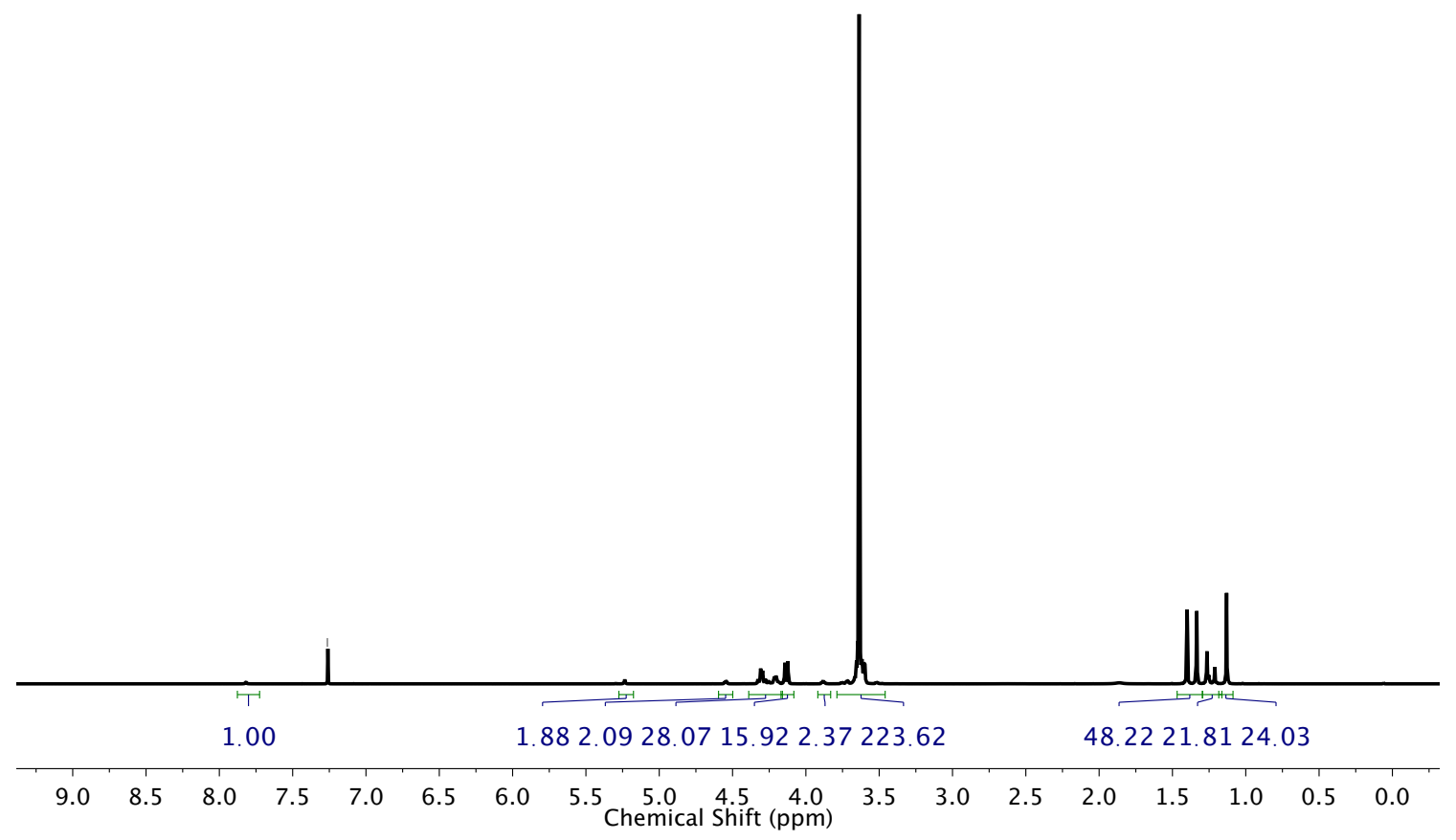

Figure S41. ${ }^{1} \mathrm{H}$ NMR of spectrum of $\mathbf{S - P 8}$ in $\mathrm{CDCl}_{3}$ showing presence of expected triazole and dendron resonances. 


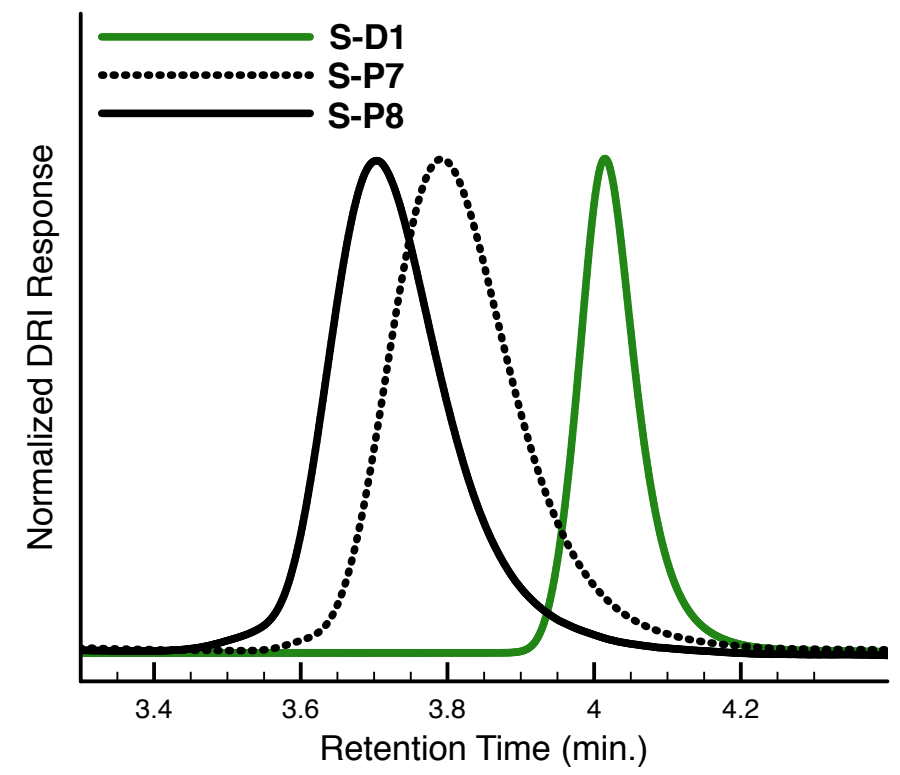

Figure S42. Overlay of SEC-RI traces of S-D1, S-P7 and S-P8 showing expected shift to higher molecular weight after $\mathrm{CuAAC}$.

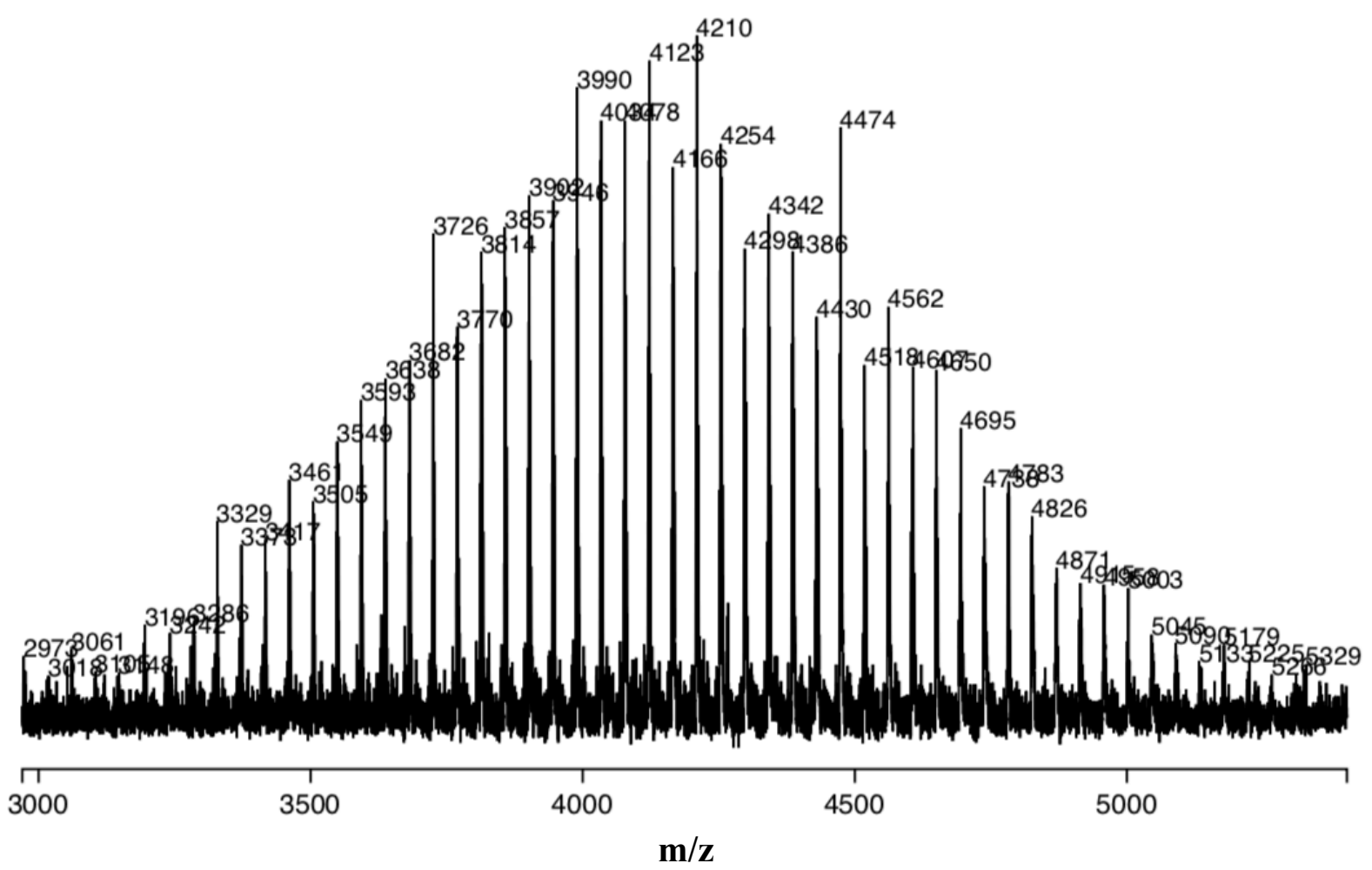

Figure S43. MALDI shows expected distribution of masses for S-P8. Calculated [DP $=28+$ $\mathrm{Na}]^{+}=3504$, observed 3505 . 


\section{Synthesis of P4:}

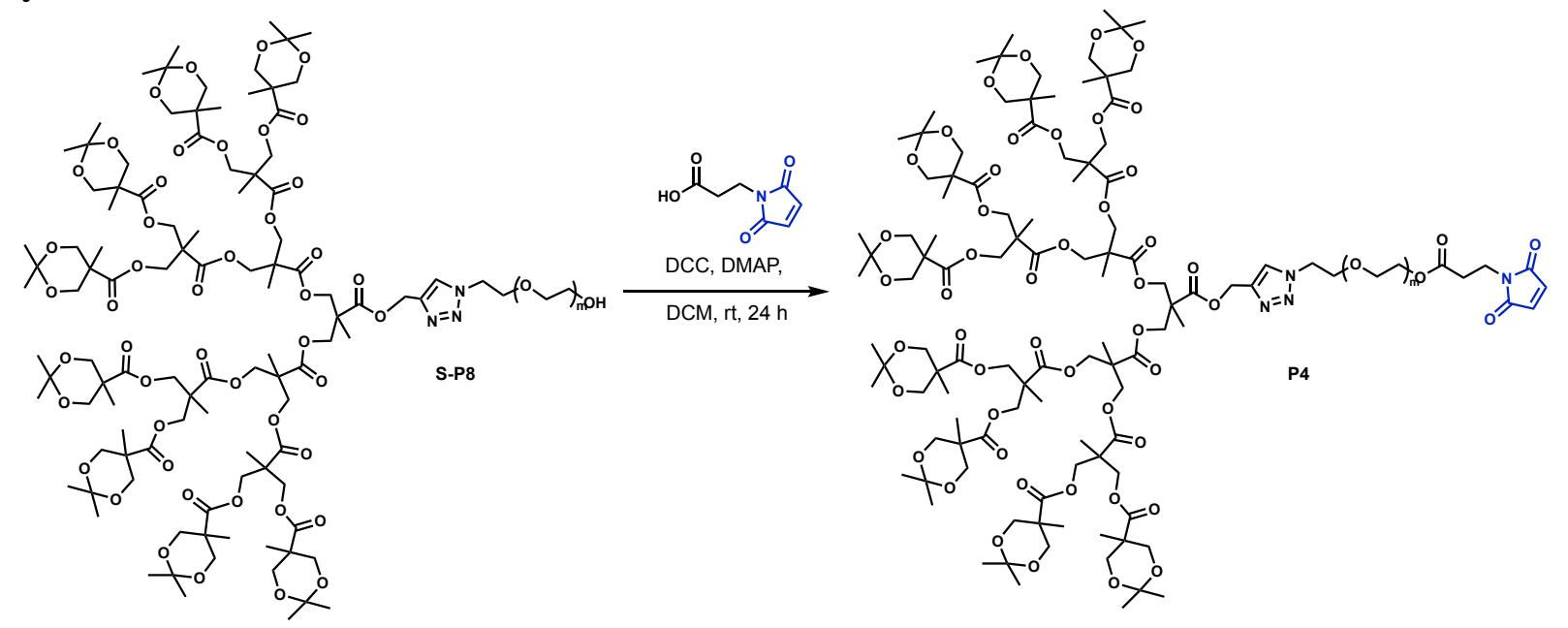

S-P8 (52.5 mg, $0.012 \mathrm{mmol}, 1$ eq.), 3-maleimidopropionic acid (8.1 mg, $0.048 \mathrm{mmol}, 4$ eq.), DCC (7.4 mg, $0.036 \mathrm{mmol}, 3$ eq.), DMAP (146 $\mu \mathrm{L}$ of $1 \mathrm{mg} / \mathrm{mL}$ stock solution in DCM, $0.001 \mathrm{mmol}, 0.1$ eq.) and $0.2 \mathrm{~mL}$ DCM was added to a $2-\mathrm{mL}$ vial equipped with stir bar. The vial was capped under air and allowed to stir for $24 \mathrm{~h}$. The solvent was removed, and the crude material was dialyzed against $650 \mathrm{~mL} \mathrm{MeOH} \mathrm{(1} \mathrm{kDa} \mathrm{MWCO} \mathrm{RC} \mathrm{tubing)} \mathrm{for} 16 \mathrm{~h}$ to afford $23 \mathrm{mg}(0.005 \mathrm{mmol}, 43 \%)$ of P4. $M_{\mathrm{n}(\mathrm{NMR})}=4,500 \mathrm{~g} / \mathrm{mol}, M_{\mathrm{n}(\mathrm{SEC})}=6,300 \mathrm{~g} / \mathrm{mol}, Ð=1.06$.

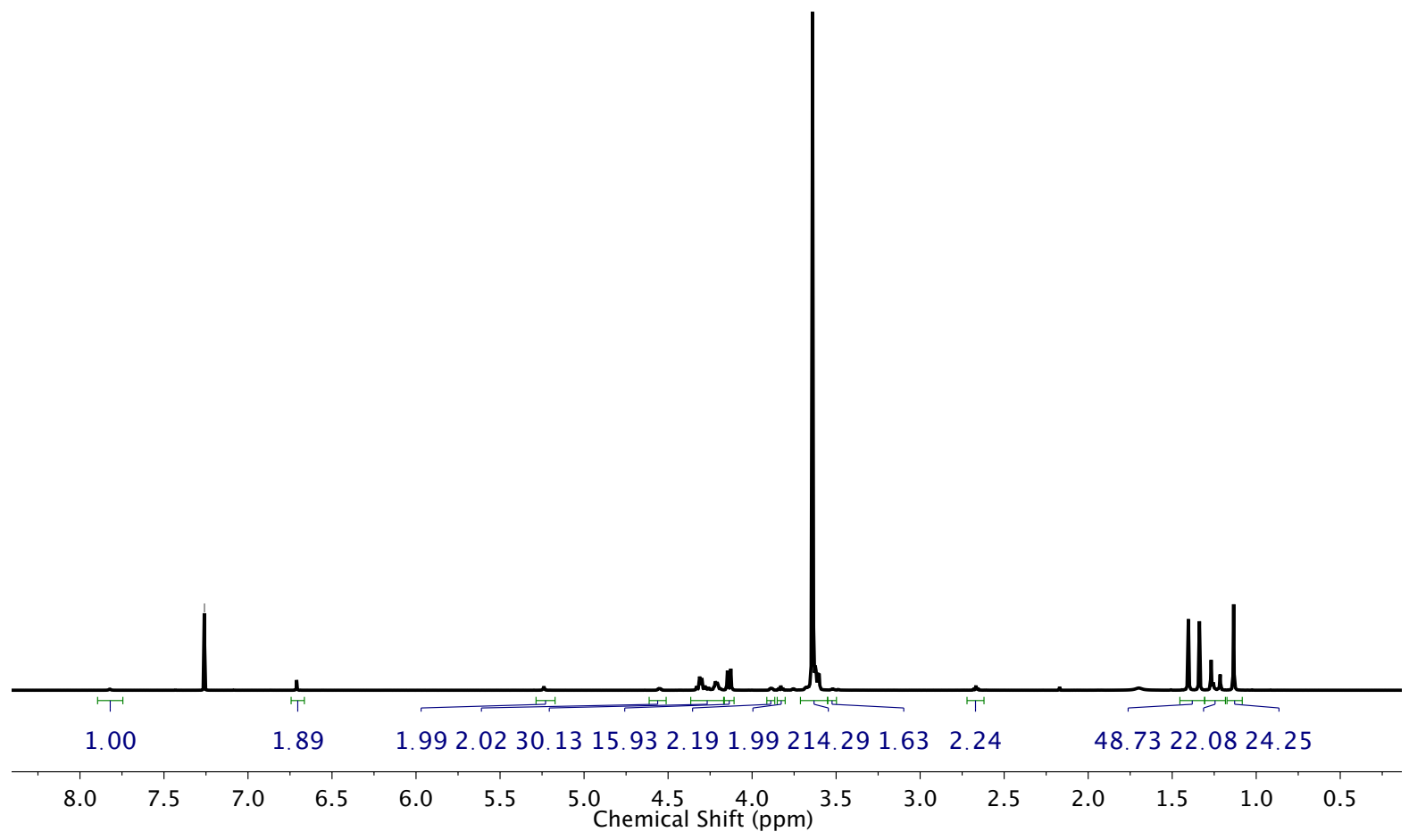

Figure S44. ${ }^{1} \mathrm{H}$ NMR of spectrum of $\mathbf{P 4}$ in $\mathrm{CDCl}_{3}$ showing presence of expected chain end resonance corresponding to maleimide after functionalization. 


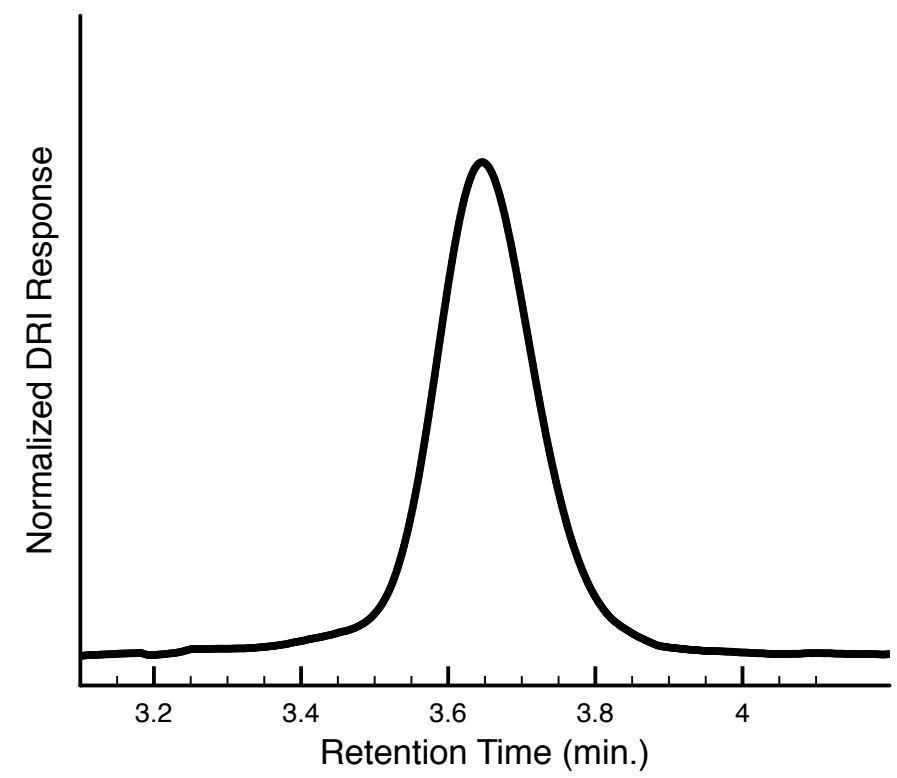

Figure S45. SEC-RI trace of P4.

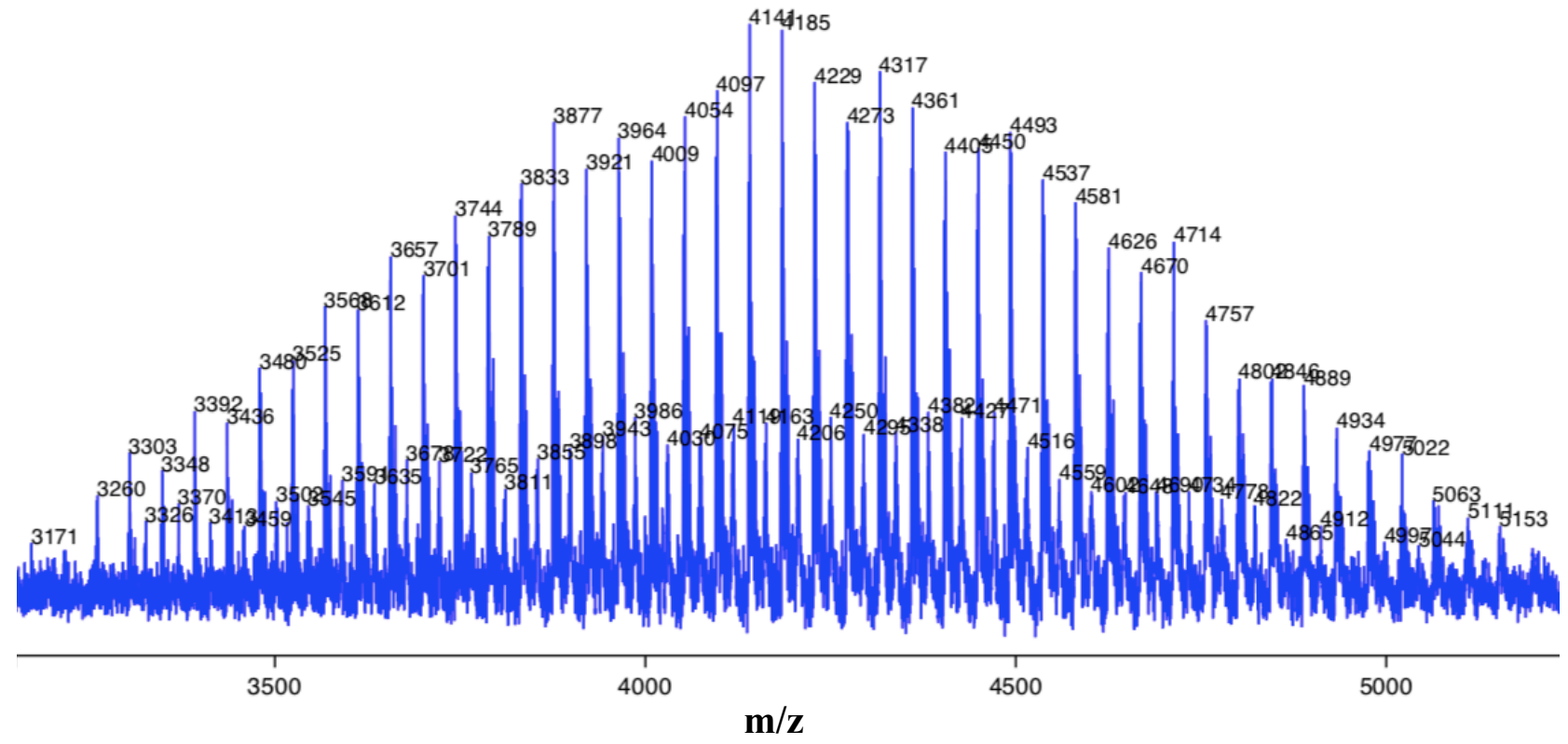

Figure S46. MALDI shows expected distribution of masses for $\mathbf{P 4}$. Calculated $[\mathrm{DP}=30+\mathrm{Na}]^{+}=$ 3743 , observed $=3744$. A second distribution is observed corresponding to additional coupling of second maleimide to unprotected dendron: Calculated $[\mathrm{DP}=28+\mathrm{Na}]^{+}=3766$, observed 3765 . 


\section{Synthesis of P5:}

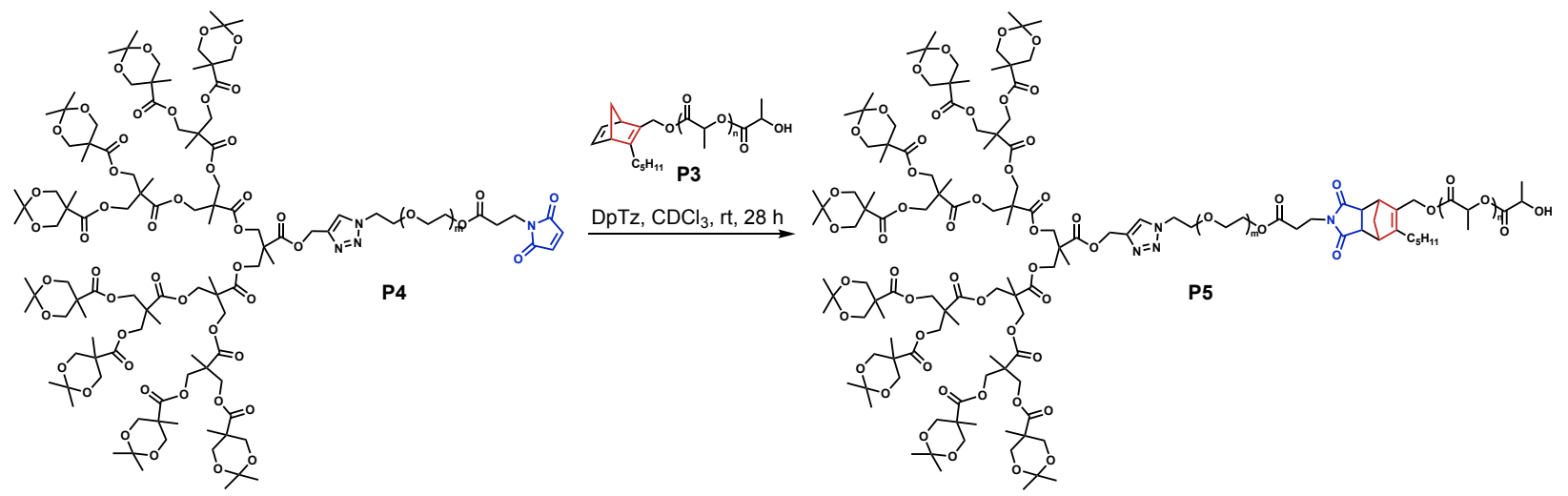

P3 (22.3 mg, $0.0011 \mathrm{mmol}, 1$ eq.), 3,6-di-2-pyridyl-1,2,4,5-tetrazine (DpTz) (0.5 mg, 0.0021 mmol, 1.9 eq.), and $\mathbf{P 4}$ ( $7.8 \mathrm{mg}, 0.0017 \mathrm{mmol}, 1.5$ eq.) were added to a $2 \mathrm{~mL}$ vial, and dissolved in $0.3 \mathrm{~mL}$ of $\mathrm{CDCl}_{3}$. The vial was capped under air and stirred at $\mathrm{rt}$ for $28 \mathrm{~h}$. The excess DpTz was quenched with approximately $0.2 \mathrm{mg}$ norbornene and stirred an additional $30 \mathrm{~min}$. The solvent was removed, and the crude polymer precipitated twice in $10 \mathrm{~mL}$ cold $\mathrm{MeOH}\left(-78{ }^{\circ} \mathrm{C}\right)$ to afford 8 $\mathrm{mg}(29 \%)$ of P5. $M_{\mathrm{n}(\mathrm{SEC})}=28,100 \mathrm{~g} / \mathrm{mol}, \emptyset=1.04$.

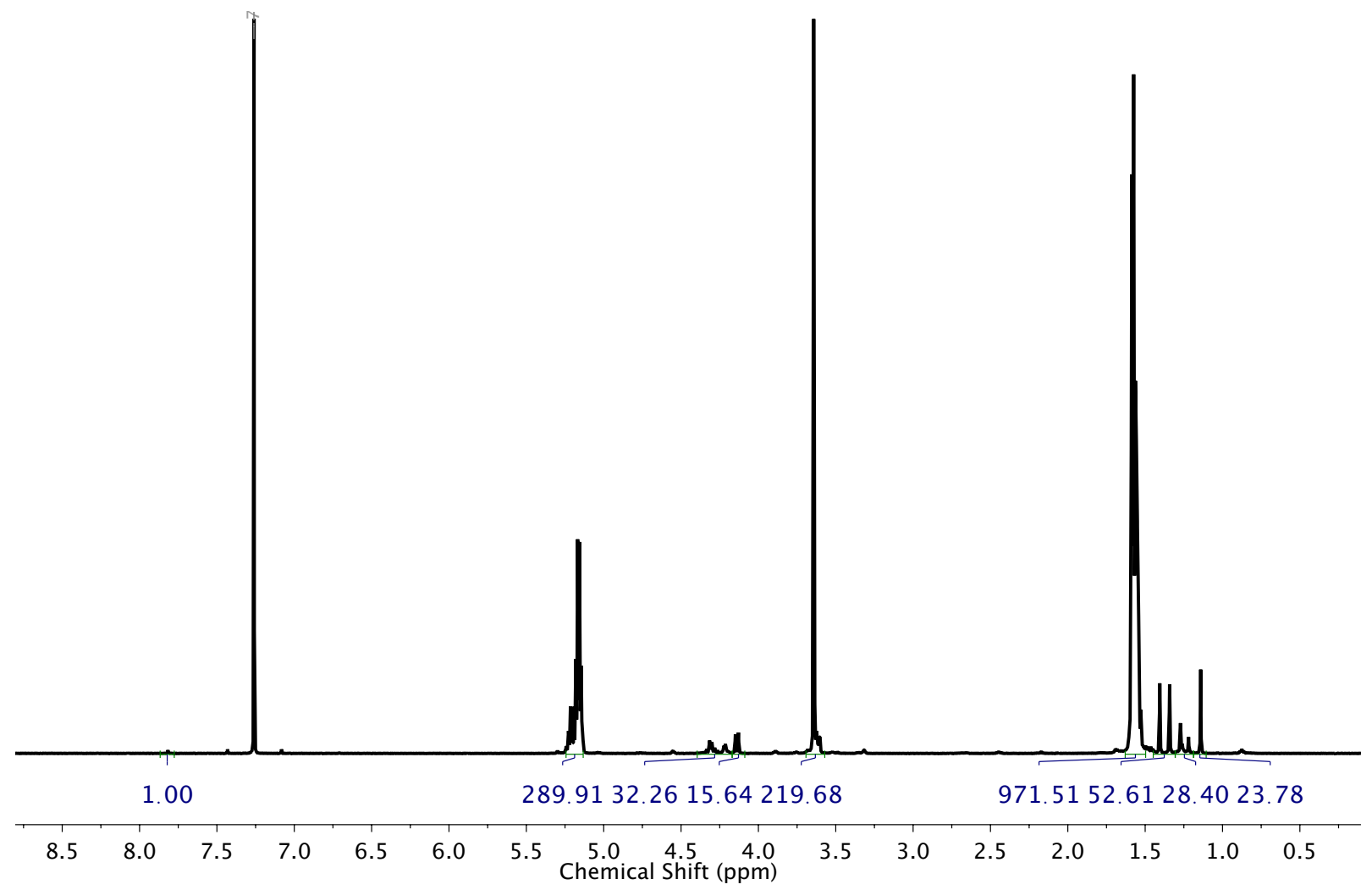

Figure S47. ${ }^{1} \mathrm{H}$ NMR of spectrum of $\mathbf{P 5}$ in $\mathrm{CDCl}_{3}$ showing presence of triazole, dendron, PEG and PLA resonances. 


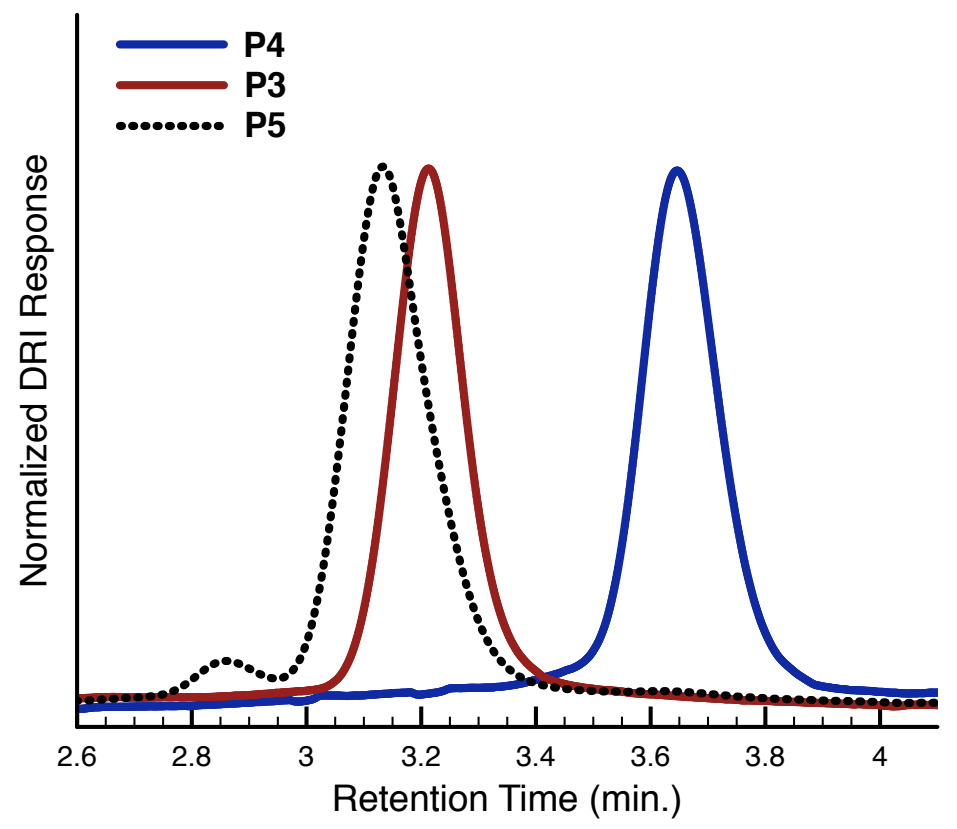

Figure S48. Overlay of SEC-RI traces of P3, P4 and P5 showing expected shift to higher molecular weight after NBD click. Higher molecular weight peak attributed to double click of P3 to bifunctional material observed in MALDI of P4 (see Figure S46).

\section{Synthesis of P6:}

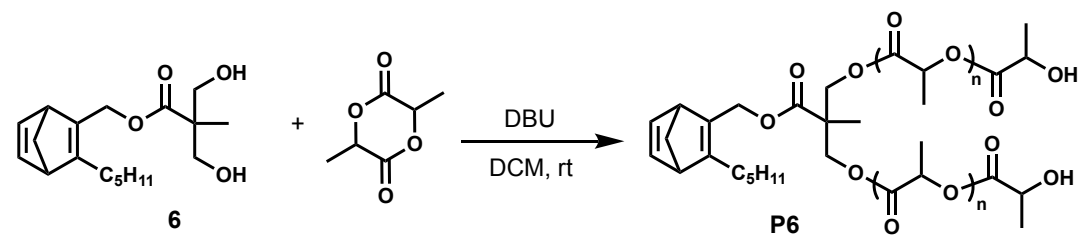

A 20-mL scintillation vial equipped with a magnetic stir bar was flame dried under argon and vacuum cycles for a total of 3 cycles, before transferring into a glovebox. To the vial, $D, L$-lactide $(2.1 \mathrm{~g}, 14.5 \mathrm{mmol}, 150 \mathrm{eq}$.) was added and dissolved in $10 \mathrm{~mL}$ of anhydrous DCM. Following this, compound 6 ( $29.9 \mathrm{mg}, 0.097 \mathrm{mmol}, 1$ eq.) was added along with a THF solution of DBU (1.5 M, $65 \mu \mathrm{L}, 0.097 \mathrm{mmol}, 1$ eq.). The polymerization was allowed to react at $\mathrm{rt}$ for $8 \mathrm{~min}$ and quenched with benzoic acid (59.0 mg, $0.48 \mathrm{mmol}, 5$ eq.). Monomer conversion was estimated by ${ }^{1} \mathrm{H}$ NMR in deuterated chloroform and determined to be $76 \%$. The DCM was removed under reduced pressure and the crude mixture was re-dissolved in a minimal amount of DCM. The mixture was subsequently precipitated into cold methanol before collecting the solid via vacuum filtration. The solid was re-dissolved in DCM and the precipitation process was repeated twice. The obtained white solid was dried under reduced pressure. $M_{\mathrm{n} \text { (theoretical) }}=16,000 \mathrm{~g} / \mathrm{mol}, M_{\mathrm{n}(\mathrm{NMR})}=17,000$ $\mathrm{g} / \mathrm{mol}, M_{\mathrm{n}(\mathrm{SEC})}=23,800 \mathrm{~g} / \mathrm{mol}, \doteq=1.04$. 


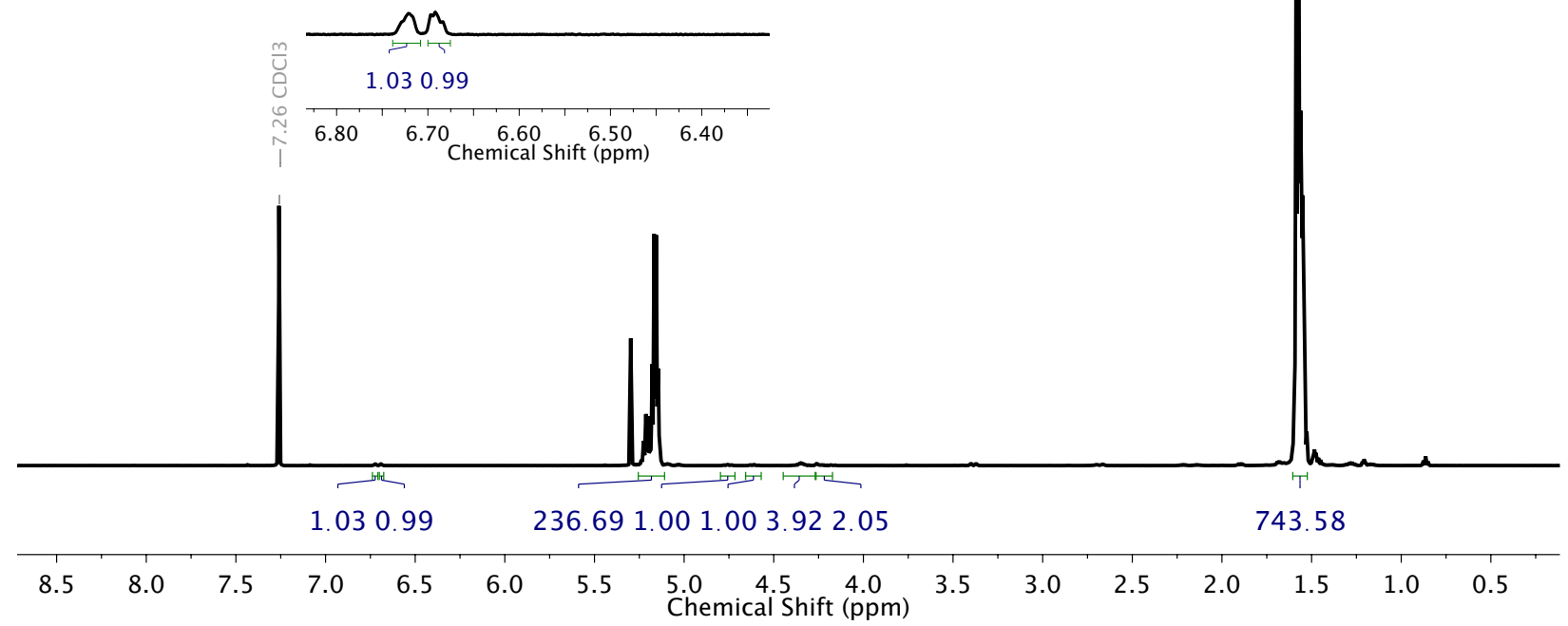

Figure S49. ${ }^{1} \mathrm{H}$ NMR of spectrum of $\mathbf{P 6}$ in $\mathrm{CDCl}_{3}$ showing presence of expected chain-end resonances.

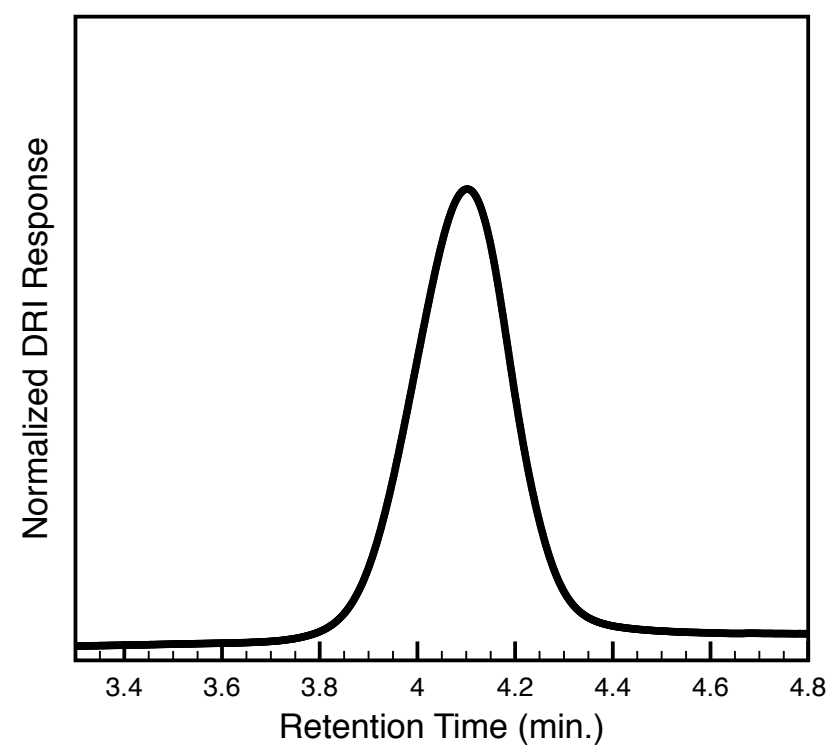

Figure S50. SEC-RI trace of P6. 


\section{Synthesis of P7:}
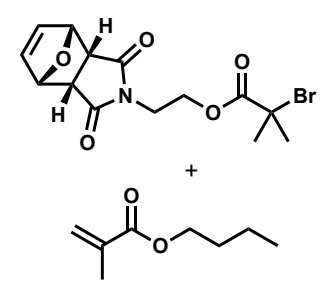
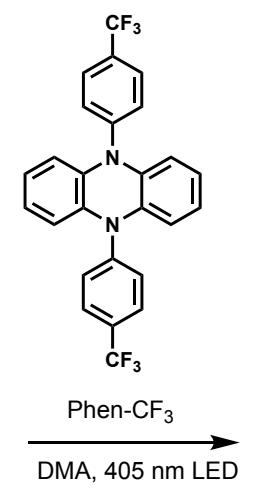
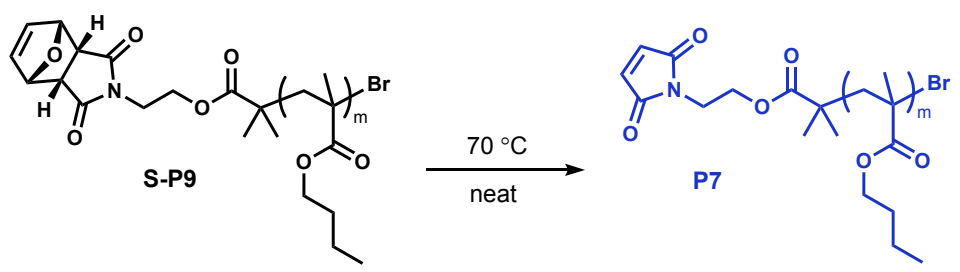

Adapted from literature procedure. ${ }^{5}$ Phen- $\mathrm{CF}_{3}$ photocatalyst $(1.5 \mathrm{mg}, 0.003 \mathrm{mmol}, 0.05$ eq.) was added to a $20 \mathrm{~mL}$ scintillation vial containing $1.2 \mathrm{~mL}$ of $N, N$-dimethylacetamide (DMA) and allowed to stir in the dark for $15 \mathrm{~min}$. At this time, $n$-butyl methacrylate ( $n$-BuMA) (1.2 mL, 7.56 mmol, 120 eq.) was added along with endo furan protected-maleimide ATRP initiator (22.6 mg, $0.06 \mathrm{mmol}, 1$ eq.). The reaction mixture was degassed by bubbling with argon for $10 \mathrm{~min}$ before wrapping the cap with parafilm. The vial was subsequently irradiated under a $405 \mathrm{~nm}$ collimated LED for $20 \mathrm{~h}$. Monomer conversion was estimated to be $58 \%$ based on analysis of the crude mixture by ${ }^{1} \mathrm{H}$ NMR. The crude polymerization mixture was precipitated once into hexanes and collected via vacuum filtration. The resulting solid was re-dissolved in a minimal amount of DCM and precipitated into cold methanol. Precipitation into methanol was repeated a total of 2 times to yield S-P9. Approximately $60 \mathrm{mg}$ of resulting polymer (S-P9) was subsequently heated neat under vacuum at $70{ }^{\circ} \mathrm{C}$ for $16 \mathrm{~h}$ to yield $\mathbf{P 7}$ in quantitative yield. Conversion $58 \%, M_{\text {n(theoretical }}=10,000$ $\mathrm{g} / \mathrm{mol}, M_{\mathrm{n}(\mathrm{SEC})}=9,600 \mathrm{~g} / \mathrm{mol}, \nexists=1.26$. 

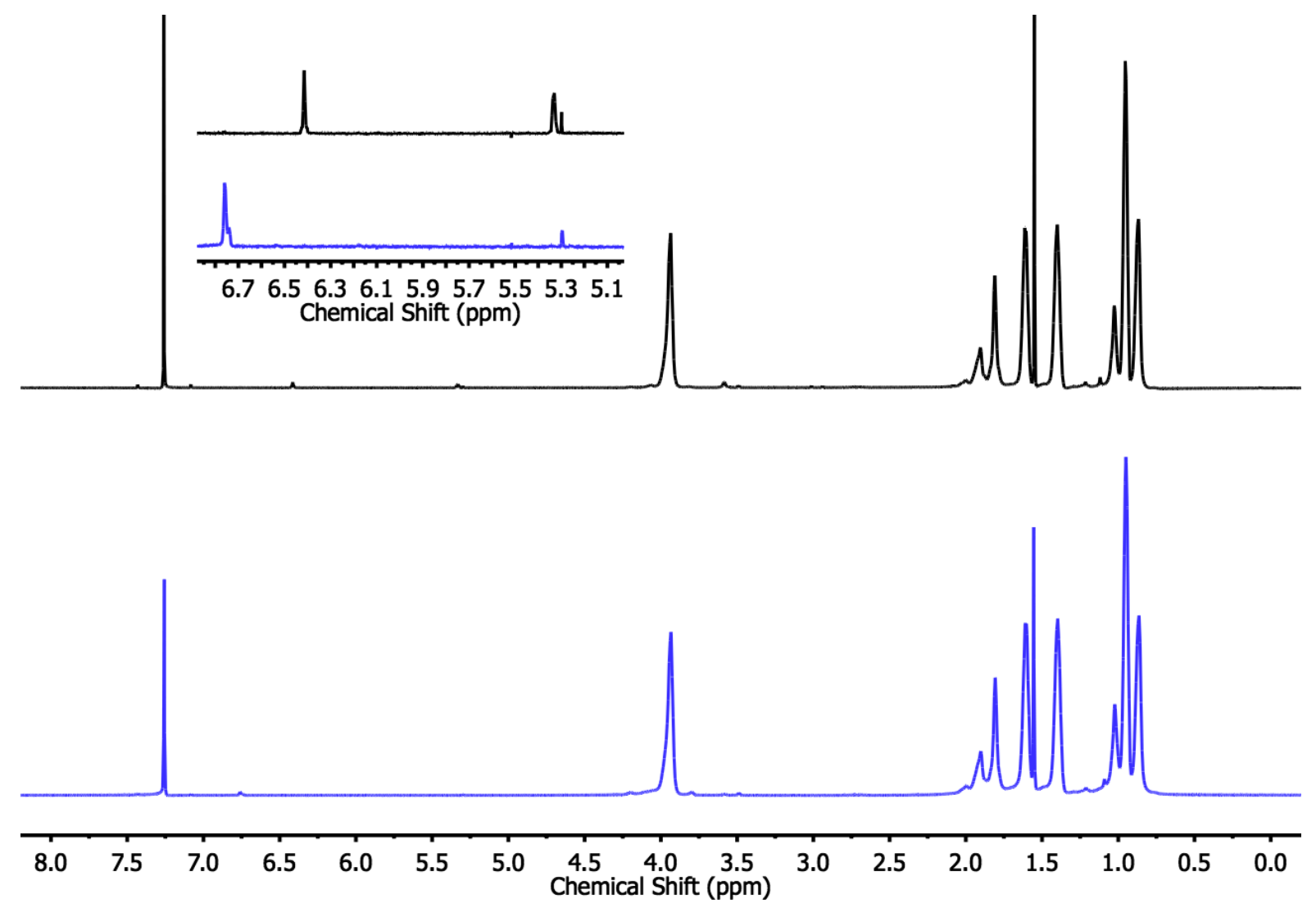

Figure S51. ${ }^{1} \mathrm{H}$ NMR of spectra overlay of $\mathbf{S - P 9}$ and $\mathbf{P 7}$ in $\mathrm{CDCl}_{3}$ confirming successful deprotection as following heating at $70^{\circ} \mathrm{C}$.

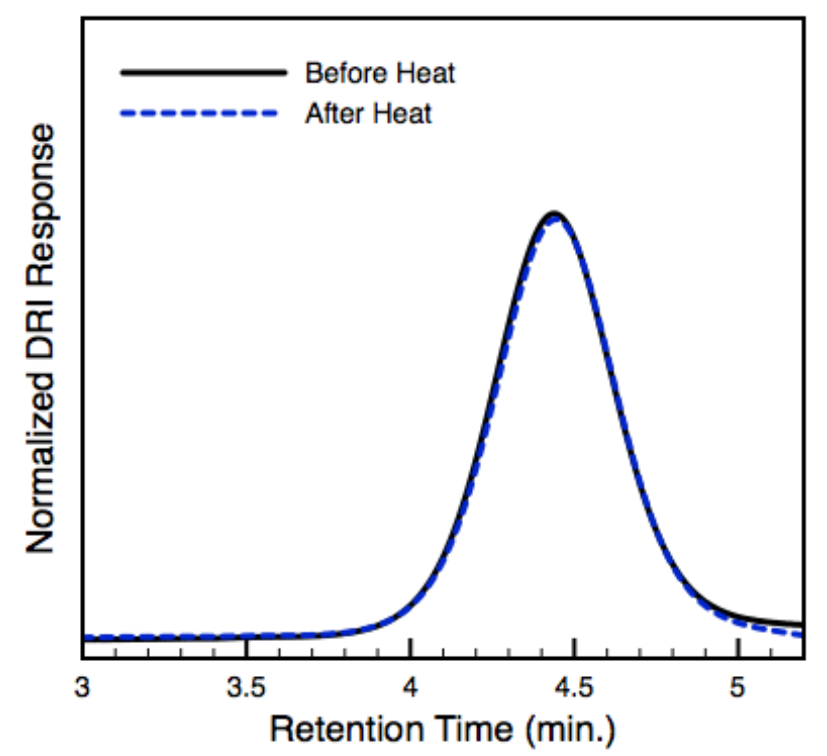

Figure S52. Overlay of SEC-RI traces of S-P9 and P7 before and after heating shows no observable change in molar mass distribution. 


\section{Synthesis of P8:}

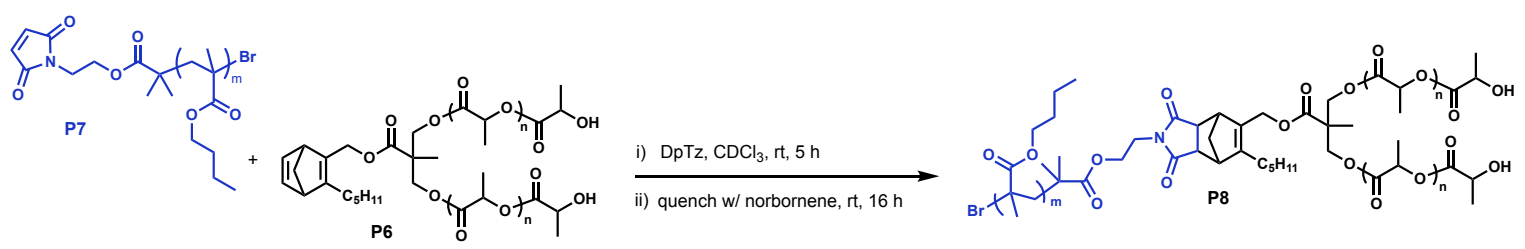

P6 (64 mg, 0.004 mmol, 1 eq.), 3,6-di-2-pyridyl-1,2,4,5-tetrazine (DpTz) (3.8 mg, 0.016 mmol, 4 eq.) and $\mathbf{P 7}$ (54 mg, $0.006 \mathrm{mmol}, 1.5$ eq.) were added to a 1-dram vial equipped with a septum cap and dissolved in $0.64 \mathrm{~mL}$ of $\mathrm{CDCl}_{3}$. The vial was placed on a shaker and allowed to react for $5 \mathrm{~h}$ at $\mathrm{rt}$ before adding approximately $3 \mathrm{mg}$ of norbornene to quench the remaining DpTz. The reaction was allowed to stir for an additional $16 \mathrm{~h}$. SEC analysis of the crude mixture showed expected shift in retention time and remaining P7. The crude reaction mixture was precipitated into 50:50 $\mathrm{MeOH}: \mathrm{IPA}$ and centrifuged to collect the desired product. The supernatant was discarded and the resulting solid was collected, and re-dissolved in a minimal amount of DCM before repeating the precipitation process. This process was repeated a total of 4 times. The resulting solid was collected and dried under reduced pressure before performing additional characterization using ${ }^{1} \mathrm{H} \mathrm{NMR}$, SEC and SAXS. $M_{\mathrm{n}(\mathrm{SEC})}=35,000 \mathrm{~g} / \mathrm{mol}, \emptyset=1.04$

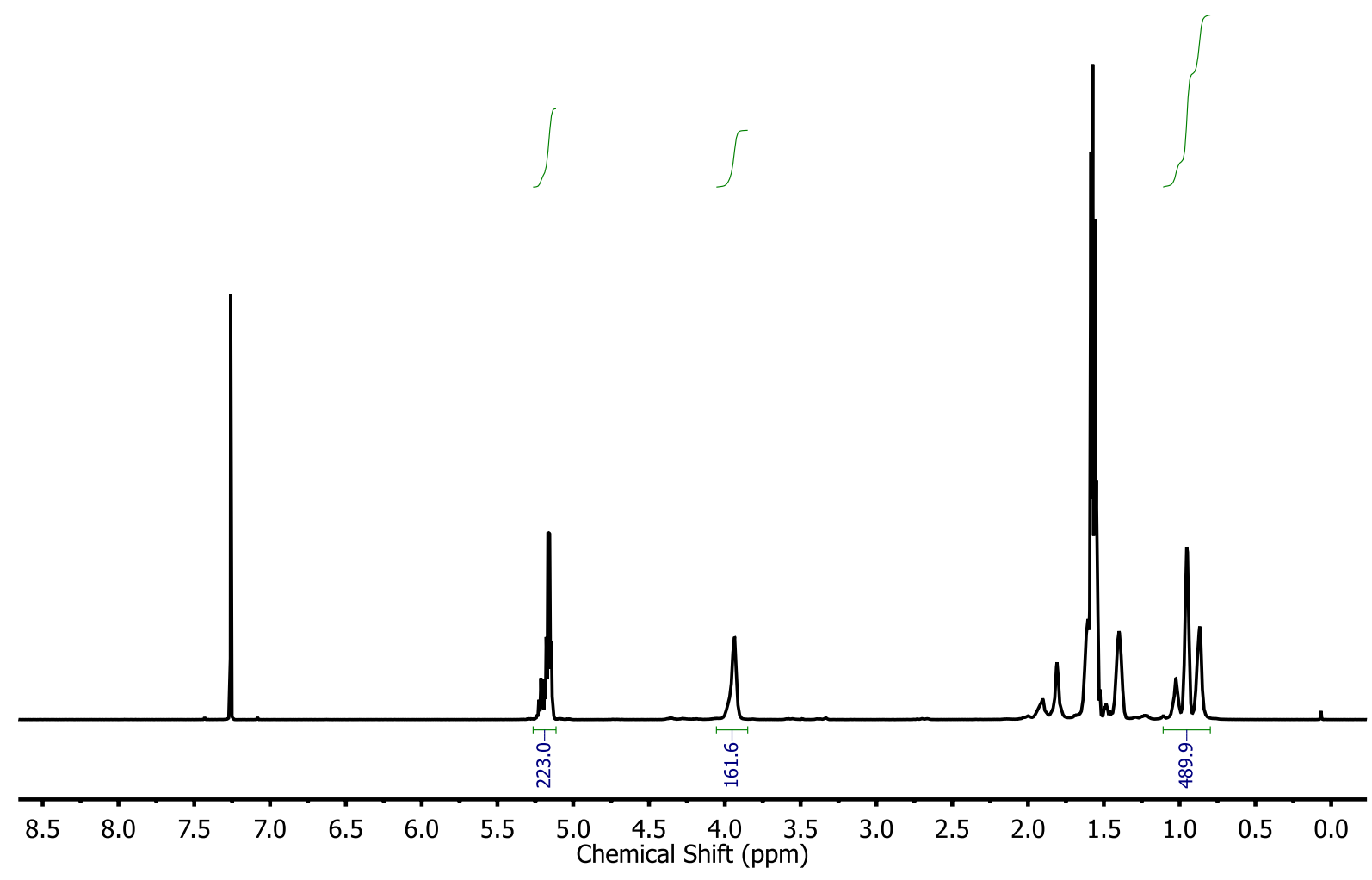

Figure S53. ${ }^{1} \mathrm{H}$ NMR of spectrum of purified $\mathbf{P 8}$ confirming presence of both blocks and a product block polymer composition of $f_{\mathrm{PLA}}=0.54$ based on reported homopolymer densities. ${ }^{6,7}$ 


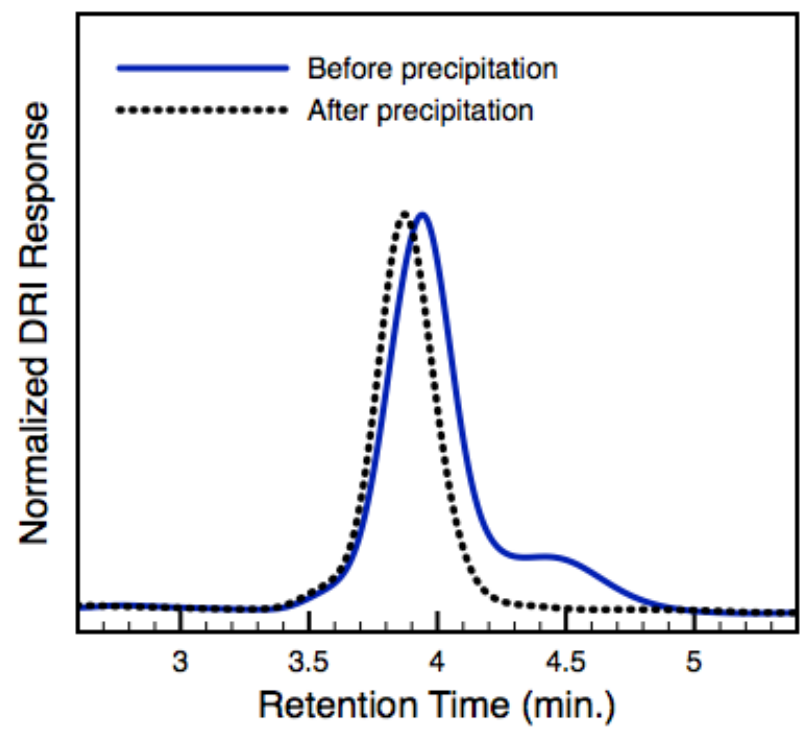

Figure S54. Overlay of SEC-RI traces of P8 before and after precipitation shows successful removal of slight excess of $\mathbf{P 8}$. 


\section{Synthesis and characterization of S-P12:}
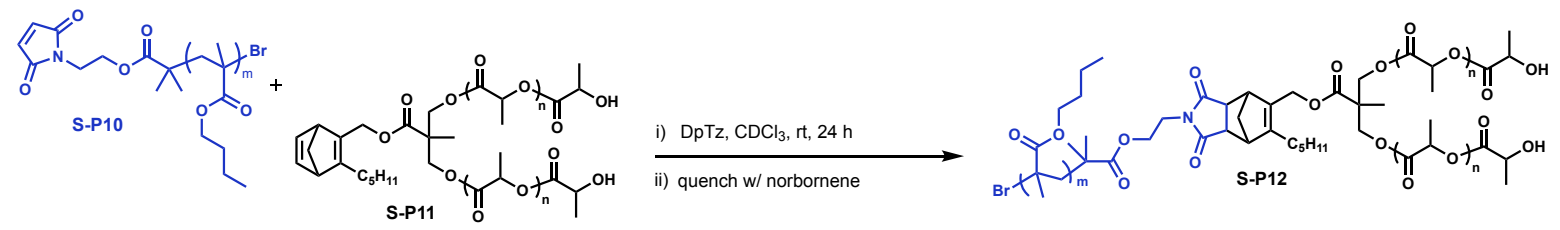

S-P10 (41 mg, $0.007 \mathrm{mmol}, 1.5$ eq.), S-P11 ( $81 \mathrm{mg}, 0.005 \mathrm{mmol}, 1.0$ eq.) and 3,6-di-2-pyridyl1,2,4,5-tetrazine (DpTz) (1.6 mg, $0.007 \mathrm{mmol}, 1.5$ eq.) were added to a 1-dram vial equipped with a septum cap and dissolved in $0.80 \mathrm{~mL}$ of $\mathrm{CDCl}_{3}$. The vial was placed on a shaker and allowed to react for $24 \mathrm{~h}$ at $\mathrm{rt}$ before adding approximately $2 \mathrm{mg}$ of norbornene to quench the remaining DpTz. The reaction was allowed to stir for an additional $30 \mathrm{~min}$. SEC analysis of the crude mixture showed expected shift in retention time and remaining S-P10. The crude reaction mixture was precipitated into 50:50 MeOH:IPA and centrifuged to collect the desired product. The supernatant was discarded and the resulting solid was collected, and re-dissolved in a minimal amount of DCM before repeating the precipitation process. This process was repeated a total of 4 times. The resulting solid was collected and dried under reduced pressure before performing additional characterization using ${ }^{1} \mathrm{H}$ NMR, SEC and SAXS. $M_{\mathrm{n}(\mathrm{S}-\mathrm{P} 10, \mathrm{SEC})}=6,000 \mathrm{~g} / \mathrm{mol}, \nexists=1.39, M_{\mathrm{n}(\mathrm{S}-\mathrm{P} 11 \text {, }}$ $\mathrm{SEC})=29,300 \mathrm{~g} / \mathrm{mol}, \nexists=1.04, M_{\mathrm{n}(\mathrm{S}-\mathrm{P} 12, \mathrm{SEC})}=36,400 \mathrm{~g} / \mathrm{mol}, \nexists=1.04$.

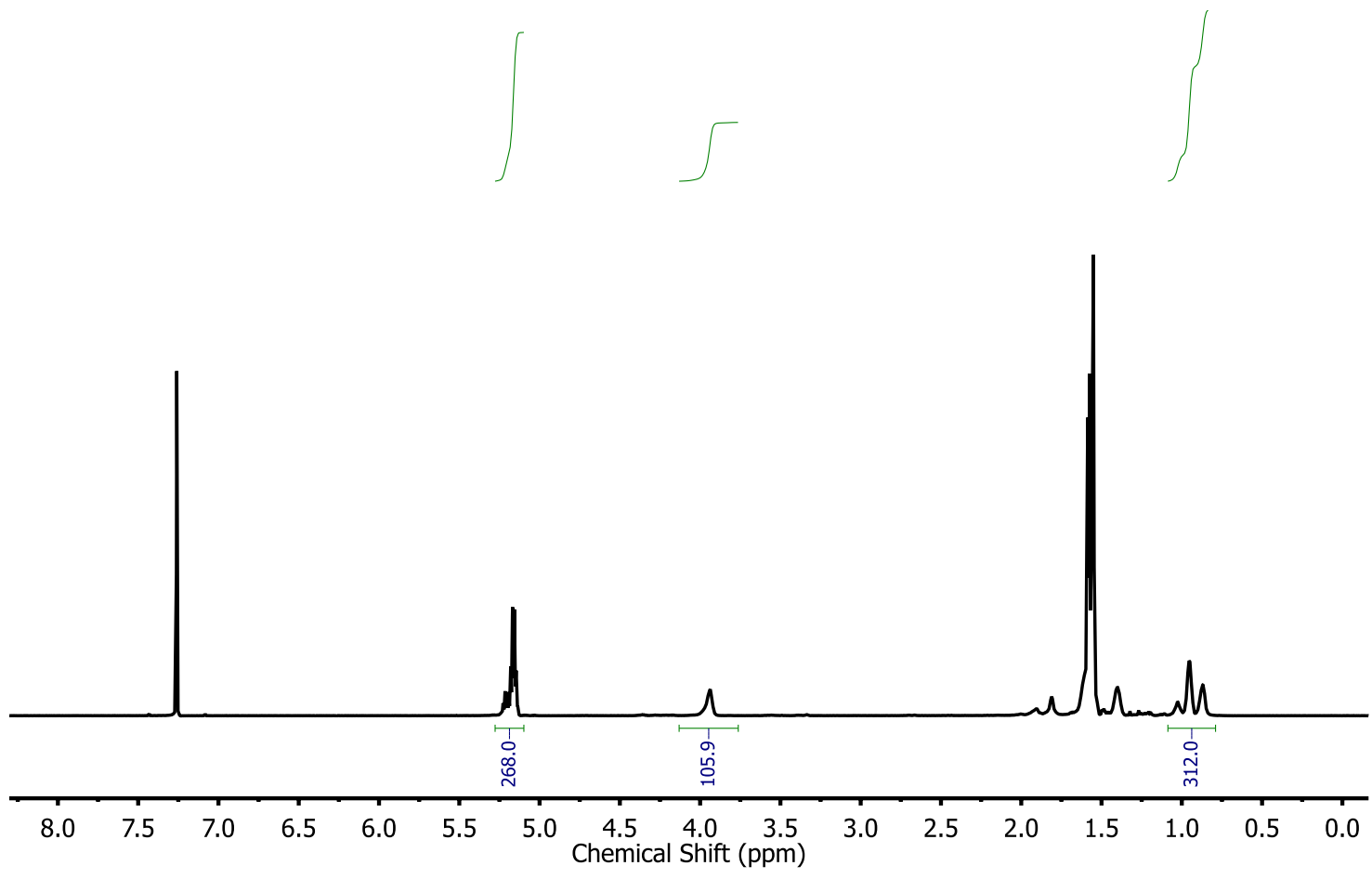

Figure S55. ${ }^{1} \mathrm{H}$ NMR spectrum of purified S-P12 confirming presence of both blocks and a product block polymer composition of $f_{\mathrm{PLA}}=0.68$ based on reported homopolymer densities. ${ }^{6,7}$ 


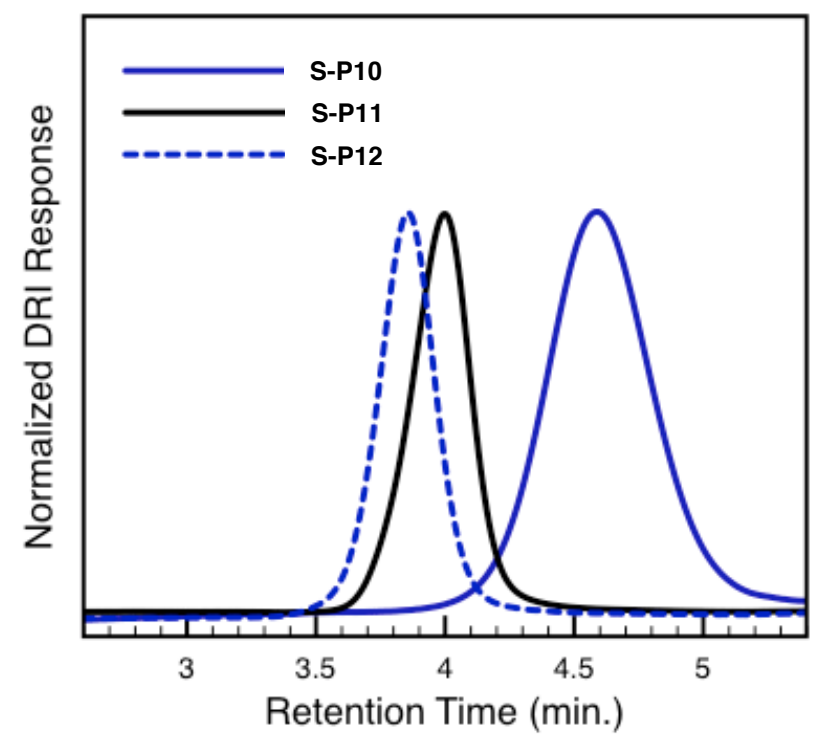

Figure S56. Overlay of SEC-RI traces of S-P10, S-P11 and S-P12 showing the expected shift to lower retention time indicative of a higher molar mass product.

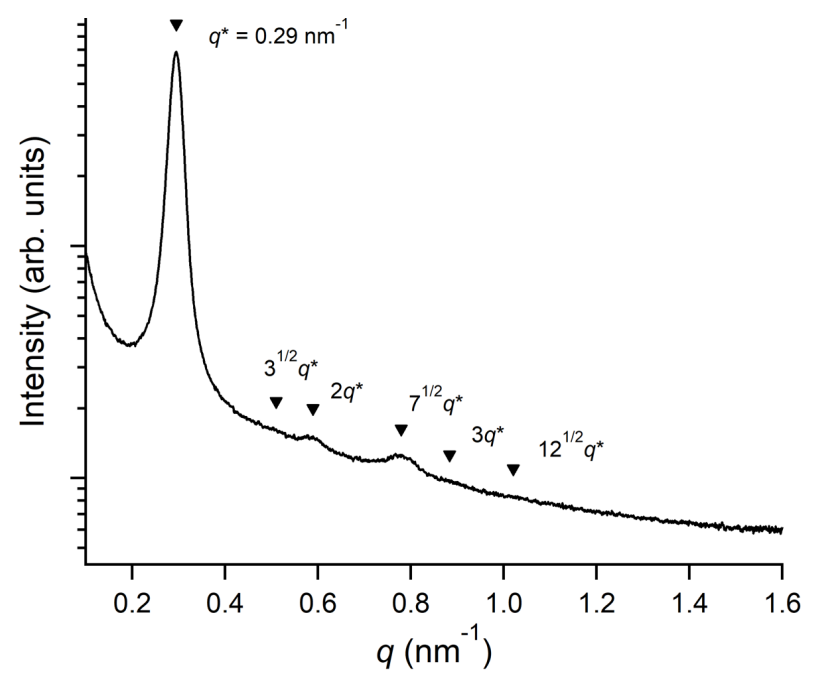

Figure S57. SAXS analysis of S-P12 corresponding to a hexagonally close-packed cylindrical morphology. 


\section{References}

(1) Tuktarov, A. R.; Akhmetov, A. R.; Khuzin, A. A.; Dzhemilev, U. M. Synthesis and Properties of Energy-Rich Methanofullerenes Containing Norbornadiene and Quadricyclane Moieties. J. Org. Chem. 2018, 83 (7), 4160-4166.

(2) Jishkariani, D.; MacDermaid, C. M.; Timsina, Y. N.; Grama, S.; Gillani, S. S.; Divar, M.; Yadavalli, S. S.; Moussodia, R.-O.; Leowanawat, P.; Berrios Camacho, A. M.; et al. SelfInterrupted Synthesis of Sterically Hindered Aliphatic Polyamide Dendrimers. Proc. Natl. Acad. Sci. 2017, 114 (12), E2275 LP-E2284.

(3) Hedrick, J. L.; Trollsås, M.; Hawker, C. J.; Atthoff, B.; Claesson, H.; Heise, A.; Miller, R. D.; Mecerreyes, D.; Jérôme, R.; Dubois, P. Dendrimer-like Star Block and Amphiphilic Copolymers by Combination of Ring Opening and Atom Transfer Radical Polymerization. Macromolecules 1998, 31 (25), 8691-8705.

(4) Zhu, L.; Zhang, D.; Qu, D.; Wang, Q.; Ma, X.; Tian, H. Dual-Controllable Stepwise Supramolecular Interconversions. Chem. Commun. 2010.

(5) Discekici, E. H.; St. Amant, A. H.; Nguyen, S. N.; Lee, I. H.; Hawker, C. J.; Read De Alaniz, J. Endo and Exo Diels-Alder Adducts: Temperature-Tunable Building Blocks for Selective Chemical Functionalization. J. Am. Chem. Soc. 2018, 140 (15), 5009-5013.

(6) Orwoll, R. A. Densities, Coefficients of Thermal Expansion, and Compressibilities of Amorphous Polymers. In Physical Properties of Polymers Handbook; Mark, J. E., Ed.; Springer New York: New York, NY, 2007; pp 93-101.

(7) Mohanty, A. K.; Misra, M.; Drzal, L. T. Natural Fibers, Biopolymers, and Biocomposites; CRC press, 2005. 